Linköping University Medical Dissertations No. 1601.

\title{
Molecular Mechanisms of Reward and Aversion
}

\author{
Anna Mathia Klawonn
}

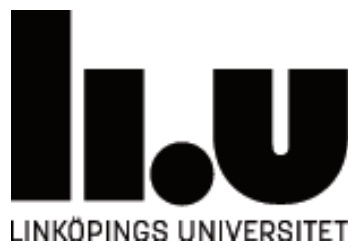

Department of Clinical and Experimental Medicine Linköping University, Sweden Linköping 2017 
Cover: Confocal microscopy image of D1R-tdTomato (red) and MC4R-eGFP (green) in the Striatum.

Published articles and figures have been reprinted with permission of the copyright holders.

(C)Anna M. Klawonn, 2017

Printed in Sweden by LiU-Tryck, Linköping, Sweden, 2017.

ISBN: 978-91-7685-412-9

ISSN: 0345-0082 


\section{Til mine kære, Michael og Mor}

The darker the night, the brighter the stars...

Dostojevskij 


\section{LIST OF STUDIES}

This thesis is based on the following papers, which will be referred to in the text by their Roman numerals.

I. Fritz $M^{*}$, Klawonn AM*, Nilsson A, Singh A, Zajdel J, Wilhelms DB, Lazarus M., Löfberg A, Jaarola M, Kugelberg UÖ, Billiar TR, Hackam DJ, Sodhi CP, Breyer MD, Jakobsson J, Schwaninger M, Schütz G, Parkitna JR, Saper CB, Blomqvist A, Engblom D. 2016. Prostaglandin-dependent modulation of dopaminergic neurotransmission elicits inflammation-induced aversion in mice. The Journal of Clinical Investigation 126(2): 695-705.

II. Fritz M*, Klawonn AM*, Jaarola M, Engblom D. 2018. Interferon-y mediated signaling in the brain endothelium is critical for inflammationinduced aversion. Brain, Behavior, and Immunity 67: 54-58.

III. Klawonn AM*, Fritz M*, Nilsson A, Shionoya K, Mirrasekhian E, Karlsson U, Jaarola M, Granseth B, Blomqvist A, Michaelides M, Engblom D. 2017. Motivational Valence is determined by Striatal Melanocortin 4 receptors. Manuscript in revision.

IV. Klawonn AM, Björk-Wilhelms D, Lindström SH, Singh AK, Jaarola M, Wess Jürgen, Fritz M, Engblom D. 2017. Muscarinic M4 receptors on Cholinergic and D1R-expressing neurons have opposing functionality for positive reinforcement and influence impulsivity. Manuscript in submission.

*These Authors contributed equally to this work. 


\section{ABSTRACT}

Various molecular pathways in the brain shape our understanding of good and bad, as well as our motivation to seek and avoid such stimuli. This work evolves around how systemic inflammation causes aversion; and why general unpleasant states such as sickness, stress, pain and nausea are encoded by our brain as undesirable; and contrary to these questions, how drugs of abuse can subjugate the motivational neurocircuitry of the brain. A common feature of these various disease states is involvement of the motivational neurocircuitry - from mesolimbic to striatonigral pathways. Having an intact motivational system is what helps us evade negative outcomes and approach natural positive reinforcers, which is essential for our survival. During disease-states the motivational neurocircuitry may be overthrown by the molecular mechanisms that originally were meant to aid us.

In study I, to investigate how inflammation is perceived as aversive, we used a behavioral test based on Pavlovian place conditioning with the aversive inflammatory stimulus E. coli lipopolysaccharide (LPS). Using a combination of cell-type specific gene deletions, pharmacology, and chemogenetics, we uncovered that systemic inflammation triggered aversion by MyD88-dependent activation of the brain endothelium followed by COX1-mediated cerebral prostaglandin E2 (PGE2) synthesis. Moreover, we showed that inflammation-induced PGE2 targeted EP1 receptors on striatal dopamine D1 receptor-expressing neurons and that this signaling sequence induced aversion through GABA-mediated inhibition of dopaminergic cells. Finally, inflammation-induced aversion was not an indirect consequence of fever or anorexia but constituted an independent inflammatory symptom triggered by a unique molecular mechanism. Collectively, these findings demonstrate that PGE2-mediated modulation of the dopaminergic circuitry is a key mechanism underlying inflammation-induced aversion.

In study II, we investigate the role of peripheral IFN- $\gamma$ in LPS induced conditioned place aversion by employing a strategy based on global and cell-type specific gene deletions, combined with measures of gene-expression. LPS induced IFN- $\gamma$ expression in the blood, and deletion of IFN-y or its receptor prevented conditioned place aversion (CPA) to LPS. LPS increased the expression of chemokine Cxc110 in the striatum of normal mice. This induction was absent in mice lacking IFN- $\mathrm{y}$ receptors or Myd88 in blood brain barrier endothelial cells. Furthermore, inflammation-induced aversion was blocked in mice lacking Cxcl10 or its receptor Cxcr3. Finally, mice with a selective deletion of the IFN- $y$ receptor in brain endothelial cells did not develop inflammation-induced aversion. Collectively, these 
findings demonstrate that circulating IFN-y binding to receptors on brain endothelial cells which induces Cxcl10, is a central link in the signaling chain eliciting inflammation-induced aversion.

In study III, we explored the role of melanocortin 4 receptors (MC4Rs) in aversive processing using genetically modified mice in CPA to various stimuli. In normal mice, robust aversions were induced by systemic inflammation, nausea, pain and kappa opioid receptor-induced dysphoria. In sharp contrast, mice lacking MC4Rs displayed preference towards most of the aversive stimuli, but were indifferent to pain. The unusual flip from aversion to reward in mice lacking MC4Rs was dopamine-dependent and associated with a change from decreased to increased activity of the dopamine system. The responses to aversive stimuli were normalized when MC4Rs were re-expressed on dopamine D1 receptor-expressing cells or in the striatum of mice otherwise lacking MC4Rs. Furthermore, activation of arcuate nucleus proopiomelanocortin neurons projecting to the ventral striatum increased the activity of striatal neurons in a MC4R-dependent manner and elicited aversion. Our findings demonstrate that melanocortin signaling through striatal MC4Rs is critical for assigning negative motivational valence to harmful stimuli.

The neurotransmitter acetylcholine has been implied in reward learning and drug addiction. However, the role of cholinergic receptor subtypes in such processes remains elusive. In study IV we investigated the function of muscarinic M4Rs on dopamine D1R expressing neurons and acetylcholinergic neurons, using transgenic mice in various reward-enforced behaviors and in a "waiting"-impulsivity test. Mice lacking M4-receptors from D1-receptor expressing neurons exhibited an escalated reward seeking phenotype towards cocaine and natural reward, in Pavlovian conditioning and an operant self-administration task, respectively. In addition, the M4-D1RCre mice showed impaired waiting impulsivity in the 5-choice-serialreaction-time-task. On the contrary, mice without M4Rs in acetylcholinergic neurons were unable to learn positive reinforcement to natural reward and cocaine, in an operant runway paradigm and in Pavlovian conditioning. Immediate early gene expression mirrored the behavioral findings arising from M4R-D1R knockout, as cocaine induced cFos and FosB was significantly increased in the forebrain of M4D1RCre mice, whereas it remained normal in the M4R-ChatCre mice. Our study illustrates that muscarinic M4Rs on specific neural populations, either cholinergic or D1R-expressing, are pivotal for learning processes related to both natural reward and drugs of abuse, with opposing functionality. 


\section{POPULÄRVETENSKAPLIG SAMMANFATTNING}

\section{Molekylära mekanismer bakom belöning och aversion}

Olika signalkretsar i hjärnan formar vår förståelse av gott och dåligt. Dessa kretsar är aktiva under all vår vakna tid, genom hela livet. De gör att vi uppsöker saker som är bra för oss, såsom mat och trevligt sällskap, medan vi undviker saker som är skadliga eller gör oss sjuka. Dessa kretsar är därför nödvändiga för vår överlevnad. De hjärnområden som är ansvariga för värdering och motivation till att söka det goda och undvika det dåliga är mitthjärnan som innehåller dopamin nervceller, vilka leder till vårt belöningscentrum i framhjärnan. Vid sjukdomstillstånd påverkas funktionen i dopamin belöningskretsen och de molekylära mekanismer som i friskt tillstånd är fördelaktiga för vår överlevnad kan vid sjukdom orsaka omfattande lidande.

I min avhandling har vi identifierat flera molekylära mekanismer som reglerar det dopaminerga belöningssystemet vid olika sjukdomstillstånd. Vi har studerat hur inflammation, smärta och stress orsakar nedstämdhet och aversion, men också hur specifika molekylära förändringar i belöningssystemet kan leda till utveckling av drogberoende. Genom att studera dessa kretsar i prekliniska djurmodeller, hoppas vi att kunna utveckla behandlingar mot sjukdomar som betvingar vårt humör.

I min första studie hittade vi orsaken till varför inflammatorisk sjukdom leder till olustkänslor och nedstämdhet. Vid sjukdomar som ger upphov till inflammation, som till exempel infektioner, frisätter immunceller inflammatoriska ämnen i blodet. I en musmodell för bakteriell infektion upptäckte vi att de inflammatoriska ämnena i blodet aktiverar blodkärl i hjärnan, vilket leder till bildning av ett hormonliknande ämne, prostaglandin E2. Prostaglandin E2 binder sedan till specifika receptorer på nervceller i belöningssystemet och minskar dopamin nervcellernas aktivitet. Den sänkta aktiviteten i belöningssystemet leder till olustkänslor och aversion.

I min andra studie identifierade vi ett annat signalämne i blodet som också orsakar aversion. Detta ämne, som heter interferon gamma, är viktigt för signaleringen från immuncellerna till hjärnans kärl.

I min tredje studie blev vi förvånade! När vi studerade möss som saknade en specifik receptor i hjärnan upptäckte vi att dessa möss föredrog saker som gjorde dom sjuka. De betedde sig som om de tyckte om inflammation lika mycket som vanliga möss gillar kokain. Djuren som saknade denna så kallade melanokortin 4 receptor tycktes även uppskatta saker som gjorde dem stressade eller illamående. Vi såg att djuren utan melanokortin-receptorn hade ett inverterat dopaminsvar på sjukdomsrelaterade 
stimuli. I den normala hjärnan sjunker dopaminhalterna vid inflammation, medans i hjärnor utan melanokortin-receptorn ökar dopaminnivån specifikt i belöningscentret.

Vi upptäckte att aktivering av de nervceller som frisätter signalämnet som binder till melanokortin 4 receptorn leder till aversion. Signalämnet gör detta genom att aktivera melanokortin 4 receptorn $i$ en specifik grupp av nervceller $i$ belöningssystemet. Detta är den första studie som visar att en specifik receptor på en särskild typ av nervceller kan omkasta vår uppfattning om vad som är bra och dåligt. Våra resultat visar hur tätt belöning och aversion är knutna med varandra i hjärnan.

Slutligen fann vi att en typ av acetylkolinreceptorer (M4) är ansvariga för att dämpa utvecklingen av beroenderelaterade beteenden. Då vi tog bort dessa receptorer från hjärnans belöningssystem i möss, blev mössen mer impulsiva och mer intresserade av att söka efter kokain än normalt. Djuren utan M4-receptorn i belöningssystemet visade också ökad tendens till återfall, vilket är relevant eftersom det är mycket svårt att förebygga återfall till beroende med de nuvarande behandlingarna. Våra resultat kan hjälpa till att förklara varför olikheter i gensekvensen för M4 receptorn påverkar risken för beroende i människa. 


\section{TABLE OF CONTENTS}

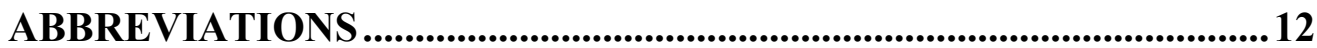

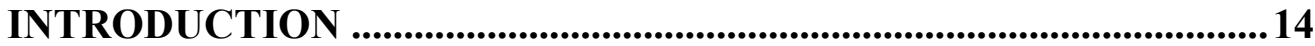

NEUROBIOLOGY OF MOTIVATION ......................................................... 14

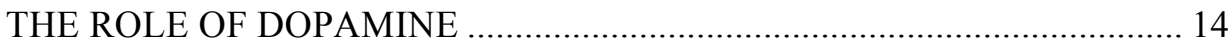

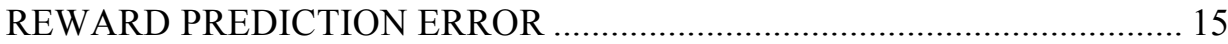

THE STRIATAL DIRECT AND INDIRECT PATHWAYS............................ 17

SYSTEMIC INFLAMMATION AND AFFECTIVE STATE …….................. 18

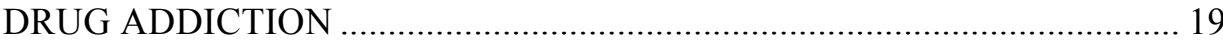

Reward allostasis ......................................................................... 20

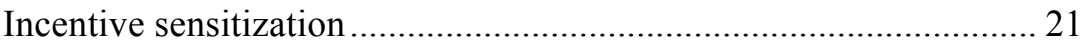

Dopaminergic circuitry and striatal pathways................................... 22

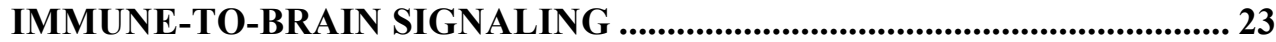

CYTOKINES IN MOTIVATION AND AFFECTIVE STATE ....................... 23

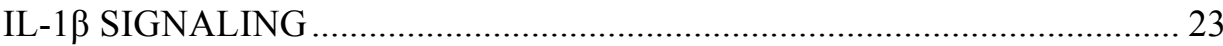

Myd88 and Toll-like receptors...................................................... 24

IL-1 $\beta$ in motivation and affective state ........................................... 24

TNF $\alpha$-SIGNALING IN MOTIVATION AND AFFECTIVE STATE .............25

IFN $\gamma$-SIGNALING IN MOTIVATION AND AFFECTIVE STATE ..............27

SIGNALING ACROSS THE BLOOD-BRAIN-BARRIER ………............... 28

PROSTAGLANDIN E2 SYNTHESIS AND RECEPTORS .............................30

MELANOCORTINS IN MOTIVATION AND AFFECTIVE STATE ......... 32

THE ARCUATE NUCLEUS MELANOCORTIN CIRCUITRY .................... 32

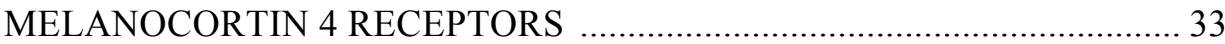

ACETYLCHOLINE IN MOTIVATION AND ADDICTION ...........................35

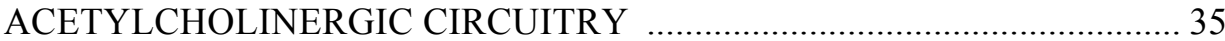

ACETYLCHOLINE AND REINFORCEMENT LEARNING .......................35

THE MUSCARINIC ACETYLCHOLINE RECEPTOR M4 ........................... 36

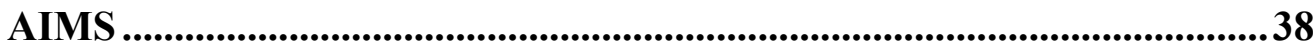

METHODOLOGICAL CONSIDERATIONS........................................39

EXPERIMENTAL ANIMALS - THE MOUSE ………................................. 39

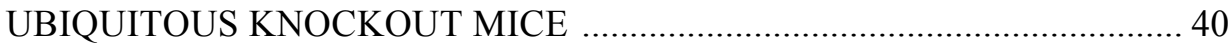

CONDITIONAL STRATEGIES FOR KNOCKOUT AND KNOCKIN ........41

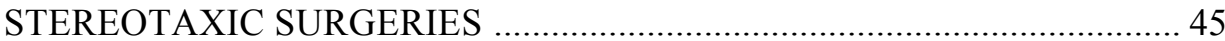

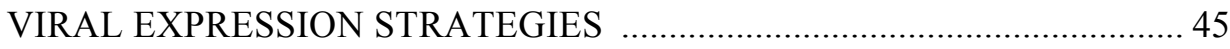

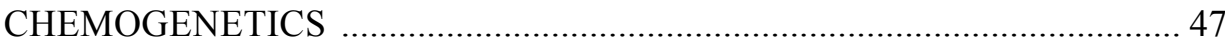




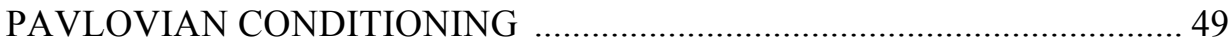

MODELS OF SYSTEMIC INFLAMMATION ……........................................ 51

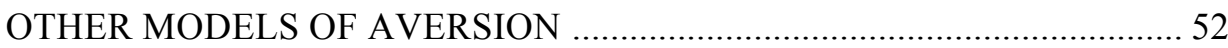

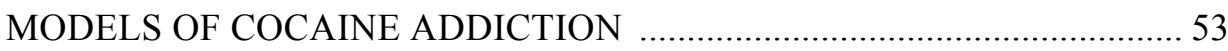

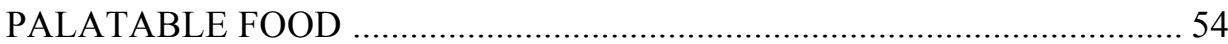

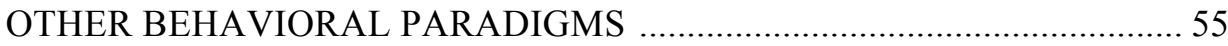

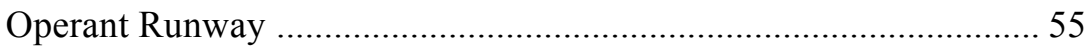

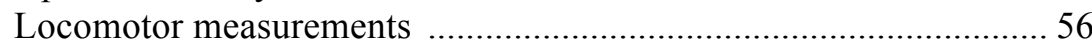

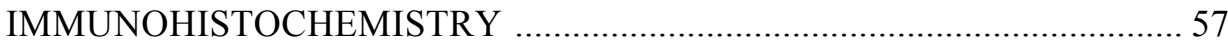

ENZYME-LINKED IMMUNOSORBENT ASSAYS …..............................58

ELECTROPHYSIOLOGY AND OPTOGENETICS …...................................59

RESULTS AND DISCUSSION

STUDY I. PROSTAGLANDIN-DEPENDENT MODULATION OF

DOPAMINERGIC NEUROTRANSMISSION ELICITS INFLAMMATION-

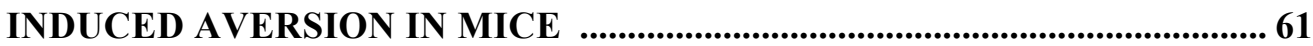

CYTOKINE AND TOLL-LIKE RECEPTOR SIGNALING ACROSS THE

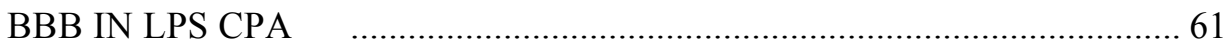

PGE2 SIGNALING IN THE DORSAL STRIATUM VIA D1R NEURONS

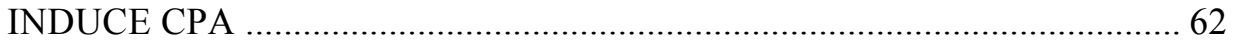

INFLAMMATION LEADS TO DECREASED DOPAMINE MEDIATING

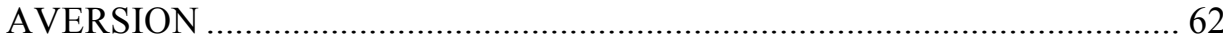

STUDY I. DISCUSSION ..............................................................................63

INFLAMMATION INDUCED CONDITIONED PLACE AVERSION - A

NEW WAY OF MONITORING INFLAMMATION INDUCED NEGATIVE

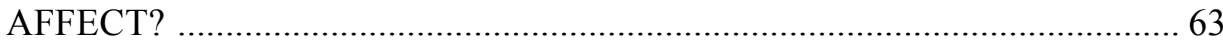

IMMUNE-TO-BRAIN SIGNALING ACROSS THE BBB - THE MISSING

LINK TO CPU PGE2-SYNTHESIS AND THE MISSING CELL ................. 65

PGE2 SIGNALING TO MOTIVATIONAL NEUROCIRCUITRY - TIME

FOR A REVISION OF THE ROLE OF NIGROSTRIATAL CIRCUITS IN

MOTIVATION?

STUDY II. INTERFERON- $\gamma$ MEDIATED SIGNALING IN THE BRAIN ENDOTHELIUM IS CRITICAL FOR INFLAMMATION-INDUCED

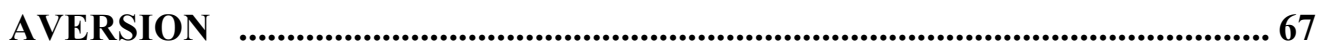

STUDY II. DISCUSSION ......................................................................68

CYTOKINES SIGNAL ACROSS THE BBB ENDOTHELIUM VIA MYD88

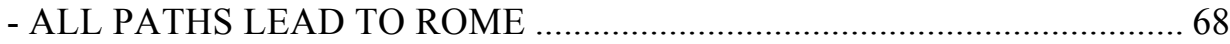

INTERFERONS AND CXCL10 IN NEGATIVE AFFECT - A NEW

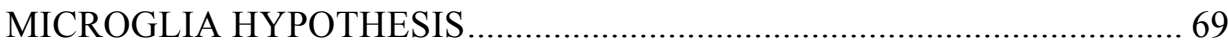


STUDY III. MOTIVATIONAL VALENCE IS DETERMINED BY STRIATAL

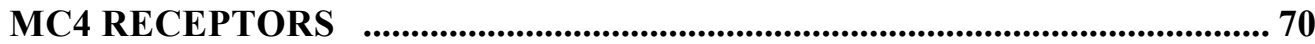

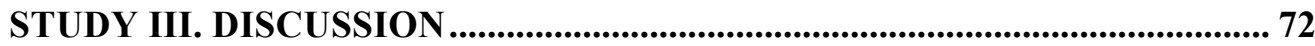

STUDY IV. MUSCARINIC M4 RECEPTORS ON CHOLINERGIC AND D1REXPRESSING NEURONS HAVE OPPOSING FUNCTIONALITY FOR POSITIVE REINFORCEMENT AND INFLUENCE IMPULSIVITY ............76

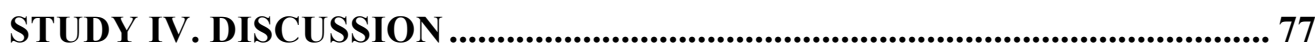
M4R FUNCTION IN LEARNING AND MOTIVATION - CAN WE REALLY TELL? 78

CONCLUDING REMARKS AND PERSPECTIVES .............................80 IMMUNE-TO-BRAIN SIGNALING - UNIVERSAL PRINCIPLES AND

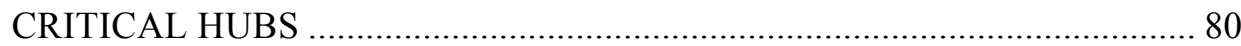
DOPAMINERGIC NEURONS IN MOTIVATION AND NEGATIVE AFFECT - CONTROVERSIES AND NEW PERSPECTIVES 81

ACKNOWLEDGEMENTS 83

REFERENCES 86 


\section{ABBREVIATIONS}

$\alpha-\mathrm{MSH}$

AAVs

AgRP

ARC

BBB

BNST

ChAT

Chr2

$\mathrm{CNO}$

CNS

COX $1 / 2$

$\mathrm{CPA}$

CPP

$\mathrm{CPu}$

CS

CVOs

Cxcl10/IP10

D1R

D2R

DAT

DIO

DREADDs

E. coli

ELISA

ERK

Floxed

GABA

GIRK

GPCR

GPe

HPA-axis

6-OH-DA

hSyn

i.c.v

i.p.

i.v.

IDO

IFN- $\gamma$

IL-1R

IL-1RAP

IL-1 $\beta$
Melanocyte stimulation hormone

Adeno associated viruses

Agouti-related peptide

Arcuate nucleus of the hypothalamus

Blood-brain barrier

Bed nucleus stria terminalis

Choline acetyltransferase

Channelrhodopsin

Clozapine-N-oxide

Central nervous system

Cyclooxygenase $1 / 2$

Conditioned place aversion

Conditioned place preference

Caudate putamen/dorsal striatum

Conditioned stimulus

Circumventricular organs

Interferon- $\gamma$ Protein 10

Dopamine D1-receptors

Dopamine D2-receptors

Dopamine transporter

Double-floxed inverted open reading frame

Designer receptors exclusively activated by designer drugs

Escherichia coli

Enzyme-linked Immunosorbent Assay

Extracellular signal-regulated kinase

Flanked by lox-P sites

Gamma-aminobutyric acid

G-protein coupled inward rectifying potassium channel

G-protein coupled receptor

Globus pallidus external

Hypothalamic-pituitary-adrenal axis

6-hydroxydopamine

Human synapsin 1 gene promoter

Intracerebroventricular

Intraperitoneal

Intravenous

Indoleamine-2,3-deoxygenase

Interferon- $\gamma$

IL-1 receptor

IL-1 receptor accessory protein

Interleukin-1- $\beta$ 


$\begin{array}{ll}\text { IL-6 } & \text { Interleukin } 6 \\ \text { IL-1Ra } & \text { IL-1 receptor specific antagonist } \\ \text { IRAK1/4 } & \text { IL-1 receptor-associated kinase 1/4 } \\ \text { JAK } & \text { Janus activated kinase } \\ \text { JNK c-Jun } & \text { N-terminal kinase } \\ \text { KO } & \text { Knockout } \\ \text { KOR } & \text { Kappa-opioid receptor } \\ \text { LDT } & \text { The laterodorsal tegmental nucleus } \\ \text { LiCl } & \text { Lithium Chloride } \\ \text { M4R } & \text { Muscarinic acetylcholine receptor M4 } \\ \text { MAPK } & \text { Mitogen-activated protein kinase } \\ \text { MC4R } & \text { Melanocortin 4 receptor } \\ \text { MCP-1 } & \text { Chemoattractant protein-1 } \\ \text { mPFC } & \text { Medial prefrontal cortex } \\ \text { mPGES1 } & \text { Microsomal PGE2 synthase } \\ \text { MSN } & \text { Medium spiny neuron } \\ \text { MyD88 } & \text { Myeloid differentiation factor } 88 \\ \text { NAc } & \text { Nucleus Accumbens } \\ \text { NFKB } & \text { Nuclear factor } \text { kB } \\ \text { NMDA } & \text { N-methyl-D-aspartate } \\ \text { NTS } & \text { Nucleus of the solitary tract } \\ \text { PAMPs } & \text { Pathogen-associated molecular patterns } \\ \text { PGE2 } & \text { Prostaglandin E2 } \\ \text { PKC } & \text { Protein kinase C } \\ \text { POMC } & \text { Proopiomelanocortin } \\ \text { PPT } & \text { Pedunculopontine nucleus } \\ \text { PRRs } & \text { Pattern-recognition receptors } \\ \text { ptger4 } & \text { PGE2 EP4-receptor gene } \\ \text { PVH } & \text { Paraventricular hypothalamic nucleus } \\ \text { s.c. } & \text { Subcutaneous } \\ \text { SN } & \text { Substantia nigra pars compacta } \\ \text { SSRIs } & \text { Selective serotonin reuptake inhibitors } \\ \text { sTNF } \alpha & \text { solubleTNF } \alpha \\ \text { TLRs } & \text { Toll-like receptors } \\ \text { TNF } \alpha & \text { Tumor-necrosis-factor- } \alpha \\ \text { TNF } \alpha \text { R1/2 } & \text { TNF } \alpha \text { receptor type 1 and 2 } \\ \text { US } & \text { Unconditioned stimulus } \\ \text { VP } & \\ \text { VTA } & \\ & \text { Ventral pallidum } \\ & \end{array}$




\section{INTRODUCTION}

The general aim of this doctoral thesis is to investigate how specific molecular pathways shape our understanding of good and bad, as well as our motivation to seek and avoid such stimuli. This work evolves around how systemic inflammation causes negative affect; and why general unpleasant states such as sickness, stress, pain and nausea are encoded by our brain as undesirable; and contrary to these questions, how drugs of abuse can subjugate the motivational neurocircuitry of the brain. Having an intact motivational system is what helps us evade negative outcomes and approach natural positive reinforcers, which is essential for our survival. During disease-states this motivational neurocircuitry may be overthrown by the molecular mechanisms that originally were meant to aid us.

\section{NEUROBIOLOGY OF MOTIVATION}

\section{The role of Dopamine}

Our motivational system is primarily the mesolimbic pathway, which is composed of the dopaminergic neurons in the ventral tegmental area (VTA) projecting to the nucleus accumbens (NAc). The motivational literature to a large extend ignores the nigro-striatal dopamine system, i.e. substantia nigra pars compacta (SN) dopaminergic projections to dorsal striatum $(\mathrm{CPu})$. Yet, Wolfram Shultz's first findings on the important learning principle "reward prediction error", described in the next section, were primarily based on recordings from SN dopamine-neurons (Shultz and Romo, 1990; Shultz et al., 1997). Furthermore, the SN-CPu connectivity has been shown to be involved in both positive and negative motivational states (Belin and Everitt, 2007; Ilango et al., 2014). Irrespective of their location, dopamine neurons exhibit two specific firing patters: A tonic, steady-state firing (ca. $4 \mathrm{~Hz}$ ) and a phasic, burst-firing $(>15 \mathrm{~Hz})$. Tonic firing reflects synaptic baseline-levels of dopamine, whereas phasic firing can be measured as synaptic peaks in dopaminelevels typically associated with reward signaling (Bromberg-Martin et al., 2010). The first discovery that dopamine plays a role in the perception of reward, was done in Stockholm by Urban Ungerstedt. He conducted experiments lesioning the dopamine fibers that traverse the hypothalamus using 6-hydroxydopamine (6-OH-DA), and observed a detrimental impact on food-intake and other behaviors (Ungerstedt, 1971). Following the studies of Ungerstedt, investigations on the role of dopamine in reinforcement-related behaviors expanded numerously and led to the general idea that dopamine is responsible for signaling reward. In the 1990's Berridge, Robinson and Salamone challenged the concept of "reward" being a single psychological process, and the idea that such a process is intrinsically associated with the hedonic 
features of the reinforcing stimulus. To prove this concept, they did experiments lesioning dopamine neurons projecting to the NAc or $\mathrm{CPu}$ using 6-OH-DA in rats. In Salamone's experiments, normal rats would first learn a reward task, in which they were given the choice between an easy accessible small food-reward and a larger food-reward that would require them to climb a wall. Normal rats were motivated to climb the wall for the larger reward, but after lesioning dopamine neurons projecting to NAc the rats decreased their willingness to perform the task (Salamone et al., 1994). This suggested that dopamine was playing an essential role in reward evaluation and motivation. Berridge and Robinson further demonstrated that similar lesions of dopamine-neurons projecting to either NAc or $\mathrm{CPu}$ did not impact hedonic responses to sucrose (rhythmic tongue protrusions and paw licks) (Berridge and Robinson, 1998). These findings clarified that reward is not a unitary process, but rather a constellation of separate psychological phenomena. This led to the conceptual separation of motivation from pleasure, and illustrated that the role of dopamine primarily is signaling "incentive salience". In this manner dopamine is responsible for attributing positive significance to rewarding events and stimuli, and help motivate us to pursue these.

\section{Reward prediction error}

Wolfram Shultz conducted the first experiments demonstrating how dopamine neurons functionally signal reward evaluation. When submitting monkeys to a go/nogo ${ }^{1}$ reward task, he found that dopamine neurons respond to visual and auditory cues predicting the arrival of a reward by burst-firing (Shultz and Romo, 1990). In followup studies, he and others elucidated that dopamine neurons encode a reward prediction error, by which they teach us to predict outcomes of specific situations irrespective of valence (Shultz, 2013). The principle is simple: At the arrival of an unexpected reward dopamine neurons will burst-fire, leading to an increase of dopamine in downstream structures such as the striatum. With time and repeated exposure to this reward, the environment that predicts the arrival of the reward will instead lead to dopamine firing prior to the arrival of the reward. This makes sense as under natural circumstances rewards are retrieved after exposure to specific predictive cues (e.g. smells and visual and tactile cues). In this manner, the dopamine-signal has been transferred to the cues predicting the arrival of the reward. Essentially, we form associations with cues, which in turn will drive motivational

\footnotetext{
${ }^{1}$ In this task the monkeys would be placed in a small room in front of a wall with doors and hold one hand on a touch-sensitive key. At a trigger-stimulus (sound + opening of a door to a food-box) the monkey let go of the key to reach out and open the door of the food-box to receive a food-reward (GO) or remain motionless, holding the key for longer time, when another food-box door (NO-GO) would open to receive the food-reward.
} 
acts, and need dopamine to do so. Furthermore, this signal is adaptive; in the case that a reward is larger than anticipated, the dopamine neurons will increase their firing at arrival of the positive stimulus, which in turn will be transferred to the cuedopamine-signal. This will shape the cue-dopamine-signal to better predict outcomes in the future. On the other hand, if the reward following the predictive cue is smaller or completely absent, the dopamine-neurons will decrease firing, and thereby the synaptic baseline dopamine-levels will drop. With time and habit, through association formation, dopamine will predict the difference between a reward anticipated to occur and the final reward received (Shultz et al., 1997). Finally, the reward prediction error is bi-directional. In the case of aversive events, dopamine neurons will decrease their tonic firing, causing a pause in dopamine-input to downstream structures, and this decrease will with repeated exposure be transferred to the predictive cues teaching us to avoid situations with negative outcomes in the future (Shultz, 2013; Bromberg-Martin et al., 2010).
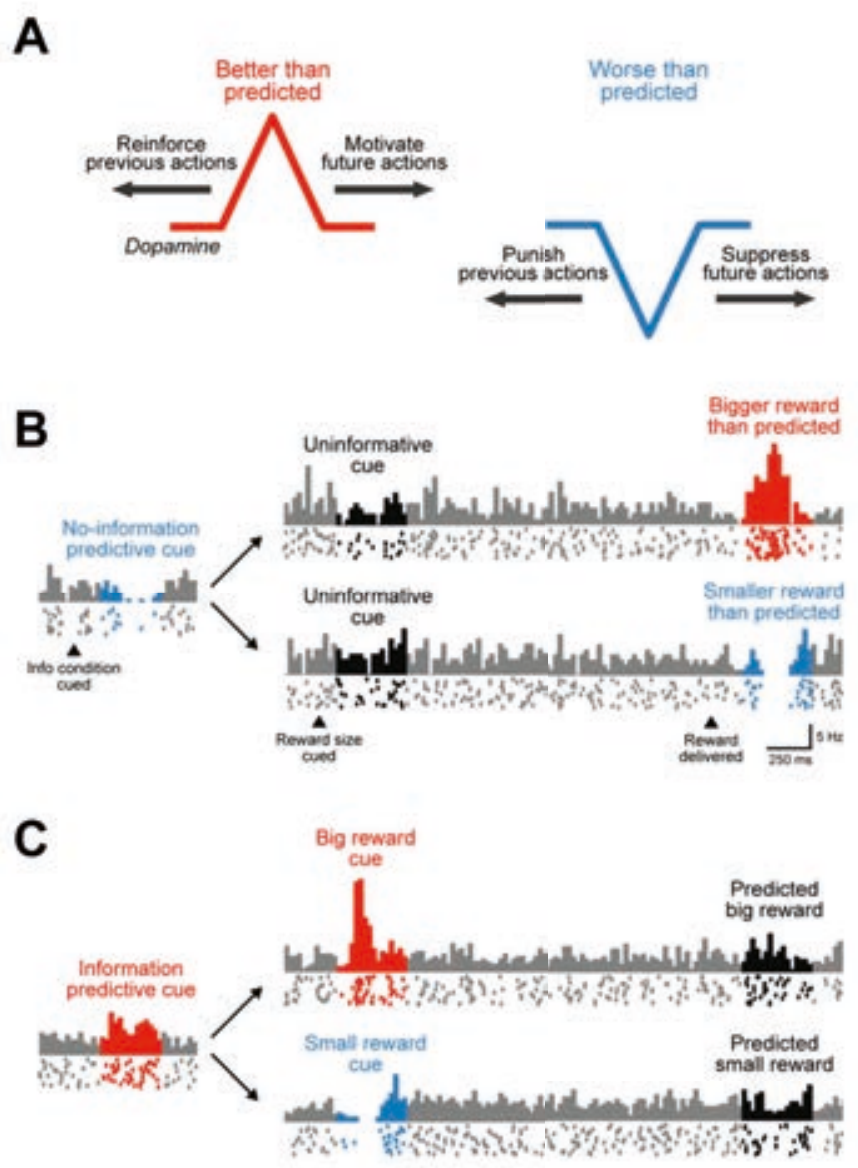

Figure 1. The bi-directional reward prediction error

A. Unexpected positive stimuli or relief make midbrain dopamine neurons burst-fire leading to increased synaptic dopamine, while negative stimuli cause a pause in tonic dopamine firing leading to decreased synaptic dopamine.

B. During the initial phase of conditioning, cues will be uninformative and the unexpected stimuli will immediately elicit a dopamine response according to valence: good = increased dopamine, bad=decreased dopamine. C. Over time and repeated pairing between cues and stimuli, the "dopamine signal" arising from the stimuli will have been transferred to the cue. If the reward or punishment is according to expectation in size and quality, it will no longer elicit a change in dopamine.

Original figure by Bromberg-Martin, Matsumoto and Hikosaka, 2010. Reprinted with permission from Elsevier 


\section{The striatal direct and indirect pathways}

The midbrain dopamine neurons, besides responding to cues in the external environment, evaluate the value of specific stimuli compared to the current internal state in the organism. An example of this could be how the current state of satiety influences the value of a food-reward. The dopamine projections going to the striatum are particularly interesting, as this is the brain region determining how we react to motivational cues in our environment. The striatum is responsible for mapping states and response-options according to specific cues. It functions as the executing unit, as it passes this information on to other brains areas (Takahashi et al., 2008). Ninety percent of the neuronpopulation in the striatum are inhibitory GABAergic medium spiny neurons (MSNs), while only $1-2 \%$ are cholinergic interneurons and the remaining striatal neurons are GABAergic interneurons. The MSNs can be subdivided into two large neural populations: Those that express dopamine D1-receptors (D1Rs) and those that express dopamine D2-receptors (D2Rs). These two types of neurons have opposing responses to dopamine, as the D1Rs are Gs-protein coupled 7-transmembrane receptors (GPCRs), which promote excitatory transmission, while the D2Rs are Gi-coupled and inhibit excitatory transmission. D1R and D2R MSN populations have very different projection-targets and thereby constitute two specific neural pathways named the direct and indirect pathway, respectively. These two pathways can be further subdivided dependent on if the MSNs originate from the ventral $(\mathrm{Nac})$ or dorsal striatum $(\mathrm{CPu})$. In general, direct-pathway D1R MSNs project to midbrain structures (VTA or SNreticulata), whereas indirect-pathway D2R MSNs project to the ventral pallidum (VP)/Globus pallidus external (GPe) and indirectly from there projections reach the midbrain. The main difference between the two pathways is an extra GABAergic connection in the $\mathrm{VP} / \mathrm{GPe}$ of the indirect-pathway. Thereby, the principle of disinhibition ensures that both D1R-MSNs and D2R-MSNs in the presence of dopamine will enforce the same behavioral output (Kravitz and Kreitzer, 2012). Based on the properties of these two pathways, it has been suggested that high levels of dopamine in the striatum, activating D1Rs, will cause the direct pathway to select motivated movements for pursuing rewarding stimuli. Opposing to this, a decrease in dopamine-levels due to a pause in tonic firing will lead to excitability of D2R MSNs and cause the indirect pathway to suppress low-expectancy behaviors and prevent us from approaching aversive stimuli (Bromberg-Martin et al., 2010). This follows the principle of the bidirectional reward prediction error from a volitional movement perspective (Fig. 1, $\mathrm{A}$ and $\mathrm{B})$. 

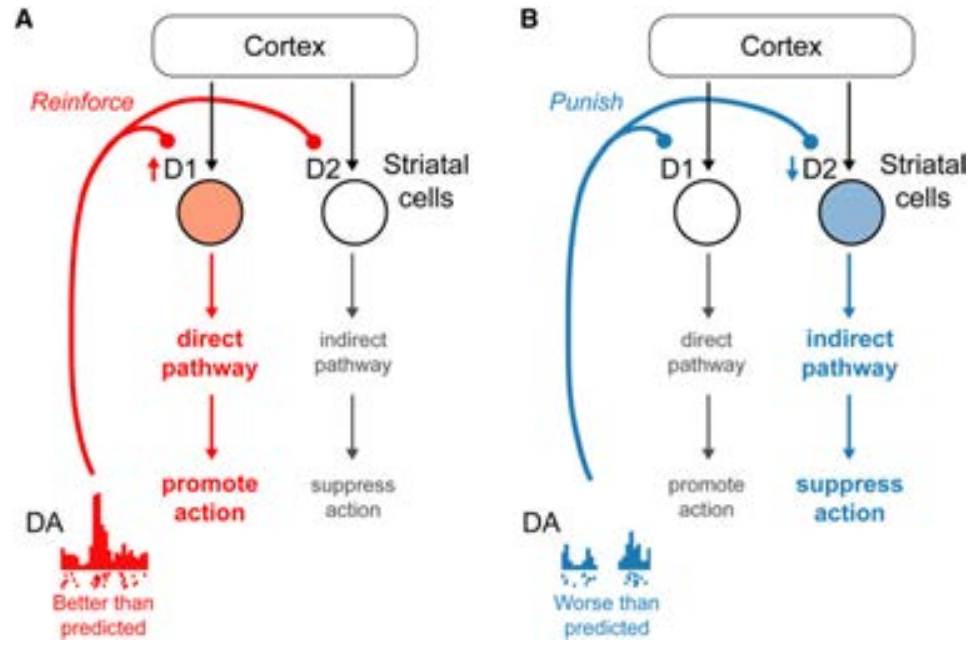

Figure 2. Direct and indirect pathway model describing approach and avoidance behavior in relation to reward-prediction error.

Original figure by Bromberg-Martin, Matsumoto and Hikosaka, 2010. Reprinted with permission from Cell Press

Newer studies employing techniques such as optogenetics (please see technical description under methodological considerations) support the notion that activation of D1R MSNs drive preference behaviors and activation of D2R MSNs decrease reward, motivated movement and mediate aversion (Kravitz et al., 2012).

\section{Systemic inflammation and affective state}

The innate immune system is pivotal for our survival by helping the body combat external pathogens. It immediately responds to a pathogen-challenge by regulating several aspects of our physiology. White blood cells (leukocytes) are specialized in recognizing and initiating responses against invading bacteria and virus. When specialized leukocytes, such as macrophages, identify an intruding pathogen they will respond by producing pro-inflammatory cytokines, including tumor-necrosisfactor-\$ (TNF\$), interleukin-1-\# (IL-1\#), interleukin 6 (IL-6), interferon-" (IFN-"), etc. The cytokines are key mediators of the acute phase of systemic inflammation. This phase is characterized by a variety of physiological symptoms named "the sickness syndrome" and is initiated by immune signaling to the brain. The symptoms include: Fever, loss of appetite, drowsiness/inactivity, hyperalgesia, hypothalamicpituitary-adrenal (HPA) axis activation and social withdrawal. How the peripheral cytokines signal to the brain to elicit these symptoms is a field-controversy, which will be further discussed in the section "signaling across the blood-brain-barrier". Furthermore, systemic inflammation also negatively affects mood and is aversive (Dantzer et al., 2008; Morméde et al., 2003; Fritz and Klawonn et al., 2016; study I). 
Interestingly, scientists have found many similarities between the negative affective and anhedonic state occurring during major depression and the inflammation induced "sickness syndrome", and studies have shown that chronic inflammation leads to negative affect and overt depression via immune-to-brain signaling (Dantzer et al., 2008; Dantzer, 2017). Clinical neuroimaging studies have implied that changes in motivational neurocircuitry and dopamine signaling are major contributors to inflammation induced negative affect, but the results from these reports are conflicting. There exists an equal amount of findings pointing towards inflammation being associated with decreased basal ganglia activity and reductions in dopamine levels, as towards the opposite, as reviewed by Felger (2017). Several factors, ranging from type of inflammatory state to the time point of measurement, could cause this discrepancy. Irrespectively, it is clear that the motivational neurocircuitry is involved in inflammation induced negative affect. At the first glance, the concept of dopamine in negative affective state seems easy to comprehend, but currently it has not been clarified how the dopaminergic neurocircuitry interacts with affective neurocircuitry (such as endogenous opioids and serotonin) during negative mood. As mentioned previously, dopamine is conceptually understood to signal motivational effects, and not the affective traits, of positive and negative stimuli.

Studies investigating the immediate consequence of immune-activation, using healthy human subjects, have demonstrated that acute systemic inflammation leads to decreased activity in basal ganglia and higher depression scores (Reichenberg et al., 2001; Brydon et al., 2008; Eisenberger et al., 2010). The affective consequences of acute systemic inflammation were found to be directly correlational to plasma levels of the pro-inflammatory cytokines TNF $\alpha$, IL-1 $\beta$ and IL-6 (Reichenberg et al., 2001). In this way, the immune-to-mesolimbic system signaling serves an evolutionary purpose by helping us to avoid infections, places and food that make us sick. However, in the case of chronic inflammatory states, this pathway could be detrimental.

\section{Drug addiction}

Drug addiction is a chronic, debilitating disease that affects millions of people around the world. People who are addicted will experience compulsion to seek and take the drug(s) of abuse, loss of control in limiting their drug-intake, and emergence of a negative affective state reflecting withdrawal symptoms (e.g. dysphoria, anxiety, irritability, and pain) in the absence of the drug (DSM-IV; Koob and Le Moal 2006). In this way, addiction is characterized by impulsive and compulsive behavior towards drugs of abuse. No one makes the choice to become an addict or to remain addicted. Drug addicts will typically be caught in a repetitive series of specific behavioral states, which can be described as the three-step cycle of addiction: 
Binge/intoxication is the stage where the individual is consuming the drug of abuse and experiences its rewarding, pleasurable or relieving effects; during withdrawal/negative affect the individual is abstinent (i.e. without the drug) and therefore experiences the negative emotional and physical symptoms related to this; and finally anticipation/cravings describe the stage where the individual is seeking the drug of abuse after abstinence. Essentially, the addiction cycle contains two types of reinforcement: Positive reinforcement linked to drug-induced euphoria and negative reinforcement, linked to the alleviation of negative affective state by the drug of abuse (Koob and Volkow, 2010).

Unfortunately, this disorder is characterized by immense neurobiological complexity, which is not made easier by the differences in mechanisms of various drugs, as well as variations arising from the duration of drug-consumption. Several neurotransmitter systems, hormones and neurotropic factors are involved in the pathology of addiction. Nevertheless, general theories based on empirical observations describing the development of addiction have evolved. Below is given two examples of eminent theories, reward allostasis and incentive sensitization, and an overview of the direct involvement of motivational circuitry in addiction.

\section{Reward allostasis}

The hypothesis of reward-allostasis in drug addiction by George F. Koob and Michel Le Moal is based on Solomon's opponent process theory. The opponent process theory postulates that positive hedonic or motivational states (a-process) will always be modulated by central nervous system (CNS) mechanisms that reduce the intensity of these, leading to a negatively perceived b-process. In this manner, any stimulus that elicits an a-process will also promote an equivalent b-process, which is part of normal homeostatic regulation of reward function. Empirical evidence has exemplified the b-process as a consequence of the activation of brain stress systems, such as those releasing dynorphin, corticotropin releasing factor and noradrenalin (Koob and Le Moal, 2008). But while the a-process is fast and correlates with the characteristics of the stimulus, the b-process is slow in onset, lasts longer and gets larger with repeated exposures (Solomon, 1980).

Koob and Le Moal expanded the opponent process theory, by suggesting that an "allostatic model" of the motivational system explains the persistent changes in motivation associated with drug dependence. In this context allostasis can be defined as the ability to achieve stability through change. The idea is, that in the case of drugs-of-abuse the a-process will be unnaturally large and in return cause an equally large b-process. Over time, when retaking the drug, this will lead the opponent bprocess to fail to return to its normal set-point and in this manner make the individual more prone to consume the drug of abuse for relief. This allostatic state represents a 
deviation from the reward set point, caused by the brain and hormonal stress responses. In this way opponent motivational processes of drug consumption are responsible for the negative reinforcement driving the addict in a downward spiral (constituted by the three step circle of behaviors) into further compulsive consumption (Koob and Le Moal, 2008).
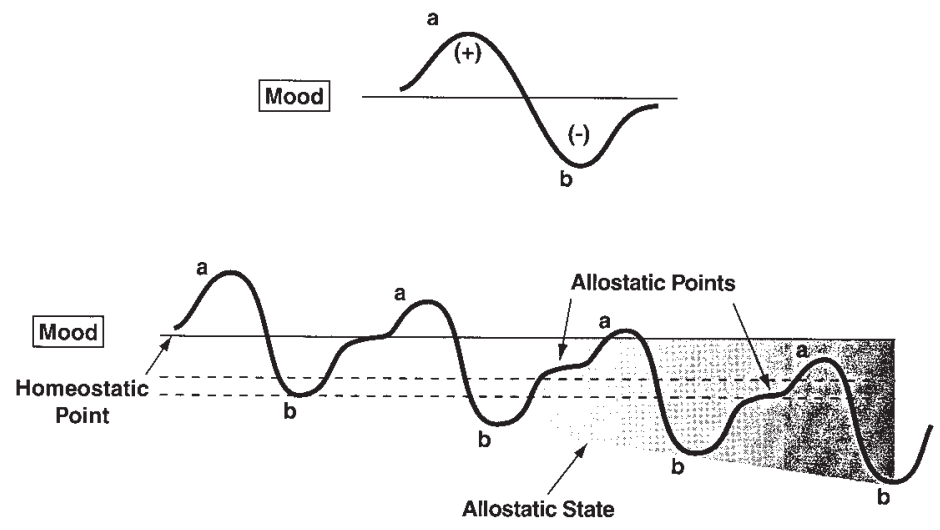

Figure 3. The opponent process: By nature every motivational or affective a-process has an equivalent b-process. During addiction an allostatic state occurs which represents a deviation from the reward set point. This is caused by changes in the brain and hormonal stress response.

Original figure by Koob and Le Moal, 2000. Reprinted with permission from Elsevier

\section{Incentive sensitization}

Terry E. Robinson and Kent C. Berridge postulated that the compulsive drug consumption that characterizes addiction is not motivated by the pleasure associated with the intake. In contrast to Koob and LeMoal, they argue that seeking drugs of abuse is unrelated to the desire to relieve withdrawal symptoms (the b-process). Instead it is the consequence of "incentive sensitization". Incentive sensitization occurs due to the long lasting changes in motivational neurocircuitry induced by drug consumption. These neuroadaptations render the brain reward system sensitized to drugs and drug-associated stimuli (cues). Consistent with the role of dopamine, these changes only affect incentive salience, the motivational aspect of drug wanting, but not the hedonic sides, i.e. the drug liking. Hence, incentive sensitization depicts the process of sensitization of incentive salience signaling specifically associated with the drug of abuse (Robinson and Berridge, 2001). Since this theory was proposed in 1993, several studies have emerged supporting the idea of changes in 
synaptic connectivity of the motivational neurocircuitry as the cause of drug addiction (as reviewed by Lüscher and Malenka, 2011), and in 2001 it was reported for the first time that a single cocaine exposure changes the plasticity of dopamine neurons in vivo (Ungless et al., 2001).

\section{Dopaminergic circuitry and striatal pathways}

All drugs of abuse share on thing in common: They increase mesolimbic dopamine levels. To use the example most relevant for the present work, the psychostimulant cocaine is a potent dopamine transporter (DAT) blocker and elevates dopamine in the synapse by preventing reuptake after neurotransmitter-release. In this way, it has been suggested that drugs of abuse produce pathologically stronger reward prediction errors (Keiflin and Janak, 2015). Preclinical studies have shown that the critical difference between natural and drug rewards, resides in the dopamineresponse they elicit. Whereas food causes a time-locked dopamine signal, which decreases as reward becomes expected, cocaine prompts a delayed long-lasting increase in the frequency and amplitude of dopamine transients (Heien et al., 2005; Stuber et al., 2005; Keiflin and Janak, 2015). Hence, with repeated drug-use, the pharmacological dopamine signal will continue to reinforce the drug-related cues. According to this hypothesis, drugs of abuse will bias future decision-making towards drug choice. These initial changes in the dopamine levels will inevitably affect direct and indirect pathway neurons of the striatum. Yet, the question of the involvement of the direct and indirect pathway in the development of addictive disorders is ambiguous. Imaging studies on abstinent human subjects addicted to various kinds of substances of abuse showed decreases in D2R binding (Volkow et al., 2007, Martinez et al., 2004; 2005). This may be the consequence of long-term drug-exposure rather than the initiating factor of addiction. On the other hand, studies show that subordinate monkeys and people from poor socioeconomic backgrounds have lower levels of D2R binding (Grant et al., 1998; Morgan et al., 2002; Martinez et al., 2010). More importantly, there exist a strong link between low socioeconomic status and likelihood of substance abuse (Gauffin et al., 2013), and in line with this, socially inferior animals consume more drugs of abuse (Morgan et al., 2002). These findings indicate that negative affective state, arising from social hopelessness and despair, influences the indirect pathway signaling, which in turn could affect the likelihood of developing addiction. At the same time, several preclinical studies point towards D1-receptors (the direct pathway) being important for the initial cue-associated learning in addiction. In the present work, the role of muscarinic M4 receptors on direct-pathway MSNs in impulsive and cocainereinforcement behaviors has been investigated (study IV). 


\section{IMMUNE-TO-BRAIN SIGNALING}

\section{Cytokines in motivation and affective state}

The release of pro-inflammatory cytokines influences a broad spectrum of cell types in the immune system. As explained previously, specialized leukocytes of the innate immune system, such as activated monocytes and macrophages, are responsible for the fast production of peripheral pro-inflammatory cytokines upon immunestimulation (Kindt, Goldsby and Osborne, 2007; Dantzer, 2001). The brain was long considered an "immune-privileged" organ, which implied that it was isolated from the immune system and unable to elicit an inflammatory response. This was primarily based on the function of the blood-brain barrier (BBB) in shielding the brain from non-soluble immune-factors, such as pro-inflammatory cytokines.

We now know this view was an oversimplification, as the peripheral immune system has several pathways available for signaling to the brain - some of these will be clarified in the following sections. Furthermore, the brain has its own type of parenchymal (tissue) macrophages, the microglia. These cells respond to peripheral immune signaling with production of pro-inflammatory cytokines, and create in this way a brain mirror image of the cytokine-profile of the peripheral immune system. The main difference of this image, compared to that of the periphery, is that it does not involve an invasion of immune cells into the parenchyma and is not distorted by tissue damage at the site of infection (Dantzer et al., 2008).

\section{IL-1及 signaling}

The interleukin-1 (IL-1) family comprises 11 different members, which despite structural homology exert substantially different biological functions (Schett, Dayer and Manger, 2016). The most studied of the IL-1 cytokines in pro-inflammatory signaling are IL-1 $\beta$ and IL-1 $\alpha$, and the IL-1-receptor specific antagonist (IL-1Ra). Though IL- $1 \alpha$ binds to the same receptors as IL- $1 \beta$, it is not actively secreted from innate immune cells during systemic inflammation; instead it is part of the inflammatory response to necrosis (Chen et al., 2007). IL-1 $\beta$ production is initiated by transcription of an IL-1-precursor protein gene, which final protein-product is cleaved by specific intracellular enzymes for the formation of IL-1 $\beta$. In monocytes and macrophages, the cleavage is dependent on inflammasome (a multiprotein oligomer) formation, and the enzyme caspase 1, which is part of the inflammasome (Martinon et al., 2002; Wilson et al., 1994; Thornberry et al., 1992).

IL-1 $\beta$ signaling is complex and involves strict negative control mediated by both IL$1 \mathrm{Ra}$ and one of its receptors. IL-1 $\beta$ is able to bind to two receptors: IL-1- receptor (IL-1R) type 1 and type 2 . The IL-1R type 1 is mediating signal transduction, while IL-1R type 2 has suppressive functions, as its short cytoplasmic tail is unable to 
induce signaling (Re et al., 1996). Hence the IL-1R type 2 functions as a decoy that binds excess IL-1 $\beta$. The natural IL-1R antagonist IL-1 Ra is expressed in virtually all tissue and thereby competitively prevents uncontrolled activation of the IL-1R (Dinarello, 2000).

When IL-1 $\beta$ binds to IL-1R type 1 , it will then interact with the IL-1 receptor accessory protein (IL-1RAP) to induce signal transduction. The functional IL-1R/IL1RAP heterodimer will recruit the adaptor protein myeloid differentiation factor 88 (MyD88), which can further recruit the signaling molecules IL-1 receptor-associated kinase 1 (IRAK1) and 4 (IRAK 4), and TNF $\alpha$ associated factor 6. This will result in the activation of several downstream transcription factors and protein-kinases that will initiate the executive actions of IL- $1 \beta$ signaling (e.g. nuclear factor $\kappa B(N F \kappa B)$, p38, c-Jun N-terminal kinase (JNK), extracellular signal-regualted kinase (ERK) and mitogen-activated protein kinase (MAPK)) (Schett, Dayer and Manger, 2016). Interestingly, compensatory interactions between $\mathrm{TNF} \alpha$ and IL-1 $\beta$ signaling have been demonstrated. During functional loss of IL-1 $\beta$-signaling via IL-1R type 1 knockout, TNF $\alpha$ has been reported to substitute for its absence in mediating various sickness symptoms in response to systemic inflammation (Bluthé et al., 2000).

\section{Myd88 and Toll-like receptors}

Myd88-dependent signal transduction is also utilized by other pro-inflammatory signaling receptors, such as the Toll-like receptors (TLRs). Toll-like receptors are pattern-recognition receptors (PRRs) responsible for recognizing pathogenassociated molecular patterns (PAMPs) on microorganisms and initiating the innate immune response. 11 TLRs have been discovered in the human genome, and 13 in the mouse, responsible for recognition of everything from mycobacteria to virus (O'Neil, Golenbock and Bowie, 2013). TLR4 is particular relevant, as it binds to the membrane component lipopolysaccharide (LPS) from gram-negative bacteria, such as Escherichia Coli (E. coli), which is used as the primary model to induce systemic inflammation in the present work (study I, II and III) (Schett, Dayer and Manger, 2016). The importance of Myd88-signal transduction for initiating an immuneresponse is clear, since human beings deficient of Myd88 are immune-compromised and more susceptible to disease, while Myd88 knockout mice are unable to mount immune-responses to a number of pathogens (19 bacteria, 7 viruses, 5 parasites and 4 fungi) (von Bernuth et al., 2008).

\section{IL-1B in motivation and affective state}

Though it is difficult to determine affective and motivational effects of IL- $1 \beta$ in preclinical models, due to sickness induced changes in metabolism and locomotion, different self-administration protocols have been designed to adress these issues. For 
instance, low doses of systemic IL-1 $\beta$ have been demonstrated to decrease motivation to obtain food-reward, without reducing baseline food-intake (Nunes et al., 2014), while it has been shown that treatment with the antidepressant fluoxetine is capable of blocking IL- $1 \beta$ mediated reduction in motivation to obtain food-reward in a progressive ratio paradigm, without altering food-intake (Merali et al., 2003). Furthermore, systemic IL-1 $\beta$ has been demonstrated to induce both taste and place aversion in rodents (Tazi et al., 1988; Morméde et al., 2003). IL-1 $\beta$ signaling has also been implied in anxiety behaviors, as repeated administrations of low doses of IL-1 $\beta$ induce anxiety (Sokolova et al., 2007), while overexpression of the natural IL$1 \mathrm{R}$ antagonist, IL-1Ra, has anxiolytic effects (Oprica et al., 2005). Several animal models of depression have demonstrated elevated IL- $1 \beta$ levels in the blood (Hodes et al., 2015). Finally, increased levels of IL-1 $\beta$ have been linked to negative affective state associated with inflammatory diseases in human beings (Rossi et al., 2017; Bouchard et al., 2016; Liebregts et al., 2007) and serum levels of IL-1 $\beta$ are elevated in patients with major depression (Hannestad et al., 2011). Interestingly, selective serotonin reuptake inhibitors (SSRIs) exhibit anti-inflammatory effects by reducing circulating IL-1 $\beta$ (Hannestad et al., 2011).

\section{TNF $\alpha$-signaling in motivation and affective state}

The TNF $\alpha$ superfamily comprises 19 structurally related cytokines that in the same manner as the IL-1 family exert very different functions. TNF $\alpha$ is the prototypic member of the TNF $\alpha$ superfamily and is in the same manner as IL-1 $\beta$ a key player in initiating the acute phase response of inflammation (Dantzer et al., 2008). TNF $\alpha$ is primarily produced as transmembrane proteins arranged in stable homotrimers. The membrane-bound TNF $\alpha$ can also be cleaved from cells to form soluble sTNF $\alpha$, which function is still considered controversial (Wajant et al., 2003; Croft and Siegel, 2017). Macrophages are the primary source of TNF $\alpha$, but other cell-types such as lymphocytes, mast cells, endothelial cells and CNS microglia also produce TNF $\alpha$ (Wajant et al., 2003; Yu et al., 2017). TNF $\alpha$ binds to two membrane-bound receptors: The TNF $\alpha$ receptor type 1 and 2 (TNF $\alpha \mathrm{R} 1 / 2)$. TNF $\alpha \mathrm{R} 1$ is expressed in most cell-types throughout the body, whereas $\mathrm{TNF} \alpha \mathrm{R} 2$ is more specifically expressed in immune cells of the lymphoid system. TNFaR1 and 2 have long cysteine-rich repeats, which interact with the lateral groove of the membrane TNF $\alpha$ trimers (Banner et al., 1993). Furthermore, the extracellular domains of both $\mathrm{TNF} \alpha \mathrm{R} 1$ and 2 can be cleaved forming soluble receptors, which retain the ability to bind TNF $\alpha$ and thereby compete with membrane-receptor binding (Van Zee et al., 1992). On a more general note TNF $\alpha \mathrm{R} 1$ mediated signal-transduction involves

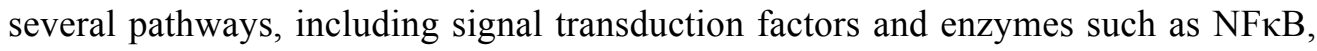


JNK, ERK, protein kinase C (PKC) and MAPK. A description of these signaltransduction pathways are beyond the scope of this thesis; the interested reader is referred to reading reviews from Wajant, Pfizenmaier and Scheurich (2003) or Croft and Siegel (2017).

Hardly any studies have investigated effects of TNF $\alpha$ on motivated behaviors, but it has been demonstrated that systemic TNF $\alpha$ injections, in the same manner as IL-1 $\beta$, induce conditioned taste aversion in rats (Goehler et al., 1995). Several preclinical studies have found TNF $\alpha$ to be associated with elevated anxiety behavior both during baseline conditions and systemic inflammation (Simen et al., 2006; Silverman et al., 2007). Interestingly, in humans TNF $\alpha$ is one of the most relevant cytokines implied in psychopathologies. Meta-analyses of a broad array of studies have demonstrated that peripheral $\mathrm{TNF} \alpha$ is significantly upregulated in patients with major depressive disorder (Haapakoski et al., 2015; Dowlati et al., 2010). Furthermore, TNF $\alpha$ is, in the same manner as IL-1 $\beta$, associated with depressive symptoms during inflammatory disease. Treatments targeting $\mathrm{TNF} \alpha$-signaling in inflammatory conditions have proven to be useful against the associated negative affective symptoms (Kappelman et al., 2016). In the preclinical literature, both peripheral and central TNF $\alpha$ has been demonstrated to play a role in the development of depressive symptoms (Kaster et al., 2012; O'Conner et al., 2009b).

Preclinical studies have suggested that depressive symptoms, arising from inflammation induced $\mathrm{TNF} \alpha$, are due to the enzyme Indoleamine-2,3-deoxygenase (IDO) in the CNS. Both IFN- $\gamma$ and TNF $\alpha$ lead to gene-induction of IDO, and upregulation of its activity. Blocking IDO induction, by decreasing TNF $\alpha$ or IFN- $\gamma$ signaling, is efficient for preventing the development of inflammation-induced depression (O'Conner et al., 2009b). Furthermore, functional studies have demonstrated that deletion of IDO counteracts inflammation induced depression-like behavior in the forced swim test paradigm (O'Conner et al., 2009c). IDO is the ratelimiting enzyme in the catabolism of tryptophan as part of the kynurenine pathway. Although the specific mechanism by which IDO signals negative affect is not yet clarified, it has been suggested that metabolites of the kynurenine pathway, such as kynurenic and quinolinic acid, may be key-players. Microglial cells primarily produce quinolinic acid, whereas kynurenic acid is synthesized by astrocytes. Both quinolinic and kynurenic acids influence glutamatergic neurotransmission via $\mathrm{N}$ methyl-D-aspartate (NMDA) receptors (Dantzer, 2017). 


\section{IFN $\gamma$-signaling in motivation and affective state}

The cytokine IFN- $\gamma$ belongs to the family of type II interferons. The type I interferons include 13 subtypes, while IFN- $\gamma$ is the only type II interferon. These cytokines are the first line of defense against viral infections, but are also generally released during the acute phase response of systemic inflammation (Dantzer et al., 2008; Platanias, 2005). IFN- $\gamma$ does not share marked structural homology with the type I interferons and it binds to a different receptor - the type II Interferon receptor, IFN- $\gamma$ R. It was included in the interferon-family due to its anti-viral properties. Irrespectively, there exist some common features of IFN-signal transduction; both types of interferons utilize the JAK-STAT pathway. The IFN- $\gamma \mathrm{R}$ has two distinct subunits (IFNGR1 and IFNGR2), which each interact with a tyrosine kinase, Janus activated kinase (JAK). IFNGR1 associates with JAK1 and IFNGR2 is constitutively associated with JAK2. Upon binding of IFN- $\gamma$ the two subunits will dimerize, which brings the JAKs closer together and causes cross-phosphorylation, and subsequent activation. When active, the JAKs typically phosphorylate the transcription factor STAT, which leads STAT to form an active dimer that can translocate to the cell nucleus (Platanias, 2005). In this manner IFN- $\gamma$-signaling can regulate the expression of a broad array of genes, including the ones expressing pro-inflammatory signaling molecules such as Cxcl10/IP10 (Interferon- $\gamma$ Protein 10) (Majumder et al., 1998). The JAK-STAT is a central IFN- $\gamma-\mathrm{R}$ signaling route, but the receptors have also been found to recruit various other pathways, both dependently and independently of STAT (Platanias, 2005). Interestingly, IFN- $\gamma$-Rs were demonstrated to recruit Myd88, leading to stabilization of gene-transcripts arising from IFN- $\gamma$-R-signaling (Sun and Ding, 2006).

IFN- $\alpha$ has received much more attention than IFN- $\gamma$ in negative affective state. The focus on pro-inflammatory cytokines in psychopathologies was boosted by the high occurrence of major depressive disorder as a consequence of IFN- $\alpha$ treatment against hepatitis C (Lotrich, 2009). Recently, IFN- $\alpha$ mediated depressive behavior in mice was demonstrated to involve a mechanism similar to the ones described in study I and II. IFN- $\alpha$ was shown to induce negative affective state via receptors specifically on BBB endothelial cells leading to increased release of the chemokine Cxcl10, which in turn binds to Cxcr3-receptors on neurons (Blank et al., 2016). In this context, it is worthwhile noting that the Cxcr3-receptors are expressed on both neurons and microglia (Blank et al., 2016). This model offers a very direct mechanism through which interferons can cause negative affect. However, another molecular pathway has been suggested for IFN- $\gamma$ induced depressive behaviors. Preclinical studies have suggested that inflammatory IFN- $\gamma$ signaling results in activation of microglial IDO (in the same manner as TNF $\alpha$ does, as described above) (Myint et al., 2013; Mahmoud et al., 2017; O’Conner et al., 2009). 


\section{Signaling across the blood-brain-barrier}

It is clear that the peripheral pro-inflammatory cytokines play an important role in mediating changes in motivational and affective state. As mentioned previously, how the peripheral cytokines signal to the brain to elicit these symptoms is a field of controversy. The brain is protected from the blood by the BBB, which consists of endothelial cells connected by tight junctions and a layer of their extracellular matrix (basal lamina). The pro-inflammatory cytokines are large hydrophilic peptides that are unable to traverse the $\mathrm{BBB}$, hence there must exist other mechanisms through which their signal can reach the brain.

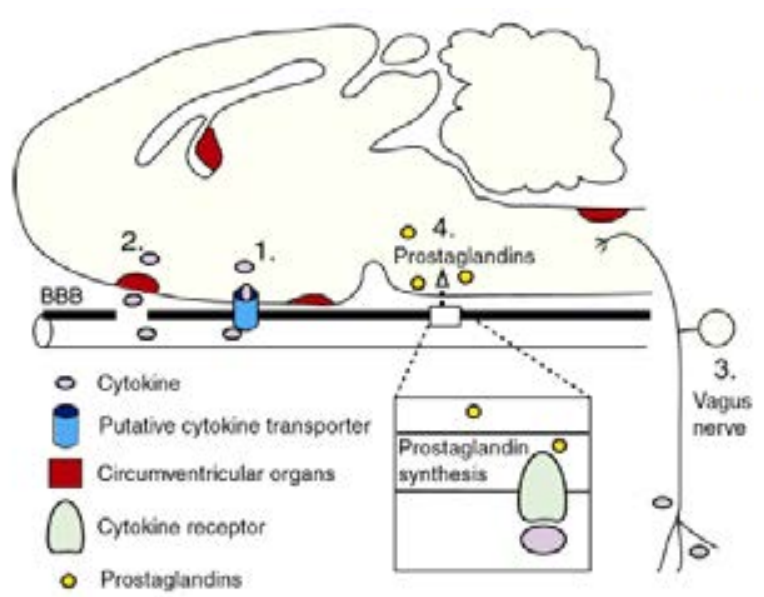

Figure 4. Immune-to-brain signaling across the blood brain barrier $\mathbf{- 4}$ potential pathways 1. Cytokines can cross the BBB via active transport. 2. Circumventricular organs are areas without functional BBB, where cytokines can enter the brain. 3. Cytokine receptors activate vagus nerve signaling to brainstem nuclei. 4. Cytokine receptors on the BBB endothelial cells induce Prostaglandin E2 (PGE2) synthesis. PGE2 is released directly from the endothelium into the brain where it can activate neural EP-receptors.

Several mechanisms for signal-transfer across the BBB have been suggested; for an overview of these see figure 4 . The four most central hypotheses are:

1) Transporters on the BBB could provide direct entry of circulating cytokines into the brain. Such transport has been described for IL-1\#, TNF\$ and IL-6 (Banks, 2015). The proteins responsible for the cytokine transport have not yet been characterized. Furthermore, as the systems are saturable they are slow and therefore less likely to induce the rapid effects for cytokines during systemic inflammation (Banks et al., 1995). 
2) Areas devoid of a BBB provide direct access for pro-inflammatory cytokines to the brain. The circumventricular organs (CVOs) are structures that surround the brain ventricles and that lack functional BBB due to fenestrated capillaries. Furthermore, macrophages and microglia are known to reside in the CVOs (Dantzer et al., 2008). There are four CVOs (area postrema, suprafornical organ, median eminence and organum vasculosum of the laminae terminalis), which are located around the third and fourth ventricles of the brain. It remains unknown whether cytokines from the third ventricle can reach brain areas near the lateral ventricle. Interestingly, the choroid plexus, which is responsible for the production of cerebrospinal fluid, responds to peripheral PAMPs by expressing pro-inflammatory cytokines (Quan et al., 1998).

3) Cytokine-receptors have also been found on peripheral nerves, such as the vagus nerve. The vagus nerve is activated by pro-inflammatory cytokines and projects directly to brain-stem nuclei, such as the NTS (Ek et al., 1998). Vagal activity has in particular been implied during abdominal and visceral infections (Dantzer et al., 2008). Unfortunately, the functional role of the vagus nerve in systemic inflammation induced sickness and negative affect remains to be clarified. Results from vagotomized rats and mice are difficult to interpret, as this type of experimental procedure leaves the animals with severe physical complications, such as gastrointestinal dysfunction (Saper, Romanovsky and Scammel, 2012).

4) Cytokine-receptors localized directly on BBB endothelial cells have been demonstrated to transduce a second-messenger signal to neurons. These receptors stimulate synthesis of prostaglandin E2 (PGE2), which is preferentially released on the basal side of the endothelial cell and target prostaglandin receptors on neurons (Ek et al., 2001; Engblom et al., 2003; Ching et al., 2007; Wohleb et al., 2014). It is likely that cytokine or PAMP binding to receptors on the BBB endothelial cells can facilitate release of other types of messenger molecules. For instance, it has been shown that endothelial cells from other parts of the body release various proinflammatory molecules, including monocyte chemoattractant protein-1 (MCP-1) (Schratzberger et al., 1998). This type of signaling could represent a whole new path for recruitment of microglia within the brain. 


\section{Prostaglandin E2 synthesis and receptors}

Prostaglandin E2 is a key player in inflammation signaling. Inhibition of PGE2 synthesis has been a major anti-inflammatory strategy throughout the past 100 years by use of common NSAIDs (non-steroidal anti-inflammatory drugs) such as aspirin and salicylate.

PGE2 is synthesized from membrane phospholipids. The enzyme Phospholipase A2 cleaves membrane phospholipids by hydrolysis, leading to the release of arachidonic acid. Subsequently, cyclooxygenase enzymes convert the arachidonic acid through two steps (oxygenation and reduction) to the unstable prostanoid Prostaglandin H2 (PGH2). There exist two genetically distinct isoforms of the cyclooxygenases: COX1 and COX2. COX1 is in general a constitutive enzyme, but has been demonstrated to be upregulated during certain inflammatory conditions (Schwab et al., 2000; Shukuri et al., 2011; Anrather et al., 2011; Matousek et al., 2010). The COX2 gene on the other hand has several transcriptional regulatory sites in its promoter and can be induced by various pro-inflammatory cytokines. Both enzymes reside at the endoplasmatic reticulum (Park et al., 2006). There exist other splice variants of COX1, but their functions in inflammation remains to be clarified. Brain COX1 expression is strongest in microglia, but it is also expressed in endothelial cells (Tanaka et al., 2012; Garcia-Bueno et al., 2009). COX-2 is expressed in neurons of the cortex and hippocampus, but is otherwise not strongly expressed under basal conditions. In response to systemic inflammation a strong induction takes place primarily in endothelial cells (Cao et al., 1995). The unstable product of the cyclooxygenases, PGH2, serves as substrate for various specific enzymes that produce more stable prostanoid species, among others PGE2. There exist three specific PGE2 synthases: microsomal PGES-1 and- 2, and cytosolic PGES. mPGES1 is the main isomerase responsible for inflammation induced PGE2 (Jakobsson et al., 1999). Studies have suggested that mPGES1 primarily couple to COX-2 over COX1, but other findings demonstrate exceptions to this rule, and mPGES1 has been found to couple to COX1 in cases where the concentration of arachidonic acid is high (Matousek et al., 2010; Chandrasekharan et al., 2005; Murakami et al., 2000).

COX2 provides the main source of prostaglandin E2 production mediating inflammatory symptoms such as fever and loss of appetite (Wilhelms et al., 2014; Nilsson et al., 2017a) and in particular endothelial mPGES1 is important for the pyrogenic response to inflammation (Ek et al., 2001; Engblom et al., 2003; Wilhelms et al., 2014). In comparison, COX 1 is responsible for PGE2 leading to social defeat stress, which has been implied to involve microglial activation (Tanaka et al., 2012), and COX-1 expression in endothelial cells has been suggested to drive the early phase of corticosterone release during systemic inflammation (Elander et al., 2009; Garcia-Bueno et al., 2009). Interestingly, mPGES-1 is co-induced with COX-1 
mediating PGE2 elevation in the hippocampus during long-term IL-1 $\beta$-mediated inflammation (Matousek et al., 2010).

Four different GPCRs have been discovered which are activated by PGE2: EP1-EP4 receptors. These have been found to have primary functions in signaling specific aspects of sickness during systemic inflammation. EP1Rs are Gq-coupled receptors and widely expressed throughout the brain of rodents, but in particularly in forebrain structures, including striatum and prefrontal cortex, and in the brain stem (Candelario-Jalil et al., 2005). The EP1Rs are expressed on both direct (D1R) and indirect (D2R) pathway neurons in the striatum (Kitaoka et al., 2007), as well as on dopaminergic neurons in SN (Tanaka et al., 2009). In line with this, EP1Rs have been shown to increase GABA-mediated inhibition of dopaminergic neurons in the SN (Tanaka et al., 2009). The EP1R mediated effect on dopaminergic transmission is important for mediating affective and motivational behaviors such as social defeat stress and impulsivity in mice (Tanaka et al., 2012; Matsuoka et al., 2005). EP2Rs are Gs-coupled receptors and are also expressed throughout the brain. Interestingly, in particular forebrain structures express EP2Rs and this expression is upregulated by systemic inflammation in structures related to affective state, such as the lateral septum, BNST, cortex and amygdala (Zhang and Rivest, 1999). The EP2Rs have not until recently received any major attention, but there exist a few studies that have implied these receptors in hyperalgesia (Ota et al., 2017). EP3Rs are primarily Gicoupled receptors that are responsible for the pyrogenic response to systemic inflammation (Ushikubi et al., 1998; Lazarus et al., 2007). They have been found throughout the brain of rodents, with particularly high expression levels in the median preoptic hypothalamus and in thalamic nuclei, but also in areas such as the hippocampus, septum and amygdala, as well as in brainstem nuclei such as NTS and the parabrachial nucleus (Ek et al., 2000). In particular the EP3Rs of the preoptic hypothalamus have been found to be responsible for signaling inflammatory fever (Lazarus et al., 2007). The EP3Rs also play a role in signaling PGE2 mediated hyperalgesia and in the affective component of pain (Minami et al., 2001; Singh et al., 2017). EP4Rs are Gs-coupled which are widely expressed throughout the brain of rodents. In particular the PVH, cerebellar cortex and brainstem structures exhibit high levels of EP4Rs in rodents (Zhang and Rivest, 1999), but the role of EP4Rs in immune-to-brain signaling during systemic inflammation is largely unknown. 


\section{MELANOCORTINS IN MOTIVATION AND AFFECTIVE STATE}

Food intake and motivational valence are by nature intricately linked. The evaluation of a food reward and motivation to pursue it rely on the same neurocircuitry (i.e. the mesolimbic system) as all other types of salient stimuli. The loss of control over food consumption in obese people who struggle to maintain a healthy weight is in many ways like the compulsive character traits associated with drug abuse. On the other hand, during inflammation induced sickness and other aversive states, such as pain and stress, there naturally occur a shift in the motivational valence associated with food. These examples demonstrate how the circuitry regulating satiety essentially is linked with our perception of good and bad.

\section{The arcuate nucleus melanocortin circuitry}

The arcuate nucleus of the hypothalamus (ARC) is known for its role in monitoring homeostatic states related to caloric energy balance, and regulating appropriate behavioral responses to changes in these. Two neuropopulations with dichotomous functions are responsible for maintaining the delicate equilibrium between energyintake and satiety. Not too surprisingly, these are direct targets of the major hormonal pathways responsible for regulating metabolism and food-intake, such as the peripheral leptin, insulin and ghrelin signaling pathways (Krashes, Lowell and Garlfield, 2016). The ARC neurons expressing the agouti-related peptide (AgRP) and co-expressing neuropeptide $\mathrm{Y}$ and GABA are responsible for anabolic processes, i.e. promoting increased food-consumption for building up energy-storage. This signaling is under normal circumstances stimulated by caloric insufficiency, in order to drive food intake, while conserving energy expenditure and promoting weightgain. In contrast, the proopiomelanocortin (POMC) expressing neurons of ARC are responsible for promoting satiety and cessation of feeding, in combination with increasing energy expenditure and weight loss. The functional transmitters of POMC-neurons are the bioactive products $\alpha-\beta$ - and $\gamma$ - melanocyte stimulation hormones; these are part of the melanocortin-peptides, which arise from POMCprocessing (Krashes, Lowell and Garfield, 2016). Subpopulations of ARC POMC neurons have also been reported to either co-release the neurotransmitter GABA or Glutamate (Atasoy et al., 2014). The ARC POMC and AgRP neurons target most of the same brain areas, with the strongest innervation occurring in hypothalamic structures (such as the paraventricular hypothalamic nucleus $(\mathrm{PVH})$, lateral hypothalamus, medial preoptic nucleus, dorsomedial hypothalamus) and the paraventricular thalamic nucleus (Wang et al., 2015; Betley et al., 2013; Atasoy 2008). Multiple studies have revealed that the appetite reducing effect of melanocortin signaling primarily is mediated by ARC POMC-neurons projecting to 
the PVH (as reviewed by Krashes, Lowell and Garfield, 2016). Interestingly, the use of modern techniques such as chemogenetics and optogenetics for activation of ARC AgRP and POMC neurons have demonstrated different temporal dynamics of this circuitry. For instance, AgRP-activation leads to immediate consummatory behavior, whereas POMC-neurons produce late onset responses (Aponte et al., 2011; Atasoy et al., 2012; Zhan et al., 2013). Though ARC is considered the primary site for melanocortin synthesis, other structures contain POMC neurons as well, such as the nucleus of the solitary tract (NTS). The NTS is primarily involved in encoding gustatory and visceral information. Activation of the NTS POMC population produces anorexigenic effects (Zhan et al., 2013), in the same manner as ARC POMC neurons.

Recent studies indicate that the molecules and circuits controlling energy balance also regulate the affective state (Liu, Lee and Elmqvist, 2014). Both POMC and AGRP neurons target neurocicruictry involved in motivation and affective state, such as the bed nucleus stria terminalis (BNST), central amygdala and NAc (Wang et al., 2015; Betley et al., 2013; Atasoy et al., 2008). Recently it was discovered that optogenetic activation of ARC AgRP-neurons leads to aversion, and that these neurons directly respond to cues associated with food by decreasing their activity (Betley et al., 2015). Other studies have implied a role for POMC-derived melanocortins in signaling negative affect, such as anxiety and depression (Lim et al., 2012; Liu et al., 2013; Goyal et al., 2006; Kokare et al., 2005). Intracerebroventricular (i.c.v) or intra-amygdala injections of melanocortins in the CNS triggers stress signaling, including HPA-axis activation and induction of corticotropin releasing factor expression (Lu et al., 2003; Liu et al., 2013).

Collectively, these findings have demonstrated that circuitry encoding satiety and homeostasis also play a role in motivational valence and affect, though the work on elucidating their exact function is still in its early phase.

\section{Melanocortin 4 receptors}

The mammalian genome encodes 5 melanocortin receptors, but only the melanocortin 3 and 4 receptors are expressed in the CNS. The role of the MC3R in satiety and motivation remains to be further clarified. The MC4R is known to be a cornerstone in appetite and energy balance control. MC4Rs are activated by $\alpha$ melanocyte stimulating hormone $(\alpha-\mathrm{MSH})$ released from POMC neurons, while AgRP acts as an antagonist (Krashes, Lowell and Garfield, 2016). MC4R mutations leading to deficient signaling is the most prevalent monogenic cause of obesity in human beings (Farooqi et al., 2003). MC4Rs are GPCRs, which promote excitation, but various studies have identified different ways through which this can occur. MC4R agonists have been demonstrated to promote Gs-dependent cAMP synthesis, 
Gq-dependent phospholipase activity and small GTPase associated MAPK-signaling (Gantz et al., 1993; Daniels et al., 2003; Vongs et al., 2004). Interestingly, a recent study uncovered a G-protein independent mechanism in PVH-neurons, where MC4Rs recruit the inward rectifying potassium channel Kir7, which leads to Kir7closure and thereby depolarization (Ghamari-Langroudi et al., 2015). Based on these findings it has been suggested that the MC4Rs may be promiscuous between different G-protein and nonG-protein pathways (Krashes, Lowell and Garfield, 2016). The MC4Rs are present throughout the brain in areas targeted by AgRP and POMC neurons (Liu et al., 2003; Kishi et al., 2003), and in this manner are expressed on both circuitry involved in satiety regulation and energy homeostasis, but also on neurons regulating motivation and affective state.

It has previously been suggested that melanocortin signaling plays a role in sicknessinduced aversion, and MC4Rs have been demonstrated to be involved in inflammation induced appetite loss (Marks et al., 2001). In this context, it is relevant that i.c.v. injections of melanocortins elicit conditioned taste aversion (Thiele 1998; Benoit et al., 2003). Furthermore, a few studies have implied central MC4Rs as important for pain perception. For instance, i.c.v. administration of melanocortins induces hyperalgesia in rats (Sandman and Kastin, 1981), while central MC4R antagonism blocks pain-responses in hyperalgesia and potentiates the analgesic effects of morphine (Hellard et al., 2016; Kalange et al., 2007). The pharmacological studies on the melanocortin system have made the idea of MC4Rs as general mediators of negative valence intriguing.

The MC4Rs have been found on striatal D1R and D2R MSNs (Ophuis et al., 2014; Cui et al., 2012; Lim et al., 2012). The MC4Rs expressed by direct pathway D1R MSNs, but not indirect pathway D2R MSNs, were uncovered to mediate synaptic plasticity leading to stress-induced anhedonia (Lim et al., 2012), while having only minor effects on body weight and basal food intake (Cui et al., 2012; Cui and Lutter, 2013). Surprisingly, the subpopulation of MC4Rs on D1R neurons has been demonstrated to play a role in procedural memory (Cui et al., 2012). It is worthwhile noting that the experiments conducted in this study were based on negative reinforcement (watermaze) or self-administration of a high-fat diet over several days. Therefore, it is difficult to determine if the observed effects indeed were memory related, or perhaps rather a consequence of changed motivational valence and metabolic side effects. Evidence exist that the MC4R knockout mice when fed a high-fat diet over several consecutive days, as in the self-administration paradigm, eventually decrease their food intake compared to wildtype controls (Panaro and Cone et al., 2013). This could explain the positive phenotype occurring in the procedural learning paradigms. 


\section{ACETYLCHOLINE IN MOTIVATION AND ADDICTION}

\section{Acetylcholinergic circuitry}

Cholinergic neurons form a wide network throughout the CNS. Acetylcholine signaling in forebrain structures has long been implied in attention, learning and memory. The principal source of acetylcholine to the cerebral cortex and hippocampus is the basal forebrain complex. But other cholinergic neurons (nucleus basalis of Meynert, the horizontal nucleus of the diagonal band of Broca and the magnocellular preoptic area) project to the neocortex, olfactory bulb, amygdala and limbic cortex (Brady et al., 2012). Modern studies, utilizing optogenetics to investigate the role of the forebrain acetylcholine projections, have confirmed the previous views on the role of acetylcholine in cognitive functions (Hersman et al., 2017; Jiang et al., 2016; Zant et al., 2016; Pinto et al., 2013). Interestingly, the brain also has specific cholinergic neuropopulations innervating motivational brain areas such as NAc and VTA. These include acetylcholinergic neurons that can modulate either local or distal neuro-circuitry. The mesolimbic system is innervated by cholinergic projections arising from two brainstem nuclei: The laterodorsal tegmental (LDT) nucleus and the pedunculopontine (PPT) nucleus (Oakman et al., 1995; Dautan et al., 2014), while the striatum has its own population of cholinergic interneurons, which constitutes overall 1-2\% of its neurons (Witten et al., 2010).

The cholinergic interneurons of the NAc have been found to exert tight control over proximal neurocircuitry. For instance, optogenetic activation of cholinergic interneurons in the NAc was shown to enhance local phasic dopamine release (Cachope et al., 2012) and regulate MSN activity (Witten et al., 2010). The cholinergic interneurons are characterized by their tonic activity. They exhibit continuous spontaneous action potentials with a frequency of 2-8 Hz. The multiplicity of cholinergic neurons in the brain underlines the importance of this system, but also complicates the dissection of its functionality.

\section{Acetylcholine and reinforcement learning}

Acetylcholine has been suggested to play a role in reinforcement learning to both natural reward and drugs of abuse. The distinct firing-pattern of striatal cholinergic interneurons may encode a functional component related to reward learning, as behavioral studies in primates have shown that the interneurons pause their activity during display of a conditioned stimulus predicting reward (Joshua et al., 2008; Aosaki et al., 1994). In contrast to these observations, it has been demonstrated that rats display a significant increase of NAc acetycholine during the learning of a drug of abuse operant runway model for morphine, remifentanil and cocaine (Crespo et al., 2006; Crespo et al., 2008). Findings on cortical acetylcholine in the 5-choice- 
serial-reaction-time-task (5CSRTT) mirror those of the striatum during the operant runway-procedure. Dalley and colleagues demonstrated that acetylcholine levels in the cortex of rats increased specifically during contingent performance in the 5CSRTT task (Dalley et al., 2001). Hence increased synaptic acetylcholine, in striatal and cortical circuitry, is associated with the learning of a reward-associatedtask and not only the reward consumption. According to this notion, optogenetic inhibition of the NAc cholinergic interneurons attenuates the acquisition of cocaine conditioned place preference (Witten et al., 2010). Yet, the question remains: What does the pause in striatal cholinergic interneuron activity in response to rewardpredicting cues signify? Perhaps the source for synaptic acetylcholine during operant responding is not the cholinergic interneurons, but rather the projection-neurons in LDT or PPT?

There exist several contradictory studies on the role of acetylcholine in rewardlearning, with results ranging from U-shaped dose-response curves towards cholinergic receptor agonists, to opposing results from lesion and inhibition studies (as reviewed by Grasing, 2016). The overall picture shows how the influence of acetylcholine on reinforcement learning follows a bell-shaped curve, where too little or too much transmitter in the synapse is unfavorable (Grasing, 2016). This is not a new concept, as it is known that acetylcholinesterase inhibitors (which directly elevate the level of acetylcholine) only exert their effect at lower doses, whereas higher concentrations impair learning (Braida et al., 1996; Braida et al., 1997).

In this context, it is not too surprising that newer studies have revealed striatal acetylcholine to have a modulatory role rather than directly reinforcing properties. Neither optogenetic activation nor inhibition of choline acetyltransferase (ChAT)positive neurons in the NAc is capable of inducing place preference or avoidance (Lee et al., 2016).

\section{The muscarinic acetylcholine receptor M4}

The complexity of cholinergic signaling may also involve differences in expression of acetylcholinergic receptors on diverse subsets of neurons. In this manner, specific types of neurons carrying the same types of receptors, or the same type of neurons carrying diverse receptors, could mediate maintenance of the appropriate acetylcholinergic (bell-shaped) effect-balance for reward perception and learning. Acetylcholine binds to two types of receptor-families, ionotropic and metabotropic, categorized according to their selectivity towards specific pharmacological ligands: The nicotinergic and the muscarinic acetylcholine receptors. Muscarinic receptors are GPCRs and exist in five subtypes. The M1, M3 and M5 receptors are excitatory (Gq) receptors, whereas M2 and M4 acetylcholinergic receptors (Gi) inhibit neuronal firing through decreasing the intracellular concentration of cAMP (Langmead et al., 
2008) and activating G-protein coupled inward rectifying potassium channels (GIRKs). Muscarinic M4-receptors (M4R) mediate opening of GIRKS via the G $\beta \gamma$ subunit, which leads to direct efflux of potassium ions from the cytoplasm causing hyperpolarization (Wess et al., 2007). The M4Rs in particular control synaptic acetylcholine tone in the striatum and VTA, as they provide feedback-inhibition of transmitter release from the presynaptic cholinergic neurons (Zhang et al., 2002; Tzavara et al., 2004). The M4Rs are highly expressed throughout the forebrain, with the highest expression-levels occurring in the striatum as shown with in-situ hybridization (Allen Brain Atlas, http://mouse.brain-map.org/experiment/show/261). Here they have been found to co-localize especially with D1R-expressing MSNs (Jeon et al., 2010). This particular location could make the M4Rs functional brakes on the striatal direct pathway. In this context, M4Rs have previously been shown to play a role in the responding to drugs of abuse. Studies have uncovered that global deletion of muscarinic M4 receptors leads to enhanced cocaine and alcohol selfadministration in mice (Schmidt et al. 2011; De la Cour et al., 2015). Moreover, the conditional deletion of M4 receptors from D1-receptor expressing neurons leads to increased locomotor responses to psychostimulants, as well as an elevated dopamine efflux in the striatum upon amphetamine injections (Jeon et al., 2010). A recent study on genetic polymorphisms within the M4R gene revealed a link to both cocaine and heroin addiction in human subjects (Levran et al., 2016). 


\section{AIMS}

The general aim of this doctoral thesis is to investigate how specific molecular pathways shape the understanding of good and bad, as well as motivation to seek and avoid such stimuli. Specifically, the thesis aims were:

- To elucidate the molecular mechanisms behind inflammation induced aversion and how immune signaling affects the mesolimbic system (Study I and II). In more detail, these studies aimed to explore the role of the following targets for the development of systemic inflammation induced aversion.

- Peripheral cytokines TNF $\alpha$, IL-1 $1 \beta$ and IFN $\gamma$

- PGE2- synthesis and signaling to PGE2 receptors

- Motivational circuitry - including striatal signaling and midbrain dopamine

- To investigate the role of melanocortin 4 receptors and their natural agonist $\alpha$-melanocyte-stimulating hormone in various types of aversion, as well as how this satiety-peptidergic system influences motivational neurocircuitry (Study III).

- To assess how different subpopulations of muscarinic M4 receptors expressed specifically on MSNs or acetylcholinergic neurons affect positive reinforcement behaviors and impulsivity (Study IV). 


\section{METHODOLOGICAL CONSIDERATIONS}

In the following section, methods and animal models employed for the thesis work will be clarified. The rationale behind the use of each technique and model will be explained, with a particular emphasis on design, generalizability and proper controls for exclusion of confounding variables. This section will focus on the experimental techniques where I have been directly involved in both design and conduct. Any remaining technical information can be found in the specific manuscript on the individual study.

\section{Experimental animals - The mouse}

In this thesis mice were used as the experimental model organism. Mice share a high level of biological equivalence with human beings, corresponding to $90 \%$ of their DNA being homologous with ours (Waterston et al., 2002). In this perspective, it is not surprising that biological foundations for affective state to a large extent are similar between mice and human beings. To give a few examples of this: The mesolimbic dopamine system encodes reward and motivation in the same way in mice as in primates and humans (Ramchandani et al., 2011; Eisenberger et al., 2010; Martinez et al., 2010; Volkow et al., 2007; Shultz and Romo, 1990), and structures such as the nucleus accumbens, hypothalamic nuclei, central and basolateral amygdala and medial prefrontal cortex ( $\mathrm{mPFC}$ ) are heavily involved in regulating affective state in both mice and humans (Tye and Deisseroth, 2012). Currently the mouse is the most preferred animal model in neuroscience. The reason for this is not only the high level of biological homology with humans, but rather that mice are extremely suited for genetic engineering, enabling various kinds of target-specific manipulations. Null mutations can be introduced in virtually all genes of the mouse by applying the mechanism of homologous recombination in embryonic stem cells via gene-targeting techniques (Copeland et al., 2001). Mouse embryonic cells by nature have a higher frequency of homologous recombination than any other cultured cells. This fact, in combination with the ability to generate fertile mice from embryonic stem cells in culture, makes the mouse extremely suited for genetic engineering (Gardner and Brook, 1997; Glaser et al., 2005). Collectively the abovementioned features make mice appropriate experimental animals in the search for new treatment targets for human psychopathologies. Still an important problem in preclinical research remains: Finding suitable behavioral models, with a high level of face-validity and translatability, for exploring affective state in mice. 


\section{Ubiquitous knockout mice}

In the 1980 's, it was discovered that microinjections of foreign DNA into the cell nucleus of embryonic stem cells lead to genomic integration by homologous recombination in mice. This breakthrough allowed for an array of different modifications to be introduced to the mouse genome (Thomas and Capecchi, 1987; Doetschman et al., 1987). In this manner, scientists could take control over the expression of specific genes and the first knockout mice were produced in 1989 (Thompson et al., 1989; Koller et al., 1989; Zijlstra et al., 1989). These were ubiquitous knockout mice, which meant that they lacked a specifically chosen gene from their genome in every cell of the body. In study I, II and III ubiquitous knockout mice were employed for studying specific components of immune-to-brain signaling and the role of MC4Rs in motivational valence to negative stimuli. These mice had null-mutations targeting the following proteins: Caspase-1, IL-1R, TNF $\alpha$ R1/2, TNF $\alpha$ R1 and IL-1R, COX1, COX2, mPGES1, EP2R, EP3R, IFN $\gamma$, IFN $\gamma \mathrm{R}, \mathrm{Cxcl10}$, Cxcr3. EP1R and MC4R knockout mice are also ubiquitous, but were generated using a different strategy than that of classical null mutation mice. These are conditional mutagenesis mice as specified in the section below. When working with global knockout mice, considerations should be made on viability, as well as on developmental and compensatory mechanisms. Several cellular receptors involved in immunity are also key players in embryonic and neonatal development (Turgeon and Meloche, 2009). For instance, complete abolishment of the PGE2 EP4receptor gene (ptger4), which is a key player in adult immune signaling, is associated with neonatal heart and respiratory failure (Nguyen et al., 1997). Furthermore, viable knockout mice may develop compensatory adaptations or other secondary effects due to absence of the explicit gene. This complicates interpretation of results arising from global null mutations, and may make translation for development of new treatments difficult. The global nature of the null mutations in classical knockout mice may also obstruct the uncovering of discrete biological mechanisms, as several biological sites are affected. Due to these limitations, complementary interventions and biological measurements are pivotal for ensuring appropriate deductions when employing ubiquitous transgenic mice. In the present studies, pharmacological manipulations combined with measurements of gene expression and specific metabolites in restricted brain-regions have been used as complementary tools for evaluating results arising from null mutations. 


\section{Conditional strategies for knockout and knockin}

Site-specific recombination and gene targeting in murine embryonic stem cells, as described above, has resulted in the development of technologies that overcome some of the issues encountered with ubiquitous null mutations. Conditional mutagenesis technologies enable induction of mutations, which are specified according to cell-type and can be temporally regulated. A multitude of conditional knockout and knockin mice have been generated based on conditional mutagenesis, numerous of which are commercially available. In the studies of this thesis several transgenic mouse-lines have been used in order to investigate specific molecular pathways. The most common type of conditional mutagenesis utilizes DNAprocessing enzymes from other organisms, such as prokaryotes and lower eukaryotes, named recombinases. Recombinases mediate DNA rearrangements by breaking and joining the DNA-molecules at specific sites (recombination targets). So far, only three of these recombinases have been shown to function efficiently in embryonic stem cells when introduced artificially, of which the Cre-recombinase is the most widely used. The $E$. coli $\mathrm{P} 1$ phage Cre-recombinase specifically recognizes short DNA-sequences named loxP-sites (Sternberg et al., 1986; Branda and Dymecki, 2004). LoxP sites consist of 34 base pairs (bp), which include two $13 \mathrm{bp}$ palindromic sequences, separated by an 8 bp spacer-sequence (Branda and Dymecki, 2004). The Cre-recombination system can be used for generating conditional knockout mice by placing the Cre-gene under control of a cell-type specific promoter, while expressing the loxP-sites surrounding the gene of interest ubiquitously. This will cause inactivation of the gene of interest to occur only in cells where the promoter controlling the Cre-gene is active. For this kind of application, two transgenic mouse-lines are generated. In one mouse line, two loxP-sites are inserted on each side of an essential exon of the target-gene by homologous recombination, thereby producing a floxed ("flanked by lox-P sites") mouse (Branda and Dymecki, 2004; Lodish et al., 2008). The loxP-sites are introns and will not disturb the normal function of the target-gene. Another mouse line, the Cre-mouse, carries the introduced Cre-gene linked to a cell-type specific promoter, as described above. The Cre will not affect the function of other genes when placed appropriately in the DNA-sequence, due to its specificity towards the bacteriophage loxP-sites. When the two mouse-lines are crossed, they will produce Cre-loxP mice resulting in cell-specific recombination according to the chosen Cre-driving promoter. Hence the Cre-expressing cells are the only cells where recombination catalyzed by Cre occurs, leading to deletion of the target-gene located between the two loxP-sites (Branda and Dymecki, 2004; Lodish et al., 2008). 
A

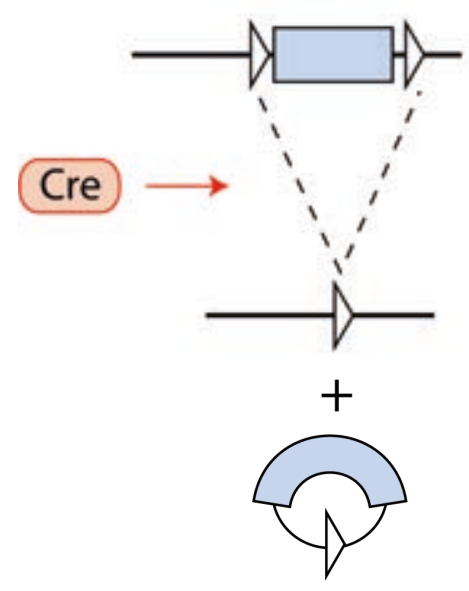

B

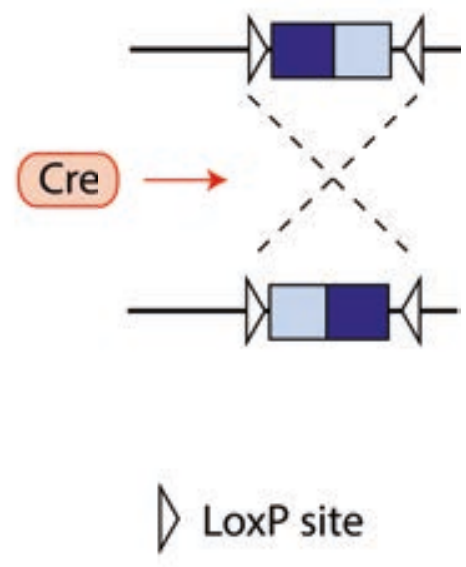

Figure 5. A schematic representation of Cre-mediated recombination via loxP-sites.

The Cre-recombinase is placed under control of a specific promoter. When Cre is expressed in the same cell as the DNA-sequence flanked by loxP-sites, it will either mediate $(A)$ excision of the gene if the loxP-sites are oriented in the same direction or $(B)$ inversion of the target gene if they are in opposite direction.

Dependent on the orientation of the lox-P sites, in the same direction or opposite to each other, Cre-recombinase will mediate either excision or inversion of the DNAsequence they flank (Branda and Dymecki, 2004), as illustrated in figure 5. This feature has allowed for the development of the double-floxed inverted open reading frame (DIO). Introduction of mutations in the 8 bp spacer-regions of wildtype loxPsites has generated artificial heterospecific loxP-sites. This is relevant, as Cremediated recombination is inefficient between two loxP-sites differing in spacerregions (Hoess, Wierzbicki and Abremski, 1986; Lee and Saito, 1998). In this manner, the DIO-technique utilizes two different sets of heterospecific, antiparallel loxP-type recombination sites (loxP and lox2272), which in the presence of Cre induces inversion of the coding sequence followed by the excision of two of the loxP-sites. This process leaves one loxP and one lox 2272 recombination site in opposite orientation, and therefore unable to be further recombined (Schnütgen et al., 2003; Atasoy et al., 2008; Branda and Dymecki, 2004) as illustrated in figure 6 on the next page. The DIO has become one of the most commonly used Cre-loxP based systems for introducing expression of genetic-constructs in transgenic mice, and is particularly employed in viral vector-based strategies, which were used in study I and III (Atasoy et al., 2008; Urban and Roth, 2015). 


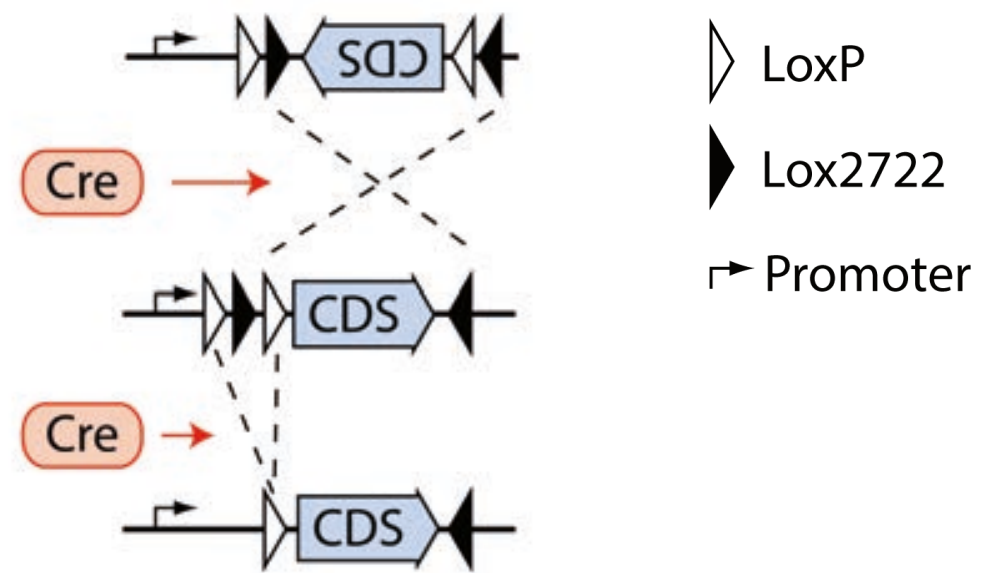

Figure 6. A schematic representation of the double-floxed inverted open reading frame (DIO). The Cre-recombinase is placed under control of a specific promoter. When Cre is expressed in the same cell as the DNA-sequence flanked by loxP-sites, it will first recognize the lox2722-sites and mediate inversion. The gene is now placed in the RNA polymerase reading direction and is functional. Subsequently the Cre will mediated excision of the wildtype loxP sites, preventing further recombination as only a loxP and lox2722 remains.

Another interesting application of the Cre-loxP system is that of re-expression of genes of interest in already ubiquitous knockout mice. Disrupting the expression of a target-gene, by inserting a non-coding-sequence called a "stop-cassette", is typically used for generating ubiquitous knockout-mice. If the stop-cassette is flanked by loxP-sites, the knocked-out gene can be reintroduced by removal of the stop-cassette via Cre-recombination, following the same principle as explained above (Branda and Dymecki, 2004). This mechanism was utilized for generating global EP1R and MC4R knockout mice, and subsequently rescuing their expression on specific neural populations by using D1R-CreERT2 or DAT-CreERT2 lines in study I and III. Knock-in strategies strengthen the conclusions derived from studies employing transgenic interference of specific target-genes. The re-expression of genes provides direct evidence of the necessity of that specific gene for its associated phenotype, whereas in the case of knockout observed consequences may be due to adaptive or secondary effects. Finally, the Cre-recombinase can be controlled temporally, thereby preventing developmental adaptive problems arising from gene knockout. In the present work two means for temporal induction of the Cre-recombinase were utilized: Ligand-dependent inducible Cre-recombinase mouse-lines and virus equipped with a Cre-recombinase-carrying construct. By adding a mutant estrogen- 
receptor ligand-binding domain to the C-terminus of the Cre-recombinase, it is possible to control translocation of Cre to the cell nucleus, and hence regulate its ability to approach lox-P sites and excise the target-gene. In this manner, the recombination requires ligation of the modified estrogen-receptor by 4-OHTamoxifen. We have utilized transgenic mice based on the CreERT2-system, which is the most sensitive for inducing both translocation and recombinase activity (Indra et al., 1999). The following ERT2-lines were used in study I, II and III: Slco1cCreERT2, DATCreERT2 and Drd1CreERT2. To ensure that off-target Tamoxifeneffects do not influence behavioral readout, control animals (mice carrying the floxed alleles, but no Cre) underwent the same Tamoxifen administration protocol as the test-animals (mice with floxed alleles carrying the Cre-recombinase gene).

Though the above-mentioned techniques for cell-specific recombination have provided more exact means for elucidating molecular mechanisms than the ubiquitous null mutations, each transgenic Cre-loxP mouse should be carefully evaluated. The use of Cre-dependent reporter-genes (e.g. eGFP, tdTomato) has allowed for target-site recombination to be properly validated. Yet, considerations should be made when choosing an appropriate promoter for expression of the Crerecombinase, as several cells-types express similar proteins and thereby utilize the same promoter-sequences. In study II, unintended knockout of IFN $\gamma \mathrm{R}$ using the Slco1c-CreERT2 line was present in the spleen. It is unlikely that the spleen endothelial cells are directly involved in immune signaling to the brain, but its potential contribution to inflammatory aversion signaling cannot be excluded based on these experiments. Lack of expression in other cells than the hypothesized targetcells, could be a confounding variable preventing scientists from properly validating their hypothesis. Such limitations of the Cre-loxP system should be considered when drawing conclusions based on results from the technique. Furthermore, when using any kind of transgenic animals, whether conditional or ubiquitous, it is important to exclude potential confounding factors arising due to changed health status or baseline behaviors (locomotor responses, metabolism, learning etc.). The behavioral aspects monitored in the experimental paradigms, should be considered regarding the overall phenotype of the animals - that is: Are the mice capable of performing the task?

The transgenic lines utilized in the current studies have been thoroughly described in the literature and by the commercial providers (please see references in the individual studies). In study I, III and IV, the most central transgenic animals were investigated to ensure intact learning and responding to alternative rewarding or aversive stimuli in the experimental conditioning paradigms. 


\section{Stereotaxic surgeries}

The use of stereotaxic surgeries in preclinical neuroscience has allowed for the direct manipulation of specific cell populations in brain areas of living animals. In study I and III, stereotaxic surgeries for the purpose of specific microcannula placement or viral vector injections were employed.

Briefly, animals are anesthetized using isoflurane (1.0 - 1.5\%) and when deeply asleep, they are mounted on a stereotaxic frame. The stereotaxic frame ensures proper fixation of the head of the mouse for the surgery, and provides precise placement of the surgical tools according to the target brain area (syringe, cannulaholder etc.). Species-specific stereotaxic atlases specify 3D coordinates of brainareas in regard to anatomical structures on the skull (lambda and bregma). A microdrill is used for making tiny holes in the skull through which the dura can be penetrated, and subsequently a syringe or cannula can be lowered into the brain for injection of viral vectors or specific placement. In this way, the stereotaxic surgery technique allows for exact and direct targeting of various brain areas. The animals are treated with antiseptics (chlorhexidine), maintained on a heating-pad, and surgery wounds are enclosed or sutured. When given postoperative analgesia for 48 hours, mice recover this type of surgeries exceptionally well, without signs of pain or behavioral changes. In animals that received a microcannulation surgery, the cannula is mounted to the skull with anchor-screws and dental cement, and a dummy-probe is placed in the cannula to protect it from contamination and blockage. Dummy-probe insertion does not cause pain or discomfort as it is bypassing skin, muscle and meningeal tissue through the guide cannula (Geiger et al., 2008). Issues with stereotaxic surgeries include not only problems related to anesthesia and painrecovery, but also to the destruction of brain-tissue on the path of reaching the targetarea. Destruction of brain-tissue is minor when using thin needles, with a large Gauge, for viral injections. However, in the case of microcannula (which are $0.4 \mathrm{~mm}$ in diameter) the effect on bypassed brain-areas, in the small mouse-brain, might be detrimental. To ensure that consequences from surgical procedures do not become confounding variables in the final experimental design, control animals go through the same surgeries as test-animals.

\section{Viral expression strategies}

In study I and III, viral-vector transduction using adeno associated viruses (AAVs) were employed for transgene expression-strategies. This type of genetic tools was enabled by the identification of viruses with the potential to be engineered into effective gene-delivery (transduction) vectors. AAVs allow temporal and spatial control over expression of transgenes, and therefore have been used for a broad array of applications in preclinical neuroscience. 
Compared to other viral vectors, the parvovirus AAVs have low levels of immune reactivity, stable transduction and are easy to work with, as they are considered nonpathogenic. The AAVs can carry constructs of sizes up to approximately 4.7-5.0 kilobases, corresponding to the size of the wild-type AAV genome (Aschauer et al., 2013). This has made it possible to make cells in the brain express various kinds of transgene proteins, such as "Designer receptors exclusively activated by designer drugs (DREADDs)" (please see the subsequent section on chemogenetics) and Crerecombinases in combination with fluorescent reporter proteins (e.g. mCherry, eGFP). Several considerations on choice of experimental protocol, in terms of AAVserotype, injection-dose, type of promotor for transgene expression and expression time, should be made prior to experimentation.

Natural AAV serotypes have been described based on specific surface-properties of the capsid (the protein-shell of the virus). Recombinant AAV-vector serotypes have arisen from providing AAV2 with the cap-genes of other AAV-serotypes, thereby changing the characteristics of AAV2 in its surface-interaction with target-cells (Zincarelli et al., 2008). Significant differences have been reported between serotypes of AAVs in transduction-efficacy of specific tissue and cell types. Hence it is important to select the appropriate AAV-serotype dependent on the particular application. In the current work AAVs were employed solely for the transduction of neurons, but were applied in different areas of the mouse brain: $\mathrm{CPu}$ and VTA (study I); VTA, whole striatum, arcuate nucleus and NAc shell (study III). AAV5 and AAV8 have been demonstrated in a multitude of studies to effectively transduce neurons of these brain areas (Aschauer et al., 2013; Atasoy et al., 2012; Betley et al., 2015; Wang et al., 2015; Witten et al., 2010). Another important aspect to consider for experimental design is that both AAV5 and AAV8-serotypes are capable of axonal transport in both retro- and anterograde directions (Castle et al., 2014). This phenomenon, can be regulated in a dose-dependent manner. For instance, by using lower doses of AAVs for transduction, axonal transport can be diminished (Castle et al., 2014). In study III, a high dose of AAV8 (600 nl per site of $3 \times 10^{12}$ genome copies $/ \mathrm{ml}$ ) was applied for retrograde transduction from the NAc shell to the ARC, compared to direct targeting of POMC-somas in the ARC ( $250 \mathrm{nl}$ per site of $3 \times 10^{12}$ genome copies $/ \mathrm{ml}$ ). The axonal-transport features of AAVs emphasize the importance of transduction-restriction to ensure proper target-selectivity. This can be achieved by control over gene-expression via the Cre-loxP system, as described previously. Expression of the viral-vector proteins can be made Cre-dependent by utilizing either a stop-cassette or a DIO, as described in the previous section on "conditional strategies for knockout and knockin". In study I, transduction with GqDREADDs and mCherry was done in the DATCreERT2 mouse-line to ensure dopamine neuron specificity, and in study III the POMCCre mouse-line was 
employed for both local and anterograde transduction of Arcuate nucleus POMCneurons. Another way of restricting viral transgene expression is by selecting an appropriate promoter, compatible with the target cell-type, for driving the expression of the transgene. For instance, the human synapsin 1 gene promoter (hSyn) confers highly neuron-specific transgene expression from AAV-vectors (Kügler et al., 2003). hSyn is commonly used as a promoter in commercially available AAV-vectors. The vectors used in the present studies were all equipped with a hSyn-promoter and were either purchased from University of North Carolina (UNC) vector core, or kindly provided by professor Johan Jakobsson at Lund University. In study I and IV, the hSyn promoter defined AAV5 cell-transduction for expression of Cre-recombinase. In the case of Cre-recombinase carrying constructs, the Cre-loxP system selfevidently cannot be utilized for selective expression of the construct. AAVtransduced transgenes typically exhibit stable expression-levels in the brain three weeks after injection (Atasoy et al., 2012; Betley et al., 2015; Wang et al., 2015; Witten et al., 2010). Hence it is important to time the start of behavioral- or imaging experiments with the stereotaxic injections of the viral vectors, i.e. 3 weeks later.

\section{Chemogenetics}

The term chemogenetics covers the bioengineering of proteins to interact with previously unrecognized molecular substrates. Study I and III include investigations of neurocircuitry employing DREADDs. Various G-protein coupled receptors (GPCRs) have been modified in such a manner that they no-longer respond to natural ligands but instead are specifically activated by artificial designer-drugs. The Bryan Roth group was the first to successfully modify the human muscarinic M3 and M4 receptors for this purpose using site-directed mutagenesis in yeast. They discovered two specific point-mutations that made it possible to generate muscarinic receptors with nanomolar potency to the biologically inactive clozapine analog, clozapine- $\mathrm{N}$ oxide (CNO). These modified receptors were insensitive to the endogenous receptor ligand acetylcholine, while maintaining low levels of constitutive activity. The Roth laboratory has generated several types of DREADDs, where the two most popular are the hM4Di and hM3Dq, which are Gi and Gq-coupled respectively (Urban and Roth, 2015). Several transgenic strategies for cell-type specific expression of DREADDs have now been developed based on the Cre-loxP system. In the present work AAV8-dependet transduction with commercially bought vectors carrying DREADD-constructs have been utilized in either a Cre-dependent (DIO) or Creindependent manner. In this way, neuronal firing can be induced using $\mathrm{CNO}$ mediated hM3DGq-activation or silenced using hM4DGi-activation. While hM4DGi-receptors have been shown to activate G-protein coupled inward-rectifying potassium channels (GIRKs) leading to hyperpolarization, the molecular 
mechanisms behind hM3DGq induced neuronal firing involves increased intracellular calcium levels, but are otherwise not clarified (Roth, 2016).

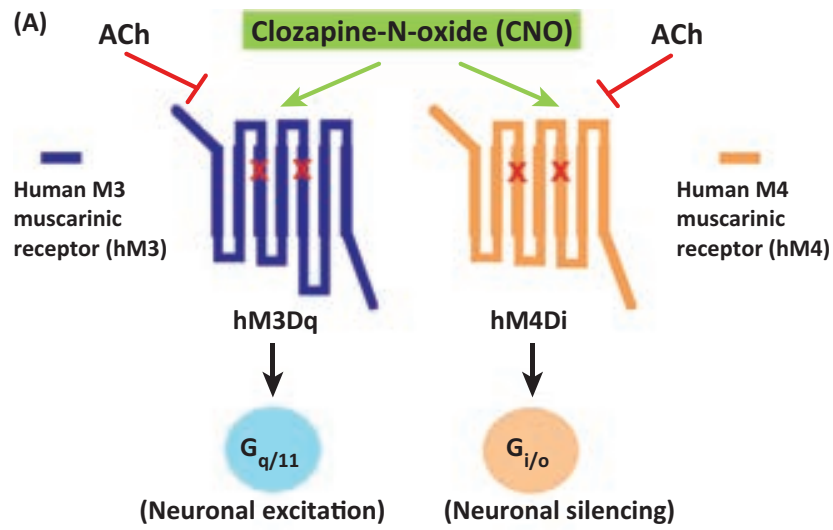

Figure 7. A schematic representation of the modified muscarinic receptors used for chemogenetics, i.e. activating and inhibitory DREADDs: hM3Dq and hM4Di.

Original figure by Wess, Nakajima and Jain, 2013. Reprinted with permission from Elsevier

A drawback with DREADD-induced activation or inhibition is that the designer drug CNO is metabolized back to clozapine, though this occurs at a very low rate (Roth, 2016). Recently the question has been raised if DREADDs indeed are activated by $\mathrm{CNO}$ or rather by the clozapine-metabolites, as CNO does not easily traverse the BBB (Gomez et al., 2017). These findings emphasize the importance of applying proper controls against potential off-target effects. Hence, all control-groups receive the same doses of CNO as the test-groups (study I and III). The only difference between test and control groups is that control group animals do not express Crerecombinase. These animals will as a consequence not express the DREADDconstructs and therefore only experience DREADD-independent CNO-effects. Studies have shown that DREADD-activation effects on neural activity occur 10-15 minutes after i.p. CNO administration in vivo and persist for hours (Alexander et al., 2009). These ongoing effects make DREADDS particularly suited for conditioning paradigms with longer training sessions, where ongoing activation or inhibition of neural circuitry is desired. Proper timing of the i.p. CNO administration with cueconditioning is absolutely pivotal for the success of such experiments, as described in the Pavlovian conditioning on the next page: The strength of conditioning is dependent on the time-interval between presentation of the conditioned stimulus (CS) and unconditioned stimulus (US). For instance, if the US is CNO-induced hM3Dq-activation of specific neurons leading to an aversive state, it is absolutely 
pivotal that the animal experience the onset of $\mathrm{CNO}$ activation when exposed to the visual cues in the conditioning chamber and that this occurs within a relatively short time.

\section{Pavlovian conditioning}

Ivan Petrovich Pavlov was a Russian physician working on the physiology of digestion, when he discovered that his experimental dogs would salivate whenever the technician who would feed them entered the room, even in the absence of food. He concluded that the dogs through a learning process had come to associate the technician with food. Through the repeated representation of the technician (the neutral stimulus) with the food (the unconditioned stimulus), the technician had become a conditioned stimulus predicting the presence of food. In this very simple manner Pavlov described how through conditioning previous neutral stimuli can become salient, and predict the arrival of either pleasant or unpleasant stimuli. Pavlovian conditioning is a type of associative learning common to both animals and human beings. This type of learning follows the reward-prediction error and is dependent on dopamine, as explained previously in the section on reward prediction error of the introduction and illustrated in Figure 1. It occurs, as described by the example above, through the repeated pairing of a conditioned stimulus (CS) and an unconditioned stimulus (US). When the association has been formed between the two, the CS will elicit the conditioned response (CR). In the above example the CR was the salivation of the dogs, which naturally (unconditioned) would be stimulated by the representation of food. Conditioning will occur if the US and CS are presented simultaneously, or if the CS precedes the US by a short interval. If the time between the CS and the US is too long, the association will not be formed. It is clear that this type of learning is essential for our ability to predict outcomes of situations, which is crucial when seeking positive stimuli important for our survival and avoiding negative ones (Bear, Connors and Paradiso, 2007).

Pavlovian learning paradigms for rodents are typically based on conditioning towards a specific place or environment. In such paradigms, the CS will usually be environmental cues, which after repeated parings with an US will elicit either approach or avoidance behavior, depending on the nature of the US. When using a US with either appetitive or aversive properties, this paradigm is referred to as conditioned place preference (CPP) or conditioned place aversion (CPA), respectively (Sanchis-Segura and Spanagel, 2006). In the present studies, a threechambered conditioning box, more specifically the Panlab Spatial Place Preference Box (Harvard Apparatus), was employed for both CPP and CPA. This box consists of two visually distinct chambers connected by a Plexiglas corridor. Specific visual cues (CS) are present in each chamber: The left compartment has walls with black 
dots on a white background, while the right compartment has grey stripes on a white background.
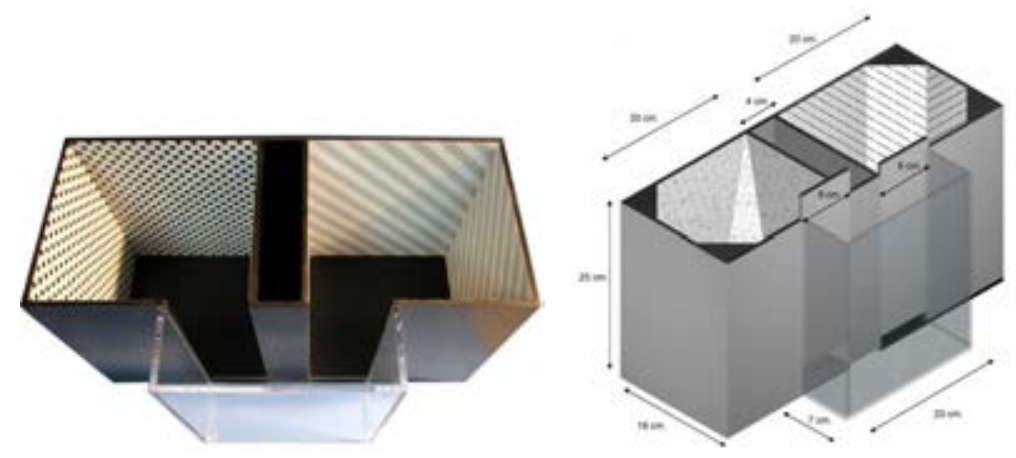

Figure 8. A picture and schematic representation of the Panlab Spatial Place Preference Box (Harvard Apparatus) with measures.

A so-called biased place conditioning procedure was used to measure aversion and preference using this box. In this biased procedure, we take into account that our place-conditioning box in its design has an innate tendency to generate preference towards one of the chambers. Most mice naturally prefer the dotted chamber, irrespective of box or experimenter. To overcome this bias, the US was paired with the non-preferred chamber when it was rewarding, whereas in the case that the US was aversive, it was administered in the most preferred chamber. This method has been shown to produce reliable conditioned place responses comparable with other paradigms (Bardo et al., 1995) and prevents already existing bias towards either of the chambers to interfere with the conditioning and thereby become a confounding variable. Place-conditioning paradigms have also been demonstrated to function for human reward learning in the same manner as for rodents. Studies have demonstrated that time spend in the conditioning room is proportional to the subjective feelings towards the US (Childs and de Wit, 2010). These findings have provided translational strength to the Pavlovian place-conditioning paradigm. Still, considerations should be made when using place-conditioning paradigms in animal models. For instance, it is important to set criterions ensuring proper choicebehavior, such as number of crossings between chambers, as a positive result in the place-preference box could be the consequence of inactivity. Another concern, for the interpretation of conditioning results, is the lack of separation between effects related to acquisition and expression. Acquisition is the acquiring of the behavior based on changed affective or motivational components, and expression is the remembering of the already acquired behavior. It is difficult to know whether the 
experimental interventions, in particular when employing transgenic animals, used in CPP and CPA influence affective state as intended or interfere with the memory of the affective state. Hence, for these kinds or experiments appropriate control measures are pivotal.

\section{Models of systemic inflammation}

Lipopolysaccharide (LPS) is a typical component of the outer membrane of gramnegative bacteria. This active fragment of endotoxin produced by E. coli bacteria has been used in several animal and human studies to produce systemic inflammation. When E. coli LPS is administered systemically, the innate immune system quickly responds by inducing symptoms of the acute phase response (Saper, Romanovsky and Scammell, 2012; Dantzer, 2001). Intraperitoneal (i.p.) injections of LPS are known to generate a pro-inflammatory cytokine profile similar to that observed in both humans and experimental rodents during infections, which is also characteristic for several chronic inflammatory diseases (Dantzer et al., 2001; Teeling et al., 2010; Biesmans et al., 2013; Neurath, 2014; Estevez et al., 2017). For the studies in this thesis (Study I, II and III), a low-grade systemic inflammation model was employed applying $10 \mu \mathrm{g} / \mathrm{kg}$ LPS i.p. from K-235 E. coli. At this dose, mice display mild symptoms of the sickness syndrome including elevated body temperature and anorexia (Nilsson et al., 2017a). Many animal models used for investigating inflammation-induced negative affective state are based on administrations of high doses of LPS (500-2500 $\mu \mathrm{g} / \mathrm{kg}$ ) (O'Conner et al., 2009; Biesmans et al., 2013). It is worthwhile noting that these higher concentrations of LPS may cause septic shock in mice, and therefore are dissimilar to the inflammation-levels occurring during common infections and chronic inflammatory diseases in human beings. Hence the low dose of LPS $(10 \mu \mathrm{g} / \mathrm{kg})$ i.p. may represent an advantage, as it is more like normal inflammatory conditions and is similar to that used in studies showing that endotoxins rapidly causes depressed mood in healthy human subjects (Reichenberg et al., 2001; Eisenberger et al., 2010). In order to ensure that the behavioral effects observed from i.p. LPS administration were generalizable to systemic conditions, and not exclusively related to inflammation in the peritoneal cavity, we compared conditioned place avoidance behavior elicited by i.p. injections to that caused by intravenous (i.v.) administration (study I).

Similar to LPS, a myriad of pharmacological studies has demonstrated that systemic treatment with recombinant IL-1 $\beta$ induces all the above-mentioned aspects of sickness behavior (Dantzer et al., 2008). A common feature of various kinds of inflammatory diseases is the upregulation of pro-inflammatory cytokines. In this regard, IL-1 $\beta$ administration i.p. represents a useful strategy for simulating more general aspects of systemic inflammation as this cytokine is a general feature of 
many types of diseases. In study I, we used a dose of IL-1 $\beta$ (600 ng) which previously has been shown to induce anorexia, HPA-axis activation and fever in mice (Nilsson et al., 2017b; Matsuwaki et al., 2014; Saha et al., 2005). Despite its relevant characteristics in initiating general inflammatory signaling, it should be noted that recombinant IL-1 $\beta$ is an artificial ligand in comparison to LPS, as it produces high but short-lived inflammation (Reimers et al., 1991).

A common consequence of systemic cytokine signaling is the production of PGE2 at specific physiological sites (Saper, Romanovsky and Scammell, 2012). To demonstrate and mimic the inflammatory signaling of PGE2 in specific brain areas related to aversion, microinjections of 200 picomole PGE2 were given intra NAc or $\mathrm{CPu}$. This dose of PGE2 is corresponding to cerebrospinal fluid levels capable of eliciting sickness symptoms (Nilsberth et al., 2009).

\section{Other models of aversion}

An advantage of the Pavlovian place-conditioning paradigm is that it is applicable for measuring aversive aspects of various kinds of treatments. Classical models of conditioned place aversion utilize diverse types of unconditioned stimuli for mimicking different aversive states, such as dysphoria and stress (i.p. injections of kappa-opioid receptor agonists), gastric distress (i.p. injections of Lithium Chloride), or pain (local subcutaneous formalin injections). In study III, this type of CPA models was utilized for investigating the role of MC4Rs in various aversive states. The specific agents employed as unconditioned stimuli and their doses are described below.

The endogenous kappa-opioid receptor (KOR) system has been demonstrated to encode the dysphoric component of stress (Land et al., 2008). Pharmacological activation of KORs is known to evoke anxiety and dysphoria in human subjects (Pfeiffer et al., 1986; Millan 1990), and similar behaviors in rodents (Ehrich et al., 2015; Land et al., 2008). Therefore, the selective KOR-agonist U50.488 was employed for inducing dysphoric aversion. This pharmacological agent is commonly used for generating CPA in mice at doses of $2.5 \mathrm{mg} / \mathrm{kg}$ (Ehrich 2015; Land et al., 2008; 2009).

One of the most reliable and frequently used aversive stimuli is lithium chloride $(\mathrm{LiCl})$. This toxin is known to induce gastric malaise and hypothermia (McCann et al., 1989; Cunningham and Niehus, 1993). Hypothermia in mice is considered the biological correlate to nausea (Ngampramuan et al., 2014), and elevating ambient temperature during conditioning counteracts LiCl-induced aversion (Cunningham and Niehus, 1993), illustrating how important this particular aspect is to LiCl's aversive properties. At doses around $130 \mathrm{mg} / \mathrm{kg}, \mathrm{LiCl}$ has been reported to induce robust CPAs (Tzschentke, 2007). 
Finally, local inflammation induced by subcutaneous (s.c.) injections of $2.5 \%$ formalin into the hind paw produces CPA and clear nocifensive behaviors (flinching, paw licking and lifting) (Johansen, Fields and Manning, 2001). This model has in early studies with human subjects been described to induce a short-lasting intense pain of sharp burning quality, which is followed by a long throbbing ache lasting 3060 minutes. Due to these qualities, it is widely used in preclinical research for investigating the affective component of pain (Bevan, Cools and Archer, 1989).

\section{Models of cocaine addiction}

Most models investigating drugs-of-abuse reinforcement intend to mimic the three phases of drug addiction: Binge intoxication, abstinence and relapse. Such behavioral paradigms are based on either contingent or non-contingent drugadministration. In contingent administration models, the animal chooses to perform a specific behavior that leads to the delivery of the drug of abuse (i.e. operant responding), while in non-contingent administration models the animal is passively given the drug of abuse (as for Pavlovian conditioning and locomotor sensitization). Studies employing both types of drug administration may represent an advantage, as both categories of learning are important for the psychopathology of addiction. In study IV, both Pavlovian and operant conditioning models were employed for investigating the role of muscarinic M4 receptors on specific neural populations in cocaine reinforcement. During Pavlovian conditioning, cocaine was administered to animals i.p. prior to placement in the conditioning chamber of the place preference box. In contrast, in the operant runway paradigm the mice have to run to the goalbox in order to receive an i.v. injection of cocaine through a long polyethylene tube, thereby leaving the animal undisturbed. The dose of cocaine, time-point of administration and duration of training-sessions are three pivotal factors determining the strength of drug-reinforcement learning. Studies using mice have shown that the magnitude and duration of retention of cocaine place preference is determined by the dose administered. Brabant, Quertemont and Tirelli (2005) found that the highest dose of 3 tested (4, 8 and $12 \mathrm{mg} / \mathrm{kg}$ i.p.) produced the strongest and most persistent place preference scores. Several studies using a cocaine dose of $15 \mathrm{mg} / \mathrm{kg}$ i.p have found robust reward learning effects in the CPP paradigm (Fritz et al., 2011; Klawonn et al., 2017), hence this dose was used as US in study III and IV.

In the same manner, a previous study on M4-D1RCre mice demonstrated doseresponse relationships in a self-administration paradigm with significant differences at a concentration of $0.3 \mathrm{mg} / \mathrm{kg}$ i.v (Schmidt et al., 2011). Hence, this specific concentration of cocaine was utilized for the operant runway paradigm in study IV. In terms of generalizability, it is very difficult to compare the doses used for experimental animals with those commonly consumed by human subjects. This is in 
part because the drug administration-routes are very different. Human addicts most commonly consume cocaine intranasal or by smoking it. Therefore, the basis for comparability between human and mouse cocaine-doses lies in the positive reinforcement associated with the dose of cocaine, where drug-seeking in animals typically is considered an equivalent to drug-craving.

The importance of timing of cocaine administration and duration of training sessions for successful conditioning experiments, is in part due to the principles of the "opponent process theory" mentioned in the section on "reward allostasis" in the introduction. The strong A-process associated with cocaine consumption will naturally be followed by an equally strong B-process. If the conditioning is not timed accurately, the unpleasant B-process could inadvertently become the US. This effect has been demonstrated in preclinical conditioning-models using cocaine (Pliakas et al., 2001; Ettenberg et al., 1999). Consequently, mice are most commonly placed in the conditioning chamber immediately after cocaine administration for a short timeinterval of approximately 10-20 minutes (Tzschentke, 2007). The duration of the place preference-conditioning sessions in the current work was 15 minutes.

\section{Palatable food}

Palatable food is a natural positive stimulus commonly used for investigating normal motivational responses in rodents during both Pavlovian and operant conditioning experiments. Place conditioned behaviors to natural rewards are considered conceptually different to those arising from drugs-of-abuse. Natural reward-based place preference scores are believed to represent the conditioning of the unconditioned search for the reward, rather than the affective component associated with its consumption (Spiteri, Le Pape and Ågmo, 2000). Hence place conditioning towards palatable food primarily illustrates the spatial learning to find reward and the motivational aspects associated with this search. Various kinds of palatable food have been utilized for self-administration, operant runway and place preference paradigms, including sucrose-water, sucrose pellets with added tastes, sweetened condensed milk and Kellogs fruit loops (Wakonigg et al., 2003; Panaro and Cone, 2013; Tzschentke, 2007). Prior to establishing the palatable-food enforced paradigms used in study III and IV, a set of pilot-experiments where conducted for monitoring approach-behavior and overnight consumption in wildtype mice to different types of palatable food (i.e. apple, honey-covered oats, strawberry marmalade, fruit-loops, marzipan and Nutella $\left.{ }^{\circledR}\right)$. Among $\mathrm{C} 57 \mathrm{bl} / 6$ mice the most popular choice was honeycovered oats (START!®) and Nutella ${ }^{\circledR}$. Conditioning mice to honey-covered oats in the CPP-paradigm turned out to be difficult. This is because the mice used the conditioning-session to hide the oats under walls and obstacles, instead of eating them. Since food-induced CPP has been shown to depend on the actual consumption, 
rather than cues associated with prior or later reward (Maes and Vossen, 1993), it is not surprising that the mice failed to develop place preference towards the oat-paired chamber. Another important aspect to consider when choosing a palatable food stimulus is how it is metabolized. Differences in the preference-response of animals, to high fat or high carbohydrate foods, may depend on genotype-related variations in metabolism. When investigating the MC4R KO mice (study III) this was a particularly important feature to consider, as these mice are obese and respond very differently to fatty or sucrose-rich rewards (Mul et al., 2012) than normal mice. In regard to the above-mentioned considerations, Nutella ${ }^{\circledR}$ was the best candidate for palatable food-reinforcement, as it is both fatty and sweet, can be delivered in very small quantities and is a creamy paste, which prevents mice from hiding it (they rapidly realize they have to consume it).

\section{Other behavioral paradigms \\ Operant runway}

Operant runway-based procedures have been used to study reinforcing effects of various kinds of positive stimuli. In study III and IV, the operant runway was employed for investigating motivational features of palatable food reward and cocaine seeking.

In 1938 the American psychologist Burrhus Fredric Skinner coined the term operant conditioning. While Pavlov had demonstrated that during classical conditioning the environment affects the subject by punishment or reward leading to reinforcement of specific behaviors, Skinner proposed that in nature it is the subject affecting the environment. During operant conditioning the subject operates on the environment, which in turn leads to reward or punishment that will further enforce or block certain behaviors. During reinforcement learning, the subject will form associations between this operation and a reward (positive reinforcement) or an avoided punishment (negative reinforcement) (Bear, Connors and Paradiso, 2007).

Theories describing the role of dopamine in operant goal-seeking behavior emphasize either conditions antecedent to the behavioral response (motivation) or the consequences of the behavioral response (reward). The operant runway paradigm was developed in the Ettenberg laboratory, in order to better dissociate the motivational and reinforcing processes in drug-seeking behavior (Ettenberg, 2009). The idea behind this paradigm is that the nature of the operandum (the run and search) allows for more clear investigations of the antecedent aspect of reward, compared to classical self-administration paradigms based on momentaneous lever pressing. The operant runway is basically a long corridor with a start-box, for confinement of the animal prior to the task, and a goal-box, where the animal will receive the reward upon entrance. The time required for an animal to cross the 
corridor of the runway is the index of the animal's motivation to seek the reward, and is inversely proportional to the strength of the specific stimulus.

A clear advantage of the operant runway, over self-administration paradigms, is that it is built on a natural operandum for rodents (i.e. search and run behavior). A drawback of this procedure is, in the same manner as with CPP, that the cocaineadministrations are confined to a specific number, which prevents measurements of compulsive ceiling effects on intake (such as break-point) (Sanchis-Segura and Spanagel, 2006). But in this way, the operant runway model may be a suited paradigm for "operant mirroring" of findings made in the Pavlovian place preference paradigm.

\section{Locomotor measurements}

Offline measurements of locomotor-activity were done in study I and IV, by utilizing contrast-based video tracking-programs (EthoVision XT from Noldus and SMART 3.0. from Panlab). Decreased locomotor activity is frequently used as readout for inflammation induced sickness behavior (Dantzer, 2001). In study I, LPS induced decreases in locomotion was monitored over one hour, to observe sickness behavior in a duration comparable to that of the LPS CPA conditioning sessions. Animals were pre-injected 10 min prior to locomotor-recordings, in the same manner as for CPA-training. During this time, the animal is confined to a small chamber in a grey locomotor box and the experimenter has left the room to prevent unnecessary stress. To ensure that the recordings are done consistently and that other factors (such as external noise or the time-of-day) do not influence the behavior of the animals, intervention and control mice are recorded simultaneously and their allocation to chambers is interchanged between each run. The presence of other mice in the box could influence behavioral readout, but having internal controls for each run has a high value for ensuring comparability between the trials. It should be noted that affective state and movement have been suggested to share a common neural circuitry in the dorsal striatum. This is not too surprising, as positive affective state often is associated with increased motion, whereas negative emotions can be accompanied by inactivity (Kravitz and Kreitzer, 2012). This phenomenon makes it difficult to clearly separate the sickness-syndrome induced decrease in locomotion from that occurring as a consequence of sickness induced negative affect.

In study IV, cocaine induced locomotor sensitization was explored. Sensitization in general refers to an increase in a response specifically associated with the recurrence of a stimulus. Animals that are repeatedly administered with the same dose of cocaine (e.g. $15 \mathrm{mg} / \mathrm{kg}$ ) will develop a significant increase in movement over the time-course of administrations (Sanchis-Segura and Spanagel, 2006). Even though locomotor sensitization is a behavior dissimilar from associative learning, studies 
have shown that a conditioned locomotor-component can occur. This is typically the case when the drug of abuse is administered in another environment than the homecage (Robinson and Berridge, 2003). It is important to clarify this component by monitoring locomotor activity of animals, which have undergone drug-induced locomotor sensitization, in the drug-paired environment in absence of the drug. This was done in study IV. Furthermore, the administration route is extremely important for the success of locomotor sensitization to cocaine. Administration routes with fast onset of drug-effects are particularly feasible for sensitization, such as i.p. injections (Sanchis-Segura and Spanagel, 2006). Yet, repeated i.p. administrations are physically stressful for mice, and need to be performed with extreme care and sensitivity, otherwise the animals will not increase their locomotor responses.

\section{Immunohistochemistry}

Antibodies are large proteins equipped with specific binding domains ( $\mathrm{Fc}$ fragments) for recognition of foreign molecules, so called "epitopes". An epitope may be any kind of biological structure, including specific sites of proteins. The antibodies have evolved as part of the vertebrate immune-system strategy for recognizing and evading various kinds of pathogens (Schroeder, 2015). Hence the adaptive immune system of vertebrate species will, when encountering foreign epitopes, generate antibodies with high specificity against these. This principle has been utilized for producing a multitude of antibodies for detection of various kinds of proteins in biomedical research (Saper and Sawchenko, 2003). Immunohistochemistry is a term that encompasses several antibody-methods used for identifying proteins in intact tissue. Many advances have been made to increase the sensitivity of the antibodysignal for microscopic visualization, the most important being the development of systems based on secondary antibody detection (Sternberger, 1979). In such systems, the specimen will be introduced to the primary antibody, which will bind to the target-epitope, and in turn be recognized by a species-specific secondary antibody, providing amplification of the primary antibody signal. In fluorescent immunohistochemistry the secondary antibody is conjugated with a fluorophore. As fluorophores have the ability to absorb light at one wavelength and emit them at a much longer wavelength, the emission and excitation light can be separated allowing for high-resolution visualization of the epitope. Fluorescent immunohistochemistry has made the detection of several epitopes simultaneously possible; this is in part due to the different emission spectra of potential conjugated fluorophores. In paraformaldehyde-fixated brain sections all biological structures remain intact, which permits investigation of complete neurons and their protein expressionpatterns. Fluorescent immunohistochemistry in combination with confocal microscopy was utilized to validate cellular specificity of recombination-strategies. 
In study I-III, Cre-dependent expression of fluorescent reporter-proteins (i.e. mCherry, tdTomato, eGFP) was introduced, by employing DIO or floxed stopcassette strategies, via viral-transduction or by using a transgenic reporter-line.

Several precautions have to be taken when employing antibodies for anatomic studies, the primary ones are concerning specificity. Most antibodies are characterized using western blotting to verify staining of protein-bands with equivalent weight as that expected of the epitope, but the gold standard for testing antibodies is to stain tissue with and without the epitope of interest (Saper and Sawchenko, 2003); This can be done by utilizing tissue-sections from knockout animals. In the present work, previously characterized antibodies were employed. These include anti-CD-31, anti-pp-enkephalin, anti-eGFP, anti-RFP, anti-Tyrosine Hydroxylase, anti-Lipocalin-2.

\section{Enzyme-linked Immunosorbent Assays}

Enzyme-linked Immunosorbent Assay (ELISA) is another common technique employing the principles of antibody epitope-recognition as described on the previous page.

These techniques are typically solid-phase methods, where the epitope-specific antibody is non-covalently bound to a solid phase-support in a microtiter-plate (Lequin 2005). Epitopes from sample-homogenates will bind to the antibodies, and subsequent washing of the plates will ensure specific containment of the epitope for detection. In many ELISAs (like the assays used in study I and III), detection is based on the principle of competitive-binding to the primary antibody between the sample epitope and an introduced tracer-carrying epitope. Since the concentration of the epitope-tracer is held constant in all wells, while the sample-epitope levels are variable; the amount of tracer-epitope that can bind to the antibody in the wells is inversely proportional to the concentration of sample-epitope already bound in the wells. The levels of tracer can subsequently be determined by spectrophotometry and the concentration of epitope calculated from these results (www.caymanchem.com/pdfs/514531.pdf). Due to the principle of competitive ELISAs, the accuracy of sampling, treatment of samples and pipetting is absolutely pivotal. This is because proper detection primarily relies on the concentration of metabolites and epitopes used in the assays. Many commercial ELISAs are now available for detecting metabolites in blood-samples and tissue-homogenates. The accuracy of these ELISAs is primarily determined by the quality of the applied antibodies. In many cases the ELISAs will recognize other molecules with similar structure as the epitope, leading to cross-reactivity. In study III, corticosterone levels were directly determined from heart-blood using a commercial ELISA kit. 
Direct determination of PGE2-levels is difficult to achieve, since PGE2 is rapidly metabolized in the body. Therefore, indirect measures have been developed based on ELISA-detection of PGE2-metabolites (13,14-dihydro-15-keto PGA2 and 13,14dihydro-15-keto PGE2) converted to a single stable derivative. A commercial PGE2metabolite ELISA was employed for detection in homogenates of specific brainareas (forebrain, $\mathrm{NAc}, \mathrm{CPu}$ ) in study $\mathbf{I}$.

\section{Electrophysiology and optogenetics}

The recognition that animal cells exhibit bioelectrical properties led to the development of sophisticated electrodes for recording such events. Glass micropipettes with tips in the size of tens of nanometers, filled with electrolyte-rich solution and a recording-electrode connected to an amplifier, have permitted recordings from virtually any type of neuron. Electrophysiological recordings can now be done from neurons in acutely prepared brain slices using patch-clamp recording techniques, including the cell-attached patch.

During such recordings, input through the patch-pipette-electrode is compared to that of the extra-cellular reference electrode. By using a current-clamp configuration, it is possible to measure action potentials across the cell membrane. This is because a fixed amount of current is injected through the micropipette to hold the current across the membrane constant (clamped). According to Ohm's law where $U=I^{*} R$ (U is voltage; $\mathrm{I}$ is current; and $\mathrm{R}$ is resistance across the membrane), keeping the current constant will allow for the recording of voltage-changes across the cell membrane. In this manner, the membrane potential is recorded as the voltage difference between the inside and the outside of the cell (Molleman, 2003). In study III, cell attached recordings were done from NAc neurons in coronal brain sections, by establishing a giga-seal without patching (opening) the cell. This provides a way to record the spontaneous firing activity of neurons in brain slices without rupturing the cell membrane. The technique has an advantage when studying GPCR-mediated signaling that is dependent on the presence of intracellular metabolites, such as guanosine triphosphate (GTP), and therefore is sensitive to washout-effects.

In the last decade, new techniques have evolved enabling circuit specific functional investigations, such as Chemogenetics and Optogenetics. Optogenetics is based on the discovery of algae channelrhodopsins (Chr2), which are light-gated cationchannels. These can be incorporated into neurons by utilizing the Cre-loxP system as previously described. After stable expression of $\mathrm{Chr} 2$ is achieved, laser-light can be used for activating Chr2-conduction of positive ions into the neuron. This allows for scientists, via guided control of the laser, to introduce depolarization of specific neurons with high temporal precision (Fenno, Yizhar and Deisseroth, 2011). Optogenetics have been utilized in many areas of neuroscience to study optically 
evoked release of classical amino acid neurotransmitters, such as GABA and Glutamate, using ex vivo voltage clamp recordings in slices. Yet, studies employing optic stimulation for release of neuropeptides are still rare. The reason for this is propably that neuropeptides, which are stored in large dense core vesicles, require a different pattern of optic stimulation than that used for release of fast neurotransmitters. The release of neuropeptides is overall less efficient, slower and requires longer stimulation, than that of amino acid transmitters (Arrigoni and Saper, 2014). Therefore, when recording from NAc neurons that receive input from chr2expressing POMC-projection neurons (in study III), $470 \mathrm{~nm}$ optic stimulation at 60 $\mathrm{Hz}$ was applied for minimum 1 minute.
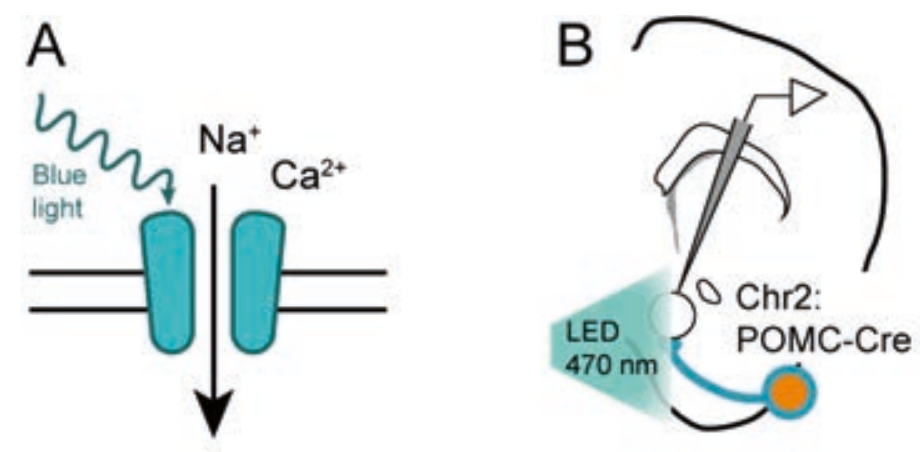

Figure 9. A schematic representation of the experimental strategy employed in study III for evoking $\alpha$-MSH release into the NAc.

A. The light-gated cation-channel, Channelrhodopsin-2, opens upon light stimulation with blue light $(470 \mathrm{~nm})$, leading to depolarization of the neuron. B. Cell-attached recordings from NAc neurons during light activation of local POMC projection-neurons. This was achieved by making slices from POMCCre-Chr2-floxed mice.

As mentioned previously, MC4Rs are excitatory receptors. Hence increases in firing activity could indicate \$-MSH release from POMC-neurons. To further complicate matters, \$-MSH is known to be co-released with glutamate (Hentges et al., 2009). Thus, in order to ensure that recorded changes in neural activity are due to MC4Rsignaling, it was pivotal to use both glutamate receptor and MC4R antagonist. Though cell-attached recordings have many advantages when monitoring differences in neural firing as a consequence GPCR-activity, they have the potential limitation of introducing erroneous signals. This is a consequence of increased somatic calcium, due to mechanical stress arising from the pipette-cell interaction, and is an effect that is more common in small neurons (Alcami et al., 2012) 


\section{RESULTS AND DISCUSSION}

In this section, the results from each study are summarized and discussed in detail. An overall discussion and perspectives on the collective research can be found in the section "concluding remarks and perspectives".

\section{STUDY I. PROSTAGLANDIN-DEPENDENT MODULATION OF DOPAMINERGIC NEUROTRANSMISSION ELICITS INFLAMMATION-INDUCED AVERSION IN MICE.}

In order to investigate how inflammation is perceived as unpleasant and causes negative affect, we developed a conditioned place aversion model towards systemic inflammation. We uncovered a specific molecular pathway underlying inflammatory aversion by employing a strategy based on cell-type specific gene deletions, pharmacology, and circuit-specific chemogenetics.

\section{Cytokine and Toll-like receptor signaling across the BBB in LPS CPA}

We found that systemic inflammation induced by a low dose of $E$. coli LPS $(10 \mu \mathrm{g} / \mathrm{kg})$ or of IL-1 $(600 \mathrm{ng})$ consistently triggered place aversion. This dose of LPS was sufficient to induce sickness symptoms such as anorexia and inactivity. In order to elucidate a potential role for BBB endothelial cells in cytokine or TLR immune-to-brain signaling, we used the floxed-Myd88 mouse in combination with the endothelial-specific Slco1c-CreERT2 mouse to achieve selective gene deletion. These animals do not develop place aversion to neither LPS nor IL-1 $\beta$. Furthermore, we uncovered that TLR4-Slco1c-CreERT2 mice also do not develop aversion to LPS, demonstrating a direct mechanism through which LPS can bind to TLRreceptors on the BBB. Endothelial recombination was validated using the Slco1cCreERT2 mice paired with a floxed tdTomato-reporter line in combination with fluorescent immunohistochemistry targeting CD31 (an endothelial specific cell adhesion molecule). To ascertain that the Myd88-signaling pathways are not involved in mediating aversion via innate immune cells, we deleted Myd88 specifically from myeloid cells using LysMCre mice. The myloid cells are precursor cells for the non-lymphoid immune cells, which include monocytes, macrophages and granulocytes (Kindt, Goldsby and Osborne, 2007). Mice with Myd88 deletion in myeloid cells displayed normal aversion to inflammation.

Since IL-1 $\beta$ aversion is Myd88-dependent, we tested different interventions targeting IL-1 $\beta$-signaling (IL-1R antagonist and global knockout, Caspase-1 knockout) to 
impact the LPS CPA phenotype, but we found these to be inadequate. We tested if $\mathrm{TNF} \alpha$ signaling in itself influences LPS CPA, but global TNF $\alpha \mathrm{R} 1 / 2 \mathrm{KO}$ did not affect aversion. The likely reason for the lack of phenotype, when targeting IL-1 $\beta$, is the previously reported compensatory mechanism by TNF $\alpha$ (Bluthé et al., 2000). In accordance with this notion, LPS CPA was significantly attenuated when testing a combined TNF $\alpha$ R1/ IL-1R knockout mouse.

\section{PGE2 signaling in the dorsal striatum via D1R neurons induce CPA}

To investigate if prostanoid synthesis is important for LPS induced aversion, we used different strategies targeting COX1 and COX2 (i.e. systemic administration of common and specific inhibitors - Indomethacin, SC560 and parecoxib respectively; i.c.v. administration of COX1 inhibitor SC560; global KO of COX 1 and COX2). These results coherently revealed that COX1, and not COX2, is the major isoform involved in prostanoid synthesis signaling inflammatory aversion. Furthermore, we found that the mPGES1 KO mice have significantly attenuated LPS CPA, which demonstrates an important role for PGE2 production in systemic inflammation induced aversion. In order to explore the role of PGE2 receptors, we tested EP1R, EP2R, EP3R and EP4R-Nestin KO mice in the LPS CPA paradigm. Interestingly, both EP1 and EP2 receptor deletion significantly attenuate inflammation-induced aversion. The EP1Rs have been demonstrated to regulate striatonigral circuitry in relation to stress (Tanaka et al., 2009; 2012). Hence it was not surprising to find that local injections of PGE2 into the $\mathrm{CPu}$ elicits CPA, while injections into the NAc failed to change the initial choice of chamber. This effect was due to EP1Rs specifically on striatal D1R expressing neurons, as illustrated using rescue-strategies targeting $\mathrm{D} 1 \mathrm{Rs}$ and the $\mathrm{CPu}$ (floxed-stop-cassette $\mathrm{KO}$ mice and AAV5-Cre respectively). Validation of recombination was done using Cre-dependent fluorescent reporter proteins, i.e. the floxed tdTomato mouse line or AAV5-DIOeGFP, in combination with immunohistochemistry targeting either enkephalinpositive D2R MSNs (to investigate specificity towards D1R over D2R expressing neurons) or eGFP for reinforcing the viral signal.

\section{Inflammation leads to decreased dopamine mediating aversion}

In coherence with the above-mentioned findings, demonstrating the importance of PGE2-signaling in the dorsal striatum during inflammation-induced CPA, PGE2metabolite ELISAs confirmed significant induction of PGE2 in the $\mathrm{CPu}$, but not in the NAc. This induction was dependent on COX1-activity as it was absent in COX1KO mice. In a following series of experiments, we found that interventions targeting dopaminergic circuitry were successful against LPS CPA. When diminishing GABAergic input to dopamine neurons, by targeting the GABAA-receptor subunit 
Gabrg2 specifically on dopaminergic neurons via the DATCreERT2 mouse, place aversion to inflammation was abolished. When using hM3Dq DREADDs to activate midbrain dopamine neurons prior to and during conditioning with LPS, mice did not develop aversion. Recombination was validated for these two strategies by employing Cre-dependent fluorescent reporters (tdTomato and mCherry) in combination with fluorescent immunohistochemistry targeting tyrosine hydroxylase positive neurons in the midbrain (VTA and SN). In line with these findings, blocking dopaminergic-signaling with a D1R-antagonist, SCH23390, restored inflammatory place aversion in EP1R KO mice. Finally, we demonstrated that inflammationinduced aversion is not an indirect consequence of anorexia or pain, but instead constitutes an independent inflammatory symptom triggered by a unique molecular mechanism. We did this by targeting molecules that were critical for eliciting positive results in the CPA-paradigm: COX1 inhibition, Myd88-Slco1c-CreERT2, EP1R KO, EP1R-D1RCreERT2 rescue and Gabrg2-DATCreERT2. None of these interventions affected inflammation induced anorexia or pain CPA.

\section{STUDY I. DISCUSSION}

Collectively, the findings from study I demonstrate a pathway underlying negative motivational state induced by inflammation, in which peripheral cytokines and PAMPs signal across the BBB leading to PGE2-mediated modulation of the dopaminergic motivational circuitry.

\section{Inflammation induced conditioned place aversion - a new way of monitoring inflammation induced negative affect?}

All results from this study are novel, as we are the first to publish findings from the LPS CPA paradigm. Previous studies exist with conditioning-protocols towards inflammatory stimuli; these are primarily CTA paradigms, but there also exists one IL-1 $\beta$ odor-based conditioning paradigm published by Morméde and colleagues (2003). Unfortunately, the paradigm used in that study is unlikely to be comparable with ours, as the authors did not utilize a classical place-conditioning protocol. Furthermore, inflammation induced CTA has been demonstrated to be independent of PGE2-synthesis (Nilsson et al., 2017a), hence these models represent different inflammatory behaviors with specific neurobiological foundations.

Monitoring negative affect related to systemic inflammation in preclinical models is not straightforward. Cytokine-induced sickness behaviors represent a potential bias in classical behavioral paradigms, such as the forced swim test and sucrose preference test. This is because some sickness behaviors overlap with preclinical depression-like behaviors. In particular, decreases in locomotor activity and food 
intake are major symptoms of the sickness syndrome, which interfere with preclinical readouts for depressive state. For instance, both the forced swim and tail suspension tests operate with immobility as an equivalent to helplessness and despair behavior, while decreased sucrose intake in the sucrose preference test is interpreted as depression-associated anhedonia. In this context, the LPS CPA paradigm offers an advantage, as there is no inflammatory stimulus present on the test-day. The mice are making their choice in respect to the previously formed association between the inflammatory stimulus and the cues. In this way, the actual symptoms arising from the systemic inflammation cannot influence the behavioral readout of this model.

As mentioned under the methodological considerations, this separation of the affective experience during the conditioning from the learned response measured during the test is unfortunately also one of the limitations of the paradigm. A major concern, for the interpretation of conditioning results, is the lack of separation between effects related to acquisition and expression. In the present study, we were able to approach the issue of integrity of transgenic mice in acquisition by utilizing the formalin paw pain paradigm. In other words, we proved that the mice were not unable to learn the conditioning scheme. Some of the most important findings in this study demonstrate the difference between the pathway signaling inflammationinduced aversion and other inflammatory pathways responsible for anorexia, pain and fever. We found that the interventions blocking aversion had no effect on loss of appetite or pain. Furthermore, it has been demonstrated in the literature that fever is mediated by COX2 and EP3Rs, rather than COX1 and EP1Rs. This makes the systemic inflammation induced aversion particularly interesting, as it constitutes a previously undefined (though for most people well-known) symptom of the sickness syndrome. Consequently, the LPS CPA paradigm may offer a whole new avenue of research relevant for understanding how the immune system directly influences motivational state.

In the Merriam-Webster dictionary affect is defined as a conscious subjective aspect of an emotion. In the current study, we assumed that inflammatory CPA functions as readout for changed motivational state due to negative affect (i.e. malaise and decreased mood). This notion is equivalent to findings from human CPP studies showing that chamber choice reflects subjective feelings associated with the US. In the case of the LPS CPA using transgenic mice, this conclusion may be considered premature for the following reasons: 1) We did not utilize any other behavioral paradigms to monitor affective state because it is not possible to separate sickness behavior and mood effects during acute inflammation - as discussed above. We could have utilized previous inflammation induced depression models, but they offer very poor translatability as they are based on non-acute administrations of very high 
doses of inflammatory stimuli. 2) The overlay between motivational circuitry and affective neurocircuitry remains to be clarified. As explained in the introduction on "the role of dopamine", Berridge, Robinson and Salamone demonstrated how dopamine encodes incentive and not affect. Despite the clear neurobiological segregation between affect and motivation, they are undeniably related. We are by nature motivated to seek pleasant stimuli and changes in dopamine signaling is associated with changed affective state.

\section{Immune-to-brain signaling across the BBB - the missing link to CPu PGE2- synthesis and the missing cell}

The finding that Myd88 deletion from endothelial cells prevents aversion to both LPS and IL- $1 \beta$ is particularly interesting. It demonstrates the presence of a pathway across the BBB, not only restricted to PAMPs (via TLR4s), but also utilized by cytokines involved in various inflammatory conditions. It is important to note that though we validate brain-endothelial recombination using the tdTomato reporter, the Slco1c-CreERT2 mouse has been found to also induce some recombination in other organs. We base our results on the cumulative evidence, demonstrating the importance of BBB endothelial cells, arising from other strategies (Ching et al., 2007; Wohleb et al., 2014).

As it has been demonstrated that peripheral cytokines induce synthesis of PGE2 in the endothelium during fever, it is not unlikely that a similar mechanism is involved in inflammatory aversion. It would have been relevant to provide data supporting the molecular link between peripheral inflammation and PGE2 synthesis in the CPu. Though we demonstrate that these features collectively are central for the same behavioral readout (the LPS CPA), we do not show any molecular data supporting the causal link between the pathways. Experiments could have been pursued targeting COX1 or mPGES1-expression or detecting PGE2-levels in the Myd88- or TLR4-Slco1c-CreERT2 mice. However, COX1 induction remains controversial and it is unknown if mPGES1 expression changes during low doses of LPS.

An interesting feature of the inflammation-induced aversion is COX1 and mPGES1 coupling. This is considered somewhat controversial in the field of immune-to-brain signaling. Fortunately, studies are emerging with evidence for such a link during various inflammatory conditions. A question still remains though: Which cell-type is responsible for PGE2 synthesis leading to aversion? In the article, we hypothesized that it would be endothelial cells expressing COX1 and mPGES1, though COX1 is primarily expressed in microglial cells. We are currently working on a follow-up study that has demonstrated that COX1 in endothelial cells is not responsible for LPS 
CPA. Instead, we found that prostanoid synthesis in microglia is mediating the aversive signaling and $\mathrm{MCP} 1$ released from the $\mathrm{BBB}$ endothelium is involved. As a consequence, microglial mPGES1 should be the isomerase responsible for PGE2 synthesis, but this remains to be confirmed. Primary culture studies have demonstrated the presence of microglial mPGES1 PGE2 synthesis (Ikeda-Matsuo et al., 2005), while results arising from brain immunohistochemistry have not provided conclusive evidence for this (Eskilsson et al., 2014; Ikeda-Matsuo et al., 2005).

\section{PGE2 signaling to motivational neurocircuitry - Time for a revision of the role of nigrostriatal circuits in motivation?}

Another central finding is that PGE2 induction in the $\mathrm{CPu}$ leads to aversion via EP1Rs on D1R-expressing neurons. This result is interesting as increased activity of striatal direct pathway neurons commonly is considered to signal reward. The direct pathway neurons mediate some of these effects by targeting GABAergic interneurons in the midbrain, which disinhibit dopamine neurons (Bocklish et al., 2013). Tanaka et al (2009) demonstrated that EP1Rs located on GABAergic neurons target the dopamine neurons directly, thereby leading to a decrease in dopamine. Our results are consistent with these findings. The specific differences in direct-pathway circuitry of the striatum remains to be further explored. In this context our findings are somewhat controversial, as it is generally regarded that D2R indirect pathway neurons are the key players in signaling aversion. The finding that EP1Rs on D1R neurons are responsible for reducing dopamine, could demonstrate a collective strategy through which Gq-coupled EP1Rs on both D1R and D2R MSNs mediate similar effects in the presence of PGE2. This hypothesis remains however to be explored. Furthermore, targeting EP2Rs demonstrated similar effects as EP1Rs. These receptors could be interesting to explore further in inflammation induced aversion and affective state, as they are expressed on affective neurocircuitry and display a somewhat different expression patter than EP1Rs.

The finding that PGE2 drives aversion when injected in the $\mathrm{CPu}$, but not the NAc, demonstrates the specificity of the PGE2-signaling. Yet, this is somewhat surprising, since the $\mathrm{CPu}$ primarily is involved in habituated behaviors, while the NAc is central for acquisition. Transfer of motivated associations is believed to occur through an acceding spiral from the mesolimbic system towards striatonigral circuitry (Keifling and Janak, 2015). A possible hypothesis could be that the behaviors related to avoiding diseases already are integrated, as experiencing infections occur early in life. The extent to which this is the case in our mice is difficult to estimate, as they are kept in relatively pathogen-free facilities. An alternative hypothesis could be that negative events are habituated faster than positive. This seems plausible, as it is known that negative associations are more difficult to extinct than positive ones. 
Most importantly, several studies have demonstrated the involvement of the dorsal striatum in both aversion and reward, as mentioned in the introduction. Maybe it is time to revise the concept of a clear segregation between NAc and $\mathrm{CPu}$ in motivated behavior?

The chemogenetic activation of dopamine neurons preventing LPS CPA should have been further scrutinized to ensure that the result is not due to a counterconditioning effect induced by dopamine. This experiment could easily have been done with hM3Dq-DATCreERT2 animals using the same activation-protocol, but in the absence of LPS. Therefore the chemogenetic activation results should be considered supportive. Optimally, a direct measure of inflammation-induced changes in dopamine should have been provided. We conducted in vivo microdialysis post LPS administration, but the HPLC-technique was not sensitive enough to detect decreases in dopamine. However, we found the hypothesized LPS decrease in dopamine in wildtype mice using $\left[{ }^{11} \mathrm{C}\right]$ Raclopride PET-scans in study III.

In conclusion, the results from our study suggest several relevant targets for treating the aversive component of systemic inflammation, including COX1, mPGES1, EP1 and EP2 receptors, which may alleviate negative affect during acute inflammation, and perhaps also in chronic inflammatory diseases.

\section{STUDY II. INTERFERON- $\gamma$ MEDIATED SIGNALING IN THE BRAIN ENDOTHELIUM IS CRITICAL FOR INFLAMMATION-INDUCED AVERSION}

In study I, we uncovered mechanisms underpinning the aversive component of inflammation, which include striatal PGE2 synthesis and modulation of dopaminergic circuitry, but the messengers mediating the signal between the peripheral inflammation and the brain have not been sufficiently characterized. In study II, we investigate the role of peripheral IFN- $\gamma$ in LPS induced conditioned place aversion by employing a strategy based on global and cell-type specific gene deletions, combined with measures of gene-expression.

We found that an acute injection of LPS, corresponding to the CPA conditioning dose, caused significant induction of IFN- $\gamma$ expression in the blood of wild type mice. Interestingly, global deletion of IFN- $\gamma$ abolished LPS induced aversion. In line with this finding, abolishing the IFN- $\gamma \mathrm{R}$ led to a similar effect. These results indicate the relevance of IFN- $\gamma$ peripheral signaling in systemic inflammation. Furthermore, the mRNA of the IFN- $\gamma$-induced chemokine Cxcl10 was significantly elevated in the dorsal striatum after an acute LPS injection. This induction was dependent on IFN- $\gamma$ signaling, as it was absent in global IFN- $\gamma \mathrm{R}$ KO mice. Similar to the result from mice 
lacking IFN- $\gamma$, LPS induced aversion was absent in mice lacking Cxcl10. In line with this, global deletion of the Cxcl10-receptor Cxcr3 prevented inflammation-induced aversion. In order to assess if IFN- $\gamma$ signaling leading to aversion is dependent on a BBB-endothelial mechanism, as is the case for the pro-inflammatory cytokine IL-1 $\beta$ and the type I interferons, we deleted IFN- $\gamma$ Rs specifically from endothelial cells using the floxed IFN- $\gamma$ R and Slco1c-CreERT2 mouse lines. Recombination in BBB endothelial cells of the striatum was validated using the floxed tdTomato reporter line in combination with immunohistochemistry targeting the endothelium specific protein lipocalin-2. Furthermore, endothelial specific IFN- $\gamma$ R knockout was assessed in whole brain and peripheral organs (liver, kidney and spleen) with qPCR. Unfortunately, the Slco1c-CreERT2 strategy is not completely specific to BBB endothelial cells, as knockout also occurs in the spleen. In the same manner as global IFN- $\gamma$ R knockout, endothelial specific abolishment of IFN- $\gamma$ Rs prevents LPS CPA. In addition, we found that striatal Cxcl10-induction is dependent on endothelial activation by Myd88, as the response was absent in mice lacking Myd88 from BBB endothelial cells.

\section{STUDY II. DISCUSSION}

Collectively, the findings from study II suggest a mechanism by which peripheral IFN- $\gamma$ binds to IFN- $\gamma$ receptors and induces endothelial activation, which in turn upregulates Cxcl10 that binds to Cxcr3-receptors in the striatum and causes inflammation induced aversion.

\section{Cytokines signal across the BBB endothelium via Myd88}

\section{- All paths lead to Rome}

IFN- $\gamma$ signaling produces the strongest effect on LPS CPA in comparison with other cytokines, as the aversive response to inflammation is completely absent when intervening with IFN- $\gamma$ Rs. On the contrary to the other pro-inflammatory cytokines, IL-1 $\beta$ and $\mathrm{TNF} \alpha$, there is no compensatory mechanisms for its absence. Irrespectively, many aspects of the IFN- $\gamma$ signaling pathway mirror the mechanism uncovered in study I, but it remains to be explored how these two relate, and what their roles are in PGE2 synthesis. An important feature of the peripheral immune-tobrain LPS aversion pathways is the involvement of Myd88-dependent signaling in BBB endothelial cells. It has previously been demonstrated that IFN- $\gamma$ Rs recruit Myd88. In the present study, we found that upregulation of the downstream signaling molecule, Cxc110, is Myd88-dependent. This may reflect a general biological principle where activation of toll-like receptors and cytokine receptors converge in brain endothelial cells. 
The development of parallel systems signaling inflammation induced negative affective state, by utilizing the same BBB endothelial mechanism, clearly demonstrates the evolutionary importance of this behavioral response. The question remains if these systems are interdependent or independent. To investigate this in more detail a parallel experiment to the IL- $1 \beta$ CPA in study I could have been conducted: IFN- $\gamma$ CPA with Myd88-Slco1c-CreERT2 mice. In general, the question of interdependence of signaling pathways constitutes a conundrum in behavioral neuroscience, since similar experimental conditions can be blocked by a broad array of interventions. Are these targets all part of one giant interdependent network? Or are we affecting difference aspects leading to prevention of the same behavior?

As discussed in the previous section on study $\mathbf{I}$ and in the methodological considerations the Slco1c-CreERT2 strategy is not specific to BBB endothelial cells. This is a limitation of the strategy, which implies that the relevance of our results relies on supportive evidence from the literature (Ching et al., 2007; Wohleb et al., 2014). Though it should be noted that it is unlikely that the spleen endothelial cells are directly involved in immune signaling to the brain.

\section{Interferons and Cxcl10 in negative affect - A new microglia hypothesis}

An advantage of study II is the use of expression analysis to complement the conclusions reached from the global knockout mice in LPS CPA. This helps us to investigate the causality between IFN- $\gamma$-signaling and downstream target, Cxcl10, in a brain area-specific manner. However, we do not clarify which brain-cells the Cxcl10 target. The changes observed by Blank et al (2016) in depressive phenotype were concluded to be due to Cxcr3Rs directly on neurons. Nevertheless, as the interventions targeting Cxcr3Rs were bath-applied to brain sections, the authors cannot exclude involvement of microglia, as both neurons and microglia express Cxcr3Rs. The authors excluded microglia as key-players, since they did not see upregulation of the microglial activity marker major histocompatibility complex II (MCHII); whether this is a sufficient exclusion criterion is debatable.

There are many similarities between the Interferon type I pathway and the one we discovered for IFN- $\gamma$, implying that the IFN- $\gamma$ mechanism might be generalizable to depressive behaviors. IFN- $\gamma$ has previously been implied in depressive phenotype via induction of IDO, which is highly expressed in microglia. It could be interesting to explore if IDO is a downstream target of IFN- $\gamma \mathrm{R}$ induced Cxcl10. A hypothesis could be, that IFN- $\gamma$ through BBB endothelial activation leads to microglia induction of IDO via Cxcr3R-signaling. Yet, the link between Cxcr3Rs and microglial IDO remains to be explored. 


\section{STUDY III. MOTIVATIONAL VALENCE IS DETERMINED BY STRIATAL MC4 RECEPTORS}

Aversive signaling causes extensive suffering during chronic diseases including inflammatory conditions, cancer and depression. Here we investigated the role of MC4Rs in aversive processing using genetically modified mice in conditioned place aversion to various stimuli. We further explored the POMC-projection neurons responsible for activating MC4Rs using circuit-activating chemogenetics in behavior and in combination with PET-scans.

In normal mice, robust aversions were induced by systemic inflammation, nausea, pain and kappa opioid receptor-induced dysphoria. In sharp contrast, mice lacking MC4Rs displayed preference to all stimuli except pain, which they were indifferent towards. Furthermore, inflammation induced aversion was flipped to preference in normal wildtype mice by intranasal administration of a MC4R antagonist prior to conditioning. This result demonstrates that the preferences to sickening stimuli in the absence of MC4R-signaling is not due to adaptive or secondary mechanisms. Interestingly, we found that MC4R KO mice still develop normal sickness responses in the same manner as wildtype mice to both systemic inflammation (anorexia and $\mathrm{HPA}$-axis activation) and $\mathrm{LiCl}$ (nausea induced hypothermia). Finally, the absence of MC4R signaling did not interfere with reward encoding, as knockout mice respond normally to place conditioning towards positive stimuli (cocaine and palatable food). Administration of a D1R antagonist (SCH23390 i.p.) prior to conditioning restored inflammation, dysphoria and pain aversion in mice lacking MC4Rs. This suggests that the unusual flip from aversion to reward is dopamine-dependent. Furthermore, inhibiting midbrain dopamine neurons using inhibitory DREADDs (AAV8-hM4Di) in MC4R KO mice elicits aversion in the same manner as in normal mice. This finding exemplifies a type of aversion that MC4R KO mice are able to perceive, which implies that dopamine activation is necessary for these mice to flip aversion to reward. Expression was validated using the fluorescent reporter protein mCherry, which is part of the viral construct, combined with immunohistochemistry with an RFP-antibody for signal amplification. Moreover, PET-scans of dopamine receptor ligand $\left[{ }^{11} \mathrm{C}\right]$ raclopride displacement illustrated that dopamine is decreased in normal mice in response to inflammation. However, in the MC4R knockout mice inflammation is associated with a change from decreased to increased activity of the dopamine system. The dopamine increase occurs specifically in the NAc shell. This finding provides a biological foundation for the observed flip the animals display in the conditioned place aversion paradigm. 
Based on our current findings and previous studies on striatal MC4Rs (Lim et al., 2012), we investigated the expression of MC4Rs on D1R neurons in $\mathrm{CPu}$ and NAc. This was achieved using transgenic mice expressing eGFP under control of the MC4R promoter and tdTomato under control of the D1R promoter. These results confirmed co-expression of MC4Rs with D1Rs on a subset of neurons in both parts of the striatum. Responses to aversive stimuli (inflammation, dysphoria and pain) were normalized when MC4Rs were re-expressed on dopamine D1 receptorexpressing cells. This was achieved by employing D1RCreERT2 to excise the floxed-stop-cassette, whereas re-expressing MC4Rs on dopamine neurons using DATCreERT2 mice did not restore inflammation-induced aversion. More specifically, we found that re-introducing MC4Rs via AAV5-Cre in the striatum reestablished LPS CPA. Recombination was validated using AAV5-DIO-eGFP injected together with the AAV5-Cre, in combination with immunohistochemistry against eGFP for increasing the signal.

Finally, we investigated the function of neurons signaling to the striatal MC4R population responsible for the flip from aversion to preference. To detect the motivational valence encoded by ARC POMC-neurons we used hM3Dq-activation in a place-conditioning task. In the first round of experiments the AAV8-DIO-eGFP was injected in the ARC of POMCCre mice for direct targeting of this population. Subsequently we targeted the specific POMC-projection to the NAc shell by retrograde transduction in POMCCre mice. Both direct ARC POMC-neuron activation and ARC-NAc POMC-neuron activation induced significant conditioned place aversion. Expression and recombination were validated employing vectorexpression of mCherry combined with immunohistochemistry against RFP. These results demonstrated specific and adequate viral expression in ARC POMC-neurons, and retrograde transduction specifically from NAc-shell to ARC, without transduction of POMC-neurons in the NTS. Functional validation of the ARC-NAc POMC circuitry to MC4R neurons was carried out using optogenetics combined with cell-attached recordings from NAc sections of Chr2-POMCCre mice. We found that POMC projection activation increases neural firing in a glutamate and MC4R dependent manner in a subset of NAc neurons. Likewise, PET-scan experiments employing DREAMM (DREADD-assisted-metabolic-mapping) confirmed the striatal activation during ARC-to-NAc POMC-neuron activation. 


\section{STUDY III. DISCUSSION}

Collectively our findings from study III demonstrate that melanocortin signaling through striatal MC4Rs is critical for assigning negative motivational valence to harmful stimuli by inhibiting dopamine signaling.

Intriguingly, the aversions to most stimuli were flipped into preferences in the mutant mice. In response to LPS, we consistently saw preferences in MC4R KO mice. Pooled analysis of all results from naive MC4R KO mice given LPS produces a preference score that is highly significantly different from zero $(\mathrm{p}=0.0005$; column statistics, one sample t-test). This strongly suggests that the knockout mice develop a real preference and not only a lack of aversion. However, the absence of an aversive phenotype is stronger and more generalizable than the preferencephenotype, and the increase in dopamine signaling upon LPS was less extensive than the drop seen in wildtype mice.

It is conceptually interesting to discuss the preference phenotype occurring in some of the experiments. For instance, the question arises on the separation of motivation and affect: Do the MC4R KO mice develop preferences to sickening stimuli because they perceive them as pleasant? Or is this just a strange wanting of something that actually was associated with negative affect? There are two minor findings, which indicate absence of negative affect associated with aversive stimuli in MC4R knockout mice: During temperature measurements, it is common that mice display a slight temperature elevation upon handling due to stress, this reaction is significantly decreased in the MC4R knockout mice. In line with this they also display a slight reduction in the late corticosterone response to LPS. These findings would indicate that the knockout mice are less vulnerable to stress, which could be related to decreased negative affect. As considered in study $\mathbf{I}$, when using knockout mice in CPA, we cannot separate affective/valence state during conditioning from the consequential learning observed on the test-day. However, we did explore acquisition of behaviors in the place preference paradigm using a MC4R antagonist administered prior to conditioning, which showed a similar effect as the knockout strategy. To further confirm this finding, it could have been relevant to test the MC4R antagonist against the already learned behavior (i.e. on expression).

In this context, it is relevant to note that the MC4R knockout mice have been suggested to exhibit procedural memory deficits, but these findings are highly debatable (as mentioned in the introduction). On the contrary, we demonstrated that MC4R KOs are perfectly capable of learning both place conditioning and procedural operant responding towards rewards. 
Moreover, MC4Rs have been suggested to be involved in inflammation-induced appetite-loss, but we found an intact LPS-induced anorexia in the MC4R KO mice. The intact anorexia in combination with the blocked aversion correspond to our findings in study I suggesting that the affective and the anorexic component of systemic inflammation are mediated by different circuits. Additionally, the use of chemogenetics and optogenetics for activation of ARC AgRP and POMC neurons has demonstrated completely different temporal effects on food-intake. AgRPactivation leads to immediate consummatory behavior, which is independent on MC4Rs, whereas POMC-neurons produce late onset responses (Aponte et al., 2011; Atasoy et al., 2012; Zhan et al., 2013). In our study, we see fast effects of POMCactivation on motivated behavior, indicating that this circuit-function is distinct from that signaling satiety. Conditioning towards chemogenetic activation lasts 1 hour in our study, whereas Aponte and colleagues (2011) used 24 hours ongoing photostimulation to evoke POMC-induced decreases in food-intake. In this manner potential POMC-satiety effects would occur when the animals are back in their homecages, preventing the association between conditioning cues and this effect. Therefore, it is unlikely that satiety effects would affect the results from our conditioning paradigm.

Furthermore, the results arising from MC4R-rescue strategies are particularly important as they strengthen the conclusion that the observed behavioral phenotype of the knockout mice is specifically related to absence of the receptor and not due to secondary or adaptive biological alterations. This strategy provided data illustrating the specificity of the MC4Rs on D1R neurons in the striatum for encoding negative valence.

Our findings demonstrate that POMC neurons projecting to the ventral striatum induce aversion by activation of MC4Rs. In this way, the natural circuitry promoting MC4R signaling encodes negative valence. But how about the observed flip in the case of absence or antagonism of MC4R function - is it likely to occur in nature? Since AgRP is the natural antagonist of MC4Rs, a potential biological correlate to MC4R knockout could be the AgRP neurons projecting to the NAc. In contrast to this idea, Betley and colleagues found that direct stimulation of ARC AgRP neurons leads to place avoidance (Betley et al., 2015). This is conceptually logic, as AgRPneurons signal hunger and because starvation is a physiologically aversive state. However, different AgRP neurons may exhibit specific effects. It could be that some neurons play a role in food-intake and others signal positive affect either during normal conditions or as a compensatory mechanism during stress. Substantially more work needs to be done to clarify the biological function of the MC4R-circuitry in 
affective state. It could be relevant in this context to explore the ARC AgRP to NAc shell projection.

In contrast to the excitatory POMC-projections to the NAc, most other excitatory projections to the ventral striatum signal reward. Activation of glutamatergic input from the ventral hippocampus, the basolateral amygdala and the prefrontal cortex all induce place preference (Stuber et al., 2011; Britt et al., 2012). Similar to the POMC projection, glutamatergic input from the ventral tegmental area and the periventricular thalamic nucleus both induce aversion, but they do so by targeting parvalbumin positive GABAergic interneurons and D2R expressing medium spiny neurons (Delfs et al., 2000; Zhu et al., 2016; Qi et al., 2016). In contrast, our findings indicate that POMC neurons signal aversion by activation of a sub-population of D1R neurons. In line with this, stress-induced anhedonia is mediated by MC4Rdependent synaptic plasticity in D1R MSNs of the ventral striatum. In study I we also found an aversive mechanism through excitatory (EP1R Gq-mediated) D1R MSN stimulation, which is assumed to directly target dopaminergic neurons leading to decreased dopamine transmission and hence aversion. MC4Rs could directly influence dopamine tone by utilizing a similar mechanism. The only known neurocircuitry capable of mediating bi-directional valence is the midbrain rewardprediction error dopamine neurons. In this context, it is not surprising that the motivational flip was associated with a flip in dopaminergic signaling. Nevertheless, it remains a question if the MC4R expressing D1R neurons mediate this flip by directly targeting dopaminergic neurons, as suggested above. Such a neurocircuit mechanism could during MC4R activation mediate dopamine inhibition causing aversion, while MC4R antagonism could trigger disinhibition leading to increased dopamine and preference. In line with this idea, the concept of specific subsets of midbrain dopamine neurons responding directly to sickness and stress or oppositely relief and recovery seems relevant. Lammel and colleagues (2012) from the Malenka laboratory elegantly introduced the idea that specific subsets of dopamine neurons are responsible for different behavioral output dependent on the origin of the input they receive. It would have been highly interesting to activate or inhibit the MC4RD1R circuitry using optogenetics or chemogenetics for uncovering their projection targets. This would allow us to explore the underlying biology of the potential motivational flip and characterize this unique population of direct-pathway neurons in the striatum. For this purpose one could utilize the MC4RCre mouse for anterograde and subsequently retrograde activation of specific MC4R neuropopulations dependent on their projection targets. Unfortunately, we were unable to acquire the MC4RCre mouse for necessary for these experiments. 
The hypothetical description of D1R-neurons directly synapsing on dopamine neurons leading to the motivational flip could be interesting, but it does not explain the biological correlates we observed related to the behaviorally robust absence of aversion. The $\left[{ }^{11} \mathrm{C}\right]$ raclopride displacement results revealed an extensive decrease in dopamine throughout the brain of normal mice in response to inflammation, in comparison to the modest increase of dopamine in the NAc shell of MC4R KO mice. The absence of dopamine-decrease throughout the brain during MC4R knockout is substantial, and therefore cannot be due to the same population of neurons responsible for the minor dopamine increase in the MC4R KO mice. There must exist another circuit responsible for this phenomenon. The answer may still be found in a mechanism involving direct targeting of dopamine neurons by the striatal MC4R-D1R neurons. However, the difference between the broad absence of 'decrease in dopamine' throughout the brain and the small increase in NAc dopamine could lie in the function of subpopulations of dopamine neurons. The different functionality of dopamine neurons could arise from specific input from other brain-areas responsible for controlling their excitability.

In this study, we show that MC4Rs localized on striatal D1R MSNs control behavioral responses to a variety of aversive stimuli and thereby play a generalized role in aversive processing. Our findings indicate that MC4Rs assign negative valence to salient stimuli, effectively acting as gatekeepers, whose role is to hinder aversive signals from inducing reward. Such a mechanism has obvious adaptive value for survival, as it is likely to encode a fast and substantial response to any kind of aversive stimuli or situation. However, in chronically ill patients this pathway may constitute an unwanted reason for suffering. The MC4R KO mice exhibit intact sickness symptoms, which is an advantage as they have a normal biological response to sickening stimuli. This is an important feature for the applicability of targeting this system for treatment of negative affect during disease, while sickness symptoms often are an important part of optimizing the organism to combat or adapt to disease. In our study, we found that intranasal administration of a MC4R antagonist had strikingly similar effects to MC4R knockout on aversive phenotypes. Recently, intranasal peptide treatments have been developed as a way to circumvent the limited ability of peptides to traverse the BBB. Our findings indicate that MC4R antagonism has the potential to be utilized as a generalized treatment against aversive states. 


\section{Study IV. Muscarinic M4 receptors on cholinergic and D1R-expressing neurons have opposing functionality for positive reinforcement and influence impulsivity}

The neurotransmitter acetylcholine has been implied in reward learning and drug addiction. However, the role of the various cholinergic receptor subtypes in such processes remains elusive. Here we study the function of muscarinic M4Rs on dopamine D1R expressing neurons and cholinergic neurons, using transgenic mice in various reward-enforced behaviors and in a "waiting"-impulsivity test.

In study IV, we used the floxed M4R mouse line combined with either D1RCre or ChATCre mouse lines, in order to achieve cell specific deletions from these two types of neurons. Neither M4R-D1RCre nor M4R-ChATCre mice differed from wildtype littermates during baseline locomotion. But when comparing the acute effect of cocaine between wildtype and knockout animals, the M4R-D1RCre mice displayed an increased locomotor response. Both M4R-D1RCre mice and wildtype mice developed locomotor sensitization after repeated injections of cocaine, but the sensitization of M4R-D1RCre mice was significantly higher than that of the control mice. In this way, the M4-D1R KO mice display a disinhibited phenotype to psychostimulants as previously shown in the literature (Jeon et al., 2010). In contrast, no differences in acute or sensitization effects to cocaine were observed in the M4RChATCre mice. In line with these results, we found that selective deletion of M4Rs from D1R expressing neurons led to increased cocaine CPP acquisition and drug primed reinstatement. Mice missing M4Rs from cholinergic neurons had a significantly attenuated cocaine place preference compared to controls. The same tendencies for M4R-D1RCre and M4R-ChATCre mice as in the cocaine CPP were observed for palatable food CPP, but no significant differences were present between the groups. To evaluate the role of the two M4R populations for the expression of immediate early genes involved in neural activity, we investigated expression of cFos, FosB, Egr1, Egr2 and Arc after four consecutive days with cocaine injections. Accordingly, cocaine induced significant increases in cFos and FosB in the forebrain of M4R-D1RCre mice compared to control mice, while no significant differences were observed after cocaine between the M4R-ChatCre and control mice.

We then went on to explore the behavior of M4R-D1RCre and M4R-ChatCre mice in operant conditioning. No differences in operant responding were observed between mice missing M4Rs from D1R expressing neurons and control animals, while mice lacking M4-autoreceptors were unable to learn the operant runway task to both cocaine and palatable food. Next, we tested the M4R-D1RCre mice in the 5choice serial reaction time task (5-CSRTT) to study if they exhibited changes in impulsivity and learning behaviors. The M4-D1R KO mice learned the reward task better than their wildtype littermates, as they obtained a significantly higher amount 
of rewards under baseline conditions. Moreover, the M4R-D1R knockout mice showed an initial increase in waiting impulsivity, as they had significantly more premature responses. Yet, the M4-D1R knockout mice adapted to the task over the subsequent trials and performed comparable to normal mice. The same phenomenon was observed for the accuracy (pressing the correct window). Initially during the first test, the mice lacking M4Rs from D1R neurons had lower response accuracy than control mice, but still managed to learn the task equivalently well over the next trials. For further details on the 5-CSRTT, please see paper IV.

\section{STUDY IV. DISCUSSION}

Collectively, our results demonstrate how muscarinic acetylcholine receptors on cholinergic and D1R expressing neurons are involved in regulating reward learning and impulsivity, with opposing functionality.

It is clear from our study that the M4-receptors on dopaminoceptive D1R-neurons play an important role in reducing the reinforcing effects of both natural rewards and drugs-of-abuse. The M4R-D1RCre mice displayed an exacerbated response to rewards throughout all paradigms, except the operant runway. A possible reason for this difference could be the practical limitations of the runway paradigm. The animals decreased their runtime as fast as the controls, but there is a ceiling-effect on runtime due to the length of the runway, preventing us from seeing differences arising from decreased runtime. To explore phenotype-specific decreases in runtime, it would be relevant to adjust either the length of the runway or separate the runningtrials to different days. Increasing the duration of the runway-protocol to several days instead of one could prevent the accumulative cocaine effect on locomotion.

However, the operant runway has the advantage that it complements the conditioned place preference cocaine seeking behavior since its operandum is running to the drug-paired compartment at the end of the corridor (i.e. seeking the drug). The disadvantage with the operant runway, compared to operant self-administration, is that it is not possible to take the animals through a progressive ratio schedule in order to measure breakpoint, i.e. how much the animals are willing to work for the drug. Irrespectively, we did get some interesting information from the operant runway. The M4R-ChATCre mice did not develop any search behavior and therefore, just randomly explored the corridor as if the cocaine had not been administered. It was clear that there were no U-turn effects to the cocaine. U-turn behavior in the runway typically indicates the aversive/b-process component of cocaine. In this manner the absence of operant responding in the M4R-ChATCre mice is more likely due to lack of reward learning, rather than a shift in pharmacological sensitivity or changes in affective neurocircuitry. 
The exacerbated reward-phenotype related to M4R knockout from D1R-neurons is likely to be a consequence of increased dopaminergic transmission. This is in accordance with previous observations that the M4R-D1RCre mice exhibit elevated dopamine-release in the striatum (Jeon et al., 2010) and the occurrence of exaggerated cocaine induced locomotor sensitization in these mice. Locomotor sensitization is in general believed to be a physiological consequence of synaptic changes occurring within the dopaminergic system in response to drugs of abuse (Steketee and Kalivas, 2011).

In addition, it has previously been shown that D1R-medium spiny neurons of the NAc, projecting to GABAergic interneurons in the VTA, are involved in cocaineinduced disinhibition of VTA dopamine neurons (Bocklish et al., 2013). Hence relief of inhibition of the NAc D1R medium spiny neurons, by removal of the inhibitory M4Rs, could be a mechanism involved in the augmented dopamine and reinforcement response. The finding that markers of neuronal activity (cFos and FosB) were significantly elevated in the forebrain of these animals compared to control mice, would support this line of thinking. Changes in mesolimbic neurotransmission could also explain the impulsive phenotype observed in the 5CSRTT paradigm.

\section{M4R function in learning and motivation - can we really tell?}

Interestingly, the M4-D1R KO mice showed a high level of flexibility in adapting to the 5CSRTT tasks, which is unlikely to be caused by motivational drive alone. Crespo and colleagues observed increased levels of acetylcholine in the striatum equivalent to the level of acquisition in the operant runway, and hence suggested that acetylcholine in the striatum could function as a motivation learning signal (Crespo et al., 2006). The results from M4R-ChATCre mice support this idea. Deletion of the M4 receptor from cholinergic neurons rendered mice unable to acquire any learning task with the exception of food-enforced Pavlovian conditioning. These results clearly suggest that M4R-ChAT knockout mice are impaired in learning positive reinforcement behaviors. An explanation could be that in the absence of M4Rs from cholinergic interneurons, synaptic acetylcholine levels will reach a threshold where learning no longer occurs.

Unfortunately, it cannot be concluded from our results, whether the inability of M4R-ChAT knockout mice to respond to positive reinforcement is due to changes in motivational neurocircuitry or a learning-deficit occurring elsewhere in the brain. The absence of effects on cocaine induced locomotor sensitization and expression of immediate early genes, would indicate that the M4R-ChAT phenotype is less likely to be a direct consequence of altered dopaminergic signaling. 
Therefore, a clear limitation of this study is the lack of circuitry information. It would have been relevant to find the specific populations of M4R-ChAT neurons and M4R-D1RCre neurons responsible for the motivational effects. Unfortunately, this could not be done utilizing the Cre-viral strategy we employed in other studies. The Cre-virus mediated knockout would not have allowed us to distinguish between the D1R and Chat receptors pools, since both receptors are present within the same synapse. In the best-case scenario, one of the two phenotypes would have dominated. According to studies on the global M4R knockout mouse the dominant phenotype would likely have been the D1R-phenotype. Regardless, we would not have been able to conclude which receptor would be responsible for the specific behavior. Nevertheless, our results are in accordance with the notion that influence of acetylcholine on reinforcement learning follows a bell-shaped curve, where too little or too much transmitter in the synapse is unfavorable (Grasing, 2016). Our data suggests that during learning of positive reinforcement, acetylcholine release needs to be precisely tuned at the synaptic level. Loss of postsynaptic inhibition of D1Rneurons seems to contribute to an obsessive-compulsive phenotype, whereas a loss of presynaptic feedback inhibition causes learning deficits.

Our findings are according to the idea that neurocircuitry changes (i.e. sensitization) involved in encoding salience can lead to a pathologic addiction-like phenotype. This notion would support the theory of incentive sensitization. During normal circumstances the M4Rs on D1R neurons may constitute a functional brake on reinforcement learning. If $\mathrm{M} 4 \mathrm{R}$ expression is compromised in motivational neurocircuitry, such as the direct pathway neurons, this leads to a sensitized drugseeking phenotype. M4Rs on D1R neurons exert control over a broad array of behaviors related to addiction pathology, including locomotor sensitization, impulsivity, drug-seeking and relapse behavior after extinction. In line with this notion, a recent study on genetic polymorphisms within the M4R gene revealed a link to both cocaine and heroin addiction in human subjects (Levran et al., 2016). 


\section{CONCLUDING REMARKS AND PERSPECTIVES}

\section{Immune-to-brain signaling - universal principles and critical hubs}

The most exciting conclusion from the inflammation studies is that there exists a broadly utilized mechanism for signaling across the blood-brain-barrier, in which Myd88 functions as a universal signal-mediator for several cytokines and PAMPs. This function is generalizable to an extent that it is not only utilized by bacterial and viral PAMPs, but also directly by cytokines. Together with the findings from Blank and colleagues (2016), this thesis demonstrates a new path through which the immune system communicates to the brain and signal negative affect.

The BBB-endothelial signal transduction allows the innate immune system to subjugate several circuitries in different brain-areas and transmit a broad signal, changing physiological functions leading to anorexia, fever, inactivity, aversion and negative affective state. This happens naturally during infections and long-term inflammatory diseases, but it may also occur as a consequence of missignaling leading to cytokine production in psychopathologies. Fever is not a common symptom of major depressive disorder or of other affective disorders, which may indicate that specific cytokine routes are involved in the pathology of depression. As an example, one of the major cytokines upregulated during major depression, TNF $\alpha$, is considered controversial in the pyrogenic response.

Moreover, it could be relevant to distinguish between effects of peripheral versus central cytokines influencing affective state. An interesting study has shown that suicidal ideation directly correlates with IL-6 levels in cerebrospinal fluid of patients with major depressive disorder (Lindqvist et al., 2009). Nevertheless, the function of central cytokines in affective signaling remains to be fully explored.

In the present work, we uncovered how peripheral cytokines can mediate a central PGE2-signal directly influencing the activity of motivational neurocircuitry (Study I, II and III). In this context, our preliminary results suggest that the next step is to characterize the role of microglia in aversive and negative affective signaling. New studies have emerged demonstrating that microglia may be the critical hub in a broad variety of inflammation-mediated neuropathology, ranging from neurological disorders to psychopathologies (Atwells et al., 2017; Sampson et al., 2016; Setiawan et al., 2015). If there ever was a magic bullet this could be it...

In conclusion, the present studies have provided an array of new targets for the treatment of acute aversive conditions arising from systemic inflammation. These targets include: COX1, mPGES1, EP1R and EP2R, IFN- $\gamma \mathrm{R}$ and Cxcr3 together with 
the versatile MC4R (also a potential magic bullet). These targets will hopefully prove themselves useful in future studies on chronic inflammatory conditions and diseases.

\section{Dopaminergic neurons in motivation and negative affect - controversies and new perspectives}

An interesting feature of reward and aversion, demonstrated in the present work, is the involvement of many different molecular mechanisms regulating striatonigral and mesolimbic dopamine transmission. The presented biological pathways follow a concept of all paths lead to Rome. However, the specific midbrain neuropopulations targeted by these pathways remain to be further explored. Our findings indicate that different pathways in aversion and reward regulate activity of specific subpopulations of dopamine neurons. As mentioned, Lammel and colleagues (2012) elegantly demonstrated the concept of functionally distinct subpopulations of dopamine neurons, which has also been demonstrated by others (Bromberg-Martin et al., 2010). The presence of functionally distinct dopamine populations is an intriguing concept that is likely to explain differences observed in dopamine changes between MC4R knockout and controls during inflammation in study III, as well as in D1R-neuron-related phenotypes in study I, III and IV.

The classical hypothesis of striatal direct and indirect pathways implies that striatal D2R neurons are responsible for signaling aversion and avoidance, while the D1R expressing neurons are driving motivated behaviors and movement. Results from study I and III suggested exceptions to this rule, as excitable EP1Rs and MC4Rs on striatal D1R-neurons signal aversion by decreasing dopamine levels in specific target areas of the brain. In line with this, there exist some D1R expressing MSNs targeting dopamine neurons directly, instead of via GABAergic interneurons (Bocklish et al., 2013; Tanaka et al., 2009). The EP1R and MC4R expressing neurons responsible for signaling aversion could belong to this population. The age has finally come when modern circuit-tools will allow us to identify the dichotomies of direct- and in-direct pathway circuitry. This work has already begun (Kupchik et al., 2015) and will hopefully provide a more detailed understanding of the striatal neuropopulations in motivation and affective state in the future.

But where do these considerations leave us in terms of the concrete treatment of affective diseases? Targeting sub-populations of neurons can hardly be considered a realistic treatment option - or can it?

New treatment strategies for brain-area specific targeting have emerged; some of these are based on optogenetic circuit studies (Creed et al., 2015 and Chen et al., 2013; Terrano et al., 2015). As a successful example, a clinical study revealed that 
transcranial magnetic-stimulation of frontal brain areas is a feasible strategy for treating drug cravings and preventing drug consumption (Terrano et al., 2015). In addition, strategies are currently being developed for specific gene targeting, such as AAV-based therapies (Corti et al., 2017; Le Meur et al., 2017). Furthermore, conditional and area specific gene-targeting mouse models provide important information on biological dichotomies that may help to clarify divergent effects arising from pharmacological treatments.

The results from this thesis follow the general principle of the biphasic rewardprediction error, where increased dopamine levels signal reward, while decreases trigger aversion. Yet, according to our results, and the results of many others, the clear separation between striatonigral and mesolimbic circuitry needs to be revised, with an emphasis on defining specific functions of sub-circuitry.

Though it is clear that overlaying striatonigral and mesolimbic neurocircuitry define the encoding of positive and negative motivational valence, it was surprising to uncover exactly how close these processes are connected. The findings made in study III demonstrated how a single population of neurons in the striatum determines our perception of valence arising from specific stimuli. Our findings indicate that MC4Rs on D1R-neurons assign negative valence to salient stimuli, via regulation of dopamine tone, giving them a role as gatekeepers hindering aversive signals from inducing reward. This discovery illustrates how perception of "good and bad" can be determined by the signaling of a single neuropeptide, and thereby signifies exactly how intricately linked these mental phenomena are. Essentially, reward and aversion are two sides of the same coin.

Finally, the projects have raised the question of how motivation and affect relate to each other. It is broadly accepted that these two brain-processes are encoded by different neurocircuitry (i.e. dopamine versus serotonin, endogenous opioids, etc.) but also that they are conceptually interrelated. For instance, it is difficult to imagine states that lead to aversion without a negative affective component, yet it has been established that avoidance behavior is encoded by the motivational circuitry. There must exist a biological interface between motivation and affect. Future studies identifying this neurocircuitry will bring relevant information that may help us to find the underlying issues of affective disorders involving motivational disturbances, such as major depression and drug addiction. 


\section{ACKNOWLEDGEMENTS}

\section{Associate professor David Engblom, my supervisor}

It all started with the innocent little lie that Linköping is only a 2-hour drive away from Copenhagen.... And thank you for that - it has brought me so much. Thank you for all the interesting discussions, for being open minded and supporting me in pursuing my own ideas. You are the best PhD supervisor I could have imagined! But next time let's do less than 9 projects, right?

\section{Professor Anders Blomqvist, my other supervisor}

Thank you for the intellectual sparring - all the conversations I have had with you have been severely interesting. I have the deepest respect for your knowledge and analytical skills. You cut to the bone - that is truly inspiring.

\section{Assistant Professor Michael Fritz, the colleague}

You are the best teammate I could wish for. In the first years, you taught me everything about injections, place conditioning, stereotaxic surgeries and microdialysis. I don't know anyone who works as hard as you. The last 7 years has been a whirlwind of ideas, experiments and implementation of crazy techniques. I love to be caught in science with you - I can't imagine anything more interesting!

\section{Professor Markus Heilig}

Suddenly someone new walked through the door... And the time and space continuum expanded upon the entrance of a star. The horizon stretched and the world was never quit the same.... Thank you for being my mentor.

\section{Dr. Maarit Jaarola}

Who knew it would be possible to find a friend for life in this dark and deserted place? I am really thankful to know you. Thank you for all the interesting conversations and laughs about the absurdity of academia and life. Oh, and thank you for all the help with assays, microdissections, qPCR and genotyping and much more - you have been a cornerstone in the projects! Please come and visit me in California, so we can play "gin and rummy" in a sunnier place.

\section{Associate Professor Björn Granseth and his group, Sarah, Sofie and Erik}

Thank you for giving me the chance to learn how to record, sometimes a chance is all it takes... Thank you Sofie and Sarah for always being helpful. Thank you Erik, for being part of our summer course, the course wouldn't have been the same success without you. 


\section{Dr. Hugo Tejeda, NIDA.}

Thank you for all those hours you spend, supervising me to get the e-phys recordings just right. You taught me the details of the craft and it has already changed my life. I am forever grateful.

Professor Fredrik Elinder and his group-members, Johan, Urban, Sara, Malin, Jakob, Nina...

Thank you for welcoming me to the LiU's secret society of e-phys (despite my onoff participation in the electro-JCs) - it really meant a lot to me. In particular, thank you Urban for help with the recordings, your rig is fabulous; and thank you Johan for giving me a chance to teach in the Neurobiology course, this has really meant a lot to me. Finally, thank you Malin for being part of our summer course, we couldn't have done it without you.

Especially thanks to all you who made the summer-course in addiction biology a success (both 2016 and 2017)

Ida van der Woude, David Engblom, Pádraig D’Arcy, Markus Heilig, Per Jensen, Annika Thorsell, Anna Nilsson, Eric Johanson, Malin Silverå Ejneby, Riccardo Barchiesi, Esi Domi - you made the students learn a lot while keeping them happy. What an achievement! The course would not have been a success without your efforts.

\section{Professor Anders Fridberger and Dr. Pierre Hakizimana}

Thank you for the opportunity to teach and for being such great course organizers.

Thank you to the Engblom and Blomqvist groups for collaborations and teamwork

Anna Nilsson, Daniel Björk Wilhelms, Anand Kumar Singh, Carl Fredrik Rådberg,

Elahe Mirrasekhian, Andreas Löfberg, Joanna Zajdel, Kiseko Shionoya, Unn Kugelberg, Anna Eskilsson, Adriano Zagar, Filip Petkovic.

Thank you to all the people who have enriched my time at the $11^{\text {th }}$ and $12^{\text {th }}$ floor You know who you are, so if I forget anyone please do not feel sad about it, this thesis was written under a lot of stress. Thank you to neighbor-group members and other friendly people: Asif Aziz, Ayman Osman, Paul Hamilton, Elvar Theodorsson, Daniel Nätt, Estelle Barbier, Esi Domi, Riccardo Barchiesi, Gaëlle and Eric Augier, Li Xu, India Morrison, Lovisa Holm, Alison Atkins, Ann-Charlotte Johansson, Anna Asratian, Leah Mayo-Murphy, Simin Mohseni, Anita Öst, Peter Strålfors and many more. 


\section{Björn and Gabriel, and Ida and Pádraig.}

The summer course has brought so much joy, but the best part has really been to make new friends like you.

\section{My friends Puk, Cecilie, Maria, Bjarne and Tobias}

Thank you for not forgetting me, even though 3 years turned into 6. You enrich my life and keep me going whenever I feel that academia is getting the better of me... Thank you for being there.

\section{Mor - Ida}

You are my inspiration and support. Thank you for being so clever, strong and independent - and so incredibly creative and unpredictable! With you there is always a solution to everything - no matter the situation. Thanks to you, I will never run out of ideas...

\section{Michael}

Words cannot describe my love for you, nor my gratitude that you are in my life. You are my soulmate and I am so happy to have found you!

"Efter at have undersøgt over 100 siders koncentreret kritik har jeg ikke voeret $i$ stand til at finde argumenter, som anfoegter hovedindholdet $i$ min afhandling. I denne situation kan man så spørge, om jeg mon har studeret kritikken grundigt nok, eller om der evt. kan vere noget, jeg har overset. Jeg kan dog ikke se rettere, end at jeg har rørt ved alle veesentlige punkter. Nogle vil nok vare uenige med mig ... For mit eget vedkommende mener jeg dog ikke p.t. at kunne nå videre med sagen. Jeg vil derfor formode, at grunden til, at jeg ikke kan finde nogen afgørende argumenter mod min teori om jeg'ets varensform, er, at der ikke er nogen, og at dette igen skyldes, at en korrekt opfattelse ikke på korrekt vis kan gendrives"

Erich Klawonn, Jegets ontologi 


\section{REFERENCES}

Alcami P, Franconville R, Llano I, Marty A. 2012. Measuring the firing rate of high-resistance neurons with cell-attached recording. The Journal of Neuroscience 32(9): 3118-3130.

Alexander GM, Rogan SC, Abbas AI, Armbruster BN, Pei Y, Allen JA, Nonneman RJ, Hartmann J, Moy SS, Nicolelis MA, McNamara JO, Roth BL. Remote control of neuronal activity in transgenic mice expressing evolved G protein-coupled receptors. Neuron 63: 27-39.

Anrather J, Gallo EF, Kawano T, Orio M, Abe T, Gooden C, Zhou P, Iadecola C. 2011. Purinergic signaling induces cycloxygenase-1-dependent prostanoid synthesis in microglia: roles in the outcome of excitotoxic brain injury. PloS One 6(10): e25916.

Aosaki T, Tsubokawa H, Ishida A, Watanabe K, Graybiel AM, Kimura M. 1994. Responses of tonically active neurons in the primate's striatum undergo systematic changes during behavioral sensorimotor conditioning. The Journal of Neuroscience 14(6): 3969-3984.

Aponte Y, Atasoy D, Sternson SM. 2011. AGRP neurons are sufficient to orchestrate feeding behavior rapidly without training. Nature Neuroscience 14: 351-355.

Aschauer DF, Kreuz S, Rumpel S. 2013. Analysis of Transduction efficiency, tropism and axonal transport of AAV serotypes 1, 2, 5, 6, 8 and 9 in the mouse brain. PLOS One 8(9): 1-16.

Atasoy D, Aponte Y, Su HH, Sternson SM. 2008. A FLEX switch targets channelrhodopsin-2 to multiple cell types for imaging and long-range circuit mapping. The Journal of Neuroscience 28: 70257030 .

Atasoy D, Betley JN, Su HH, Sternson SM. 2012. Deconstruction of a neural circuit for hunger. Nature 488: 172-177.

Atasoy D, Betley JN, Li WP, Su HH, Sertel SM, Scheffer LK, Simpson JH, Fetter RD, Sternson SM. 2014. A genetically specified connectomics approach applied to long-range feeding regulatory circuits. Nature Neuroscience 17(12): 1830-1839.

Attwells S, Setiawan E, Wilson AA, Rusjan PM, Mizrahi R, Miler L, Xu C, Richter MA, Kahn A, Kish SJ, Houle S, Ravindran L, Meyer JH. 2017. Inflammation in the Neurocircuitry of ObsessiveCompulsive Disorder. JAMA Psychiatry 74(8): 833-840.

Arrigoni E, Saper CB. 2014. What optogenetic stimulation is telling us (and failing to tell us) about fast neurotransmitters and neuromodulatores in brain circuits for wake-sleep regulation. Current Opinion in Neurobiology 29: 165-171.

Banks WA, Kastin AJ, Broadwell RD. 1995. Passage of cytokines across the blood-brain barrier. Neuroimmunomodulation 2(4): 241-248.

Banks WA. 2015. Peptides and the blood-brain barrier. Peptides 72: 16-19.

Banner DW, D'arcy A, Janes W, Gentz R, Schoenfeld HJ, Broger C, Loetscher H, Lesslauer W. 1993. Crystal structure of the soluble human $55 \mathrm{kd}$ TNF receptor-human TNF beta complex: implications for TNF activation. Cell 73(3): 431-445.

Bardo MT, Rowlett JK, Harris MJ. 1995. Conditioned place preference using opiate and stimulant drugs: a meta-analysis. Neuroscience and biobehavioral reviews 19(1): 39-51. 
Bear MF, Connors BW, Paradiso MA. 2007. Neuroscience - Exploring the brain, Third edition. Lippincott Williams and Wilkins, a Wolters Kluwer Business.

Belin D and Everitt BJ. 2008. Cocaine seeking habits depend upon dopamine-dependent serial connectivity linking the ventral with the dorsal Striatum. Neuron 67: 432-441.

Benoit SC, Sheldon RJ, Air E, Messerschmidt P, Wilmer KA, Hodge KMB, Jones MB, Eckstein DMM, McOsker CC, Woods SC, Seeley RJ. 2003. Assesment of the aversive consequences of acute and chronic administration of the melanocortin agonist MTII. International Journal of Obesity 27: 550-556. Berridge KC and Robinson TE. 1998. What is the role of dopamine in reward: hedonic impact, reward learning or incetive salience? Brain research reviews 28: 309-369.

Betley JN, Cao ZFH, Ritola KD, Sternson SM. 2013. Parallel, redundant circuit organization for homeostatic control of feeding behavior. Cell 155(6): 1337-1350.

Betley JN, Xu S, Cao ZFH, Gong R, Magnus CJ, Yu Y, Sternson SM. 2015. Neurons for hunger and thirst transmit a negative-valence teaching signal. Nature 14(521): 180-185.

Bevan P, Cools AR, Archer T. 1989. Behavioural Pharmacology of 5-HT. Library of congress cataloging in publication data. Lawrence Erlbaum Associates, Inc, Publishers.

Biesmans S, Meert TF, Bouwknech JA, Acton PD, Davoodi N, De Haes P, Kuijlaars J, Langlois X, Matthews LJR, Ver Donck L, Hellings N, Nuydens R. 2013. Systemic immune activation leads to neuroinflammation and sickness behavior in Mice. Mediators of inflammation 2013: 1-14.

Blank T, Detje CN, Spiess A, Hagemeyer N, Brendecke SM, Wolfart J et al. 2016. Brain Endothelialand Epithelial-specific Interferon Receptor Chain 1 drives virus-induced sickness behavior and cognitive impairment. Immunity 44(4): 901-912.

Bluthé RM, Layé S, Michaud B, Combe C, Dantzer R, Parnet P. 2000. Role of interleukin-1 $\beta$ and tumor necrosis factor- $\alpha$ in lipopolysaccharide-induced sicknes behavior: a study with interleukin-1 type I receptor-deficient mice. European Journal of Neuroscience 12: 4447-4456.

Bocklisch C, Pascoli V, Wong JCY, House DRC, Yvon C, de Roo M, Tan KR, Lüscher C. 2013. Cocaine Disinhibits Dopamine Neurons by Potentiation of GABA Transmission in the Ventral Tegmental Area. Science 341: 1521-1525.

Bouchard LC, Antoni MH, Blomberg BB, Stagl JM, Gudenkauf LM, Jutagir DR, Diaz A, Lechner S, Glück S, Derhagopian RP, Carver CS. 2016. Postsurgical Depressive Symptoms and Proinflammatory Cytokine Elevations in Women undergoing Primary Treatment for Breast Cancer. Psychosomatic Medicine 78(1): 26-37

Brabant C, Quertemont E and Tirelli E. 2005. Influence of the dose and the number of drug-context pairings on the magnitude and the long-lasting retention of cocaine-induced conditioned place preference in C57BL/6J mice. Psychopharmacology 180(1): 33-40.

Brady ST, Siegel GJ, Albers RW, Price DL. 2012. Basic neurochemistry: Principles of Molecular, Cellular, and Medical Neurobiology ( $8^{\text {th }}$ edition). Elsevier Academic Press, Oxford, United Kingdom.

Braida D, Paladini E, Griffini P, Lamperti M, Maggi A, Sala M. 1996. An inverted U-shaped curve for heptylphysostigmine on radial maze performance in rats: comparison with other cholineesterase inhibitiors. European Journal of Pharmacology 302:13-20. 
Braida D, Paladini E, Griffini P, Lamperti M, Colibretti L, Sala M. 1997. Long-lasting antiamnesic effect of a novel anticholinesterase inhibitor (MF268). Pharmacology, Biochemistry and Behavior 59(4):897-901.

Branda CS and Dymecki SM. 2004. Talking about a revolution: The impact of site-specific recombinases on genetic analysis in mice. Developmental Cell 6: 7-28.

Britt JP, Benaliouad F, McDevitt RA, Stuber GD, Wise RA, and Bonci A. 2012. Synaptic and behavioral profile of multiple glutamatergic inputs to the nucleus accumbens. Neuron: 76(4):790-803. Bromberg-Martin ES, Matsumoto M, Hikosaka O. 2010. Dopamine in motivational control: Rewarding, Aversive, and Alerting. Neuron (68): 815-834.

Brydon L, Harrison NA, Walker C, Steptoe A, Critchley HD. 2008. Peripheral inflammation is associated with altered substantia nigra activity and psychomotor slowing in humans. Biological Psychiatry 63(11): 1022-1029.

Cachope R, Mateo Y, Mathur BN, Irving J, Wang HL, Morales M, Lovinger DM, Cheer JF. 2012. Selective activation of cholinergic interneurons enhances accumbal phasic dopamine release: setting the tone for reward processing. Cell Rep 2:33-41.

Candelario-Jalil E, Slawik H, Ridelis I, Waschbisch A, Akundi RS, Hüll M, Fiebich BL. 2005. Regional distribution of the prostaglandin E2 receptor EP1 in the rat brain: accumulation in Purkinje cells of the cerebellum. Journal of Molecular Neuroscience 27(3): 303-310.

Castle MJ, Gershenson ZT, Giles AR, Holzbauer ELF, Wolfe JH. 2014. Adeno-Associated virus serotypes 1, 8, and 9 share conserved mechanisms for anterograde and retrograde axonal transport. Human Gene Therapy 25: 705-720.

Cao C, Matsumura K, Yamagata K, Watanabe Y. 1995. Induction by lipopolysaccharide of cyclooxygenase-2 mRNA in rat brain; its possible role in the febrile response. Brain Research 697(1-2): 187-196.

Chandrasekharan S, Foley NA, Jania L, Clark P, Audoly LP, Koller BH. 2005. Coupling of COX-1 to mPGES1 for prostaglandin E2 biosynthesis in the murine mammary gland. The Journal of Lipid Research 46(12): 2636-2648.

Chen BT, Yau HJ, Hatch C, Kusumoto-Yoshida I, Cho SL, Hopf FW, Bonci A. 2013. Rescuing cocaine-induced prefrontal cortex hypoactivity prevents compulsive cocaine seeking. Nature 496(7445): 359-362.

Chen CJ, Kono H, Golenbock D, Reed G, Akira S, Rock KL. 2007. Identification of a key pathway required for the sterile inflammatory response triggered by dying cells. Nature Medicine 13(7): 851856.

Childs EL, de Wit H. 2009. Amphetamine induced place preference in humans. Biological Psychiatry 65(10): 900-904.

Ching S, Zhang H, Belevych N, He L, Lai W, Pu XA, Jaeger LB, Chen Q, Quan N. 2007. Endothelialspecific knockdown of interleukin-1 (IL-1) type 1 receptor differentially alters CNS responses to IL-1 depending on its route of administration. The Journal of Neuroscience 27(39): 10476-10486. 
Corti M, Liberati C, Smith BK, Lawson LA, Tuna IS, Conlon TJ, Erger KE, Islam S, Herzog R, Fuller DD, Collins S, Byrne BJ. 2017. Safety of Intradiaphragmatic Delivery of Adeno-Associated VirusMediated Alpha-Glucosidase (rAAV1-CMV-hGAA) Gene Therapy in children affected by Pompe Disease. Human Gene Therapy Clinical Development doi: 10.1089/humc.2017.146. (ahead of print).

Creed M, Pascoli VJ, Lüscher C. 2015. Addiction therapy. Refining deep brain stimulation to emulate optogenetic treatment of synaptic pathology. Science 347(6222): 659-664.

Crespo JA, Sturm K, Saria A, Zernig G. 2006. Activation of Muscarinic and Nicotinic Acetylcholine Receptors in the Nucleus Accumbens Core Is Necessary for the Acquisition of Drug Reinforcement. The Journal of Neuroscience 26(22): 6004-6010.

Crespo JA, Stöckl P, Zorn K, Saria A, Zernig G. 2008. Nucleus accumbens core acetylcholine is preferentially activated during acquisition of drug- vs food-reinforced behavior. Neuropsychopharmacology 33(13): 3213-3220.

Copeland NG, Jenkins NA, Court DL. 2001. Recombineering: A powerful new tool for mouse functional genomics. Nature reviews genetics 2: 769-779.

Croft M, Siegel RM. 2017. Beyond TNF: TNF superfamily cytokines as targets for the treatment of rheumatic diseases. Nature Reviews Rheumatology 13(4): 217-233.

Cui H, Mason BL, Lee C, Nishi A, Elmquist JK, Lutter M. 2012. Melanocortin 4 receptor signaling in dopamine 1 receptor neurons is required for procedural memory learning. Physiology and Behavior 106(2): 201-210.

Cui H, Lutter M. 2013. The expression of MC4Rs in D1R neurons regulate food intake and locomotor sensitization to cocaine. Genes, Brain, and Behavior 12(6): 658-665.

Cunningham CL and Niehus JS. 1993. Drug-induced hypothermia and conditioned place aversion. Behavioral Neuroscience 107(3): 468-479.

Dalley JW, McGaughy J, O’Connell MT, Cardinal RN, Levita L, Robbins TW. 2001. Distinct changes in cortical acetylcholine and noradrenaline efflux during contingent and noncontingent performance of a visual attention task. The Journal of Neuroscience 21(13): 4908-4914.

Daniels D, Patten CS, Roth JD, Yee DK, Fluharty SJ. 2003. Melanocortin receptor signaling through mitogen-activated protein kinase in vitro and in rat hypothalamus. Brain Research 986: 1-11.

Dantzer R. 2001. Cytokine-induced sickness behavior: where do we stand? Brain, Behavior and Immunity 15(1): 7-24.

Dantzer R, O’Connor JC, Freund GG, Johnson RW, Kelley KW. 2008. From inflammation to sickness and depression: when the immunesystem subjugates the brain. Nature Reviews Neuroscience 9(1): 4656.

Dantzer R. 2017. Role of the Kynurenine Metabolism Pathway in Inflammation-Induced Depression: Preclinical Approaches. Current Topics in Behavioral Neuroscience. 31: 117138.

Dautan D, Huerta-Ocampo I, Witten IB, Deisseroth K, Bolam JP, Gerdjikov T, Mena-Segovia J. 2014. A Major External Source of Cholinergic Innervation of the Striatum and Nucleus Accumbens Originates in the Brainstem. The Journal of neuroscience 34(13): 4509-4518. 
Dinarello CA. 2000. The role of the interleukin-1-receptor antagonist in blocking inflammation mediated by interleukin-1. The New England Journal of Medicine 343(10): 732-734.

De la Cour C, Sörensen G, Wortwein G, Weikop P, Dencker D, Fink-Jensen A, Molander A. 2015. Enhanced self-administration of alcohol in muscarinic acetylcholine $\mathrm{M}_{4}$ receptor knockout mice. European Journal of Pharmacology 746: 1-5.

Delfs JM, Zhu Y, Druhan JP, and Aston-Jones G. 2000. Noradrenaline in the ventral forebrain is critical for opiate withdrawal-induced aversion. Nature 403(6768):430-4.

Doetschman T, Gregg RG, Maeda N, Hooper ML, Melton DW, Thompson S, Smithies O. 1987. Targeted correction of a mutant HPRT gene in mouse embryonic stem cells. Nature 330(6148): 576578.

Dowlati Y, Herrmann N, Swardfager W, Liu H, Sham L, Reim EK, Lanctôt KL. 2010. A meta-analysis of cytokines in major depression. Biological Psychiatry 67(5): 446-457.

Ehrich JM, Messinger DI, Knakal CR, Kuhar JR, Schattauer SS, Bruchas MR, Zweifel LS, Kieffer BL, Phillips PEM, Chavkin C. 2015. Kappa opioid receptor-induced aversion requires p38 MAPK activation in VTA dopamine neurons. The Journal of Neuroscience 35(37): 12917-12931.

Eisenberger NI, Berkman ET, Inagaki TK, Rameson LT, Mashal NM, Irwin MR. 2010. Inflammation induced anhedonia: endotoxin reduces ventral striatum responses to reward. Biological Psychiatry 68(8):748-754.

Ek M, Kurosawa M, Lundeberg T, Ericsson A. 1998. Activation of vagal afferents after intravenous injection of interleukin-1beta: role of endogenous prostaglandins. Journal of Neuroscience 18(22): 9471-9479.

Ek M, Arias C, Sawchenko P, Ericsson-Dahlstrand A. 2000. Distribution of the EP3 Prostaglandin E2 receptor subtype in the rat brain: Relationship to sites of interleukin-1-induced cellular responsiveness. The Journal of Comparative Neurology 428: 5-10.

Ek M, Engblom D, Saha S, Blomqvist A, Jakobsson PJ, Ericsson-Dahlstrand A. 2001. Inflammatory response: pathway across the blood-brain barrier. Nature 410(6827): 430-431.

Elander L, Engström L, Ruud J, Mackerlova L, Jakobsson PJ, Engblom D, Nilsberth C, Blomqvist A. 2009. Inducible prostaglandin E2 synthesis interacts in a temporally supplementary sequence with constitutive prostaglandin-synthesizing enzymes in creating the hypothalamic-pituitary-adrenal axis response to immune challenge. The Journal of Neuroscience 29(5): 1404-1413.

Engblom D, Saha S, Engström L, Westman M, Audoly LP, Jakobsson PJ, Blomqvist A. 2003. Microsomal prostaglandin E synthase-1 is the central switch during immune-induced pyresis. Nature Neuroscience 6(11): 1137-1138.

Eskilsson A, Matsuwaki T, Shionoya K, Mirrasekhian E, Zajdel J, Schwaninger M, Engblom D, Blomqvist A. 2017. Immune-Induced Fever Is Dependent on Local But Not Generalized Prostaglandin E2 Synthesis in the Brain. The Journal of Neuroscience 37(19): 5035-5044.

Eskilsson A, Tachikawa M, Hosoya K, Blomqvist A. 2014. Distribution of microsomal prostaglandin E synthase-1 in the mouse brain. Journal of Comparative Neurology 522(14): 3229-3244. 
Estevez J, Chen VL, Podlaha O, Li B, Le A, Vutien P, Chang ET, Rosenberg-Hasson Y, Jiang Z, Pflanz S, Ge D, Gaggar A, Nguyen MH. 2017. Differential serum cytokine profiles in patients with chronic hepatitis B,C, and hepatocellular carcinoma. Scientific Reports 7: 1-11.

Ettenberg A, Raven MA, Danluck DA, Necessary BD. 1999. Evidence for opponent-process actions of intravenous cocaine. Pharmacology, Biochemistry, and Behavior 64(3): 507-512.

Ettenberg A. 2009. The runway model of self-administration. Pharmacology, biochemistry, and behavior 91(3): 271-277.

Farooqi IS, Keogh JM, Yeo GS, Lank EJ, Cheetham T, O’Rahilly S. 2003. Clinical spectrum of obesity and mutations in the melanocortin 4 receptor gene. The New England Journal of Medicine 348(12): 1085-1095.

Felger JC. 2017. The role of dopamine in inflammation-associated depression: mechanisms and therapeutic implications. Current topics in behavioral neurosciences 31: 199-219.

Fenno L, Yizhar O, Deisseroth K. 2011. The development and application of optogenetics. Annual review of neuroscience 34: 389-412.

Fritz M, Klement S, El Rawas R, Saria A, Zernig G. 2011. Sigma1 receptor antagonist BD1047 enhances reversal of conditioned place preference from cocaine to social interaction. Pharmacology 87(1-2): 45-48.

Gantz I, Miwa H, Konda Y, Shimoto Y, Tashiro T, Watson SJ, DelValle J, Yamata T. 1993. The Journal of Biological Chemistry 268(20): 15174-15179.

Gardner RL, Brook FA. 1997. Reflections on the biology of embryonic stem cells. International Journal of Developmental Biology 41: 235-243.

García-Bueno B, Serrats J, Sawchenko PE. 2009. Cerebrovascular cyclooxygenase-1 expression, regulation, and role in hypothalamic-pituitary-adrenal axis activation by inflammatory stimuli. The Journal of Neuroscience 29(41): 12970-12981.

Gauffin K, Vinnerljung B, Fridell M, Hesse M, Hjern A. 2013. Childhood socio-economic status, school failure and drug abuse: a Swedish national cohort study. Addiction 108(8): 1441-1449.

Geiger BM, Frank LE, Caldera-Siu AD, Pothos EN. 2008. Survivable stereotaxic surgery in rodents. Journal of visualized experiments 20: 880 .

Ghamari-Langroudi M, Digby GJ, Sebag JA, Millhauser GL, Palomino R, Matthews R, Gillyard T, Panaro BL, Tough IR, Cox HM, Denton JS, Cone RD. 2015. G-protein independent coupling of MC4R to Kir7.1 in hypothalamic neurons. Nature 520: 94-98.

Glaser S, Anasrassiadis K, Stewart F. 2005. Current issues in mouse genome engineering. Nature Genetics 37: 1187-1193.

Goehler LE, Busch CR, Tartaglia N, Relton J, Sisk D, Maier SF, Watkins LR. 1995. Blockade of cytokine induced conditioned taste aversion by subdiaphragmatic vagotomy: further evidence for vagal mediation of immune-brain communication. Neuroscience Letters 185(3): 163-166.

Gomez JL, Bonaventura J, Lesniak W, Mathews WB. Sysa-Sha P, Rodriguez LA, Ellis RJ, Richie CT, Harvey BK, Dannals RF, Pomper MG, Bonci A, Michealides M. 2017. Chemogenetics revealed: DREADD occupancy and activation via converted clozapine. Science 357(6350): 503-507. 
Goyal SN, Kokare DM, Chopde CT, Subhedar NK. 2006. Alpha-melanocyte stimulating hormone antagonizes antidepressant-like effect of neuropeptide $\mathrm{Y}$ in Porsolt's test in rats. Pharmacology, Biochemistry, and Behavior 85(2): 369-377.

Grant KA, Shively CA, Nader MA, Ehrenkaufer RL, Line SW, Morton TE, Gage HD, Mach RH. 1998. Effect of social status on striatal dopamine D2 receptor binding characteristics in cynomolgus monkeys assessed with positron emission tomography. Synapse 29, 80-83.

Grasing K. 2016. A threshold model for opposing actions of acetylcholine on reward behavior: Molecular mechanisms and implications for treatment of substance abuse disorders. Behavioural Brain Research 312: 148-162.

Hannestad J, DellaGioia N, Bloch M. 2011. The effect of antidepressant medication treatment on serum levels of inflammatory cytokines: a meta-analysis. Neuropsychopharmacology 36(12): 2452-2459.

Haapakoski R, Mathieu J, Ebmeier KP, Alenius H, Kivimäki M. 2015. Cumulative meta-analysis of interleukin 6 and $1 \beta$, tumor necrosis factor $\alpha$ and C-reactive protein in patients with major depressive disorder. Brain, Behavior and Immunity 49: 206-215.

Heien MLAV, Khan AS, Ariansen JL, Cheer JF, Phillips PEM, Wassum KM, Wightman RM. 2005. Real-time measurement of dopamine fluctuations after cocaine in the brain of behaving rats. PNAS 102(29): 10023-10028.

Hellard EAR, Impastato RA, Gilpin NW. 2016. Intra-cerebral and intra-nasal melanocortin-4 receptor antagonist blocks withdrawal hyperalgesia in alcohol dependent rats. Addiction Biology 22(3): 692-701.

Hentges ST, Otero-Corchon V, Pennock RL, King CM, Low MJ. 2009. Proopiomelanocortin expression in both GABA and Glutamate neurons. The Journal of Neuroscience 29(43): 13684-13690.

Hersman S, Cushman J, Lemelson N, Wassum K, Lotfipour S, Fanselow MS. 2017. Optogenetic excitation of cholinergic inputs to hippocampus primes future contextual fear associations. Scientific reports 7(1): 1-6.

Hodes GE, Kana V, Menard C, Merad M, Russo SJ. 2015. Neuroimmune mechanisms of depression. Nature Neuroscience 18(10): 1386-1393.

Hoess RH, Wierzbicki A, Abremski K. 1986. The role of the loxP spacer region in P1 site-specific recombination. Nucleic Acids Research 14(5): 2287-2300.

Ilango A, Kesner AJ, Keller KL, Stuber GD, Bonci A, Ikemoto S. 2014. Similar roles of substantia nigra and ventral tegmental dopamine neurons in reward and aversion. The Journal of Neuroscience 34(3): 817-822.

Indra AK, Warot X, Brocard J, Bornert JM, Xiao JH, Chambon P, Metzger D. 1999, Temporally controlled site-specific mutagenesis in the basal layer of the epidermis: comparison of the recombinase activity of the tamoxifen-inducible Cre-ER(T) and CreER(T2) recombinases. Nucleic Acids Research 27: 4324-4327.

Jakobsson PJ, Thorén S, Morgenstern R, Samuelsson B. 1999. Identification of human prostaglandin E synthase: a microsomal, glutathione-dependent, inducible enzyme, constituting a potential novel drug target. PNAS 96(13): 7220-7225.

Jeon J, Dencker D, Wörtwein G, Woldbye DPD, Cui Y, Davis AA, Levey AI, Schütz G, Sager TN, Mörk A, Li C, Deng CX, Fink-Jensen A, Wess J. 2010. A Subpopulation of Neuronal $\mathrm{M}_{4}$ Muscarinic 
Acetylcholine Receptors Plays a Critical Role in Modulating Dopamine-Dependent Behaviors. The Journal of Neuroscience 30(6): 2396-2405.

Jiang L, Kundu R, Lederman JD, Lopex-Hernandez GY, Ballinger EC, Wang S, Talmage DA, Role LW. 2016. Cholinergic signaling controls conditioned fear behaviors and enhances plasticity of cortical amygdala circuits. Neuron 90: 1057-1070.

Johansen JP, Fields HL, Manning BH. 2001. The affective component of pain in rodents: Direct evidence for a contribution of the anterior cingulate cortex. PNAS 98(14): 8077-8082.

Joshua M, Adler A, Mitelman R, Vaadia E, Bergman H. 2008. Midbrain dopaminergic neurons and striatal cholinergic interneurons encode the difference between reward and aversive events at different epochs of probabilistic classical conditioning trials. The Journal of Neuroscience 28(45): 11673-11684.

Kalange AS, Kokare DM, Singru PS, Upadhya MA, Chopde CT, Subhedar NK. 2007. Central administration of the selective melanocrotin 4 receptor antagonist HS014 prevents morphine tolerance and withdrawal hyperalgesia. Brain Research 1181: 10-20.

Kappelmann N, Lewis G, Dantzer R, Jones PB, Khandaker GM. 2016. Antidepressant activity of anticytokine treatment: a systematic review and meta-analysis of clinical trials of chronic inflammatory conditions. Molecular Psychiatry: 1-9.

Kaster MP, Gadotti VM, Calixto JB, Santos AR, Rodrigues AL. 2012. Depressive-like behavior induced by tumor necrosis factor- $\alpha$ in mice. Neuropharmacology 62(1): 419-426.

Keiflin R and Janak PH (2015). Dopamine prediction errors in reward learning and addiction: from theory to neural circuitry. Neuron 88(2): 247-263.

Kindt TJ, Goldsby RA and Osborne J. 2007. Immunology. W.H. Freeman and Company, New York, USA.

Kishi T, Aschkenasi CJ, Lee CE, Mountjoy KG, Saper CB, Elmquist JK. 2003. Expression of Melanocortin 4 receptor mRNA in the central nervous system of the rat. The Journal of Comparative Neurology 457: 213-235.

Kitaoka S, Furuyashiki T, Nishi A, Shuto T, Koyasu S, Matsuoka T, Miyasaka M, Greengard P, Narumiya S. 2007. Prostaglandin E2 acts on EP1 receptor and amplifies both dopamine D1 and D2 receptor signaling in the striatum. The Journal of Neuroscience 27(47): 12900-12907.

Klawonn AM, Nilsson A, Rådberg CF, Lindström SH, Ericson M, Granseth B, Engblom D, Fritz M. 2017. The sigma-2 receptor selective agonist siramesine ( $\mathrm{Lu} 28-179)$ decreases cocaine-reinforced Pavlovian learning and alters glutamatergic and dopaminergic input to the striatum. Frontiers in Pharmacology. DOI:10.3389/fphar.2017.00714.

Kokare DM, Dandekar MP, Chopde CT, Subhedar N. 2005. Interaction between neuropeptide Y and alpha-melanocyte stimulating hormone in amygdala regulates anxiety in rats. 2005. Brain research 1043(2):107-114.

Koob GF and Le Moal M (2006). Neurobiology of addiction. Academic Press (DSM-IV on p4).

Koob GF, Le Moal. 2008. Neurobiological mechanisms for opponent motivational processes in addiction. Philosophical transactions B 363: 3113-3123.

Koob GF, Volkow ND. 2010. Neurocircuitry of addiction. Neuropsychopharmacology reviews 35: $217-$ 238. 
Koller BH, Hagemann LJ, Doetschman T, Hagaman JR, Huang S, Williams PJ, First NL, Maeda N, Smithies O. 1989. Germ-line transmission of a planned alteration made in a hypoxanthinephosphoribosyltransferase gene by homologous recombination in embryonic stemcells. PNAS 86(22): 8927-8931.

Krashes MJ, Lowell BB, Garfield AS. 2016. Melanocortin-4 receptor-regulated energy homeostasis. Nature Neuroscience 9(2): 206-219.

Kravitz AV, Kreitzer AC. 2012. Striatal mechanisms underlying movement, reinforcement, and punishment. Physiology 27: 167-177.

Kravitz AV, Tye LD, Kreitzer AC. 2012. Distinct roles for direct and indirect pathway striatal neurons in reinforcement. Nature Neuroscience 15(6): 816-818.

Kupchik YM, Brown RM, Heinsbroek JA, Lobo MK, Schwartz DJ, Kalivas PW. 2015. Coding the direct/indirect pathways by D1 and D2 receptors is not valid for accumbens projections. Nature Neuroscience 18(9): 1230-1232.

Kügler S, Kilic E, Bähr M. 2003. Human synapsin 1 gene promoter confers highly neuron-specific long-term transgene expression from an adenoviral vector in the adult rat brain depending on the transduced area. Gene therapy 10(4): 337-347.

Land BB, Bruchas MR, Lemos JC, Xu M, Melief EJ, Chavkin C. 2008. The dysphoric component of stress is encoded by activation of the dynorphin k-opioid system. The Journal of Neuroscience 28(2): 407-414.

Land BB, Bruchas MR, Schattauer S, Giardino WJ, Aita M, Messinger D, Hnasko TS, Palmiter RD, Chavkin C. 2009. Activation of the kappa opioid receptor in the dorsal raphe nucleus mediates the aversive effects of stress and reinstates drug seeking. PNAS 106(45): 19168-19173.

Langmead CJ, Watson J, Reavill C. 2008. Muscarinic acetylcholine receptors as CNS drug targets, Pharmacol. Ther. 117:232-243.

Lazarus M, Yoshida K, Coppari R, Bass CE, Mochizuki T, Lowell BB, Saper CB. 2007. EP3 prostaglandin receptors in the median preoptic nucleus are critical for fever responses. Nature Neuroscience 10(9): 1131-1133.

Lee G and Saito I. 1998. Role of nucleotide sequences of loxP spacer region in Cre-mediated recombination. Gene 216(1): 55-65.

Lee J, Finkelstein J, Choi JY, Witten IB. 2016. Linking Cholinergic Interneurons, Synaptic Plasticity, and Behavior during the Extinction of a Cocaine-Context Association. Neuron 90, 1071-1085.

Le Meur G, Lebranchu P, Billaud F, Adjali O, Schmitt S, Bézieau S, Péréon Y, Valabregue R, Ivan C, Darmon C, Moullier P, Rolling F, Weber M. 2017. Safety and Long-Term Efficacy of AAV4 Gene Therapy in Patients with RPE65 Leber Congenital Amaurosis. Molecular Therapy

DOI: http://dx.doi.org/10.1016/j.ymthe.2017.09.014 (ahead of print).

Lequin RM. 2005. Enzyme Immunoassay (EIA)/Enzyme-linked Immunosorbent Assay (ELISA). Clinical Chemistry 51(12): 2415-2418.

Levran O, Randesi M, Peles E, Correa da Rosa J, Ott J, Rotrosen J, Adelson M, Kreek MJ. 2016. African-specific variability in the acetylcholine muscarinic receptor M4: association with cocaine and heroin addiction. Pharmacogenomics 17(9): 995-1003. 
Liebregts T, Adam B, Bredack C, Röth A, Heinzel S, Lester S, Downie-Doyle S, Smith E, Drew P, Talley NJ, Holtman G. 2007. Immune activation in patients with irritable bowel syndrome. Gastroenterology 132(3): 913-920.

Lim BK, Huang KW, Grueter BA, Rothwell PE, Malenka RC. 2012. Anhedonia requires MC4Rmediated synaptic adaptations in nucleus accumbens. Nature 487(7406): 183-189.

Lindqvist D, Janelidze S, Hagell P, Erhardt S, Samuelsson M, Minthon L, Hansson O, Björkqvist M, Träskman-Bendz L, Brundin L. 2009. Interleukin-6 is elevated in the cerebrospinal fluid of suicide attempters and related to symptom severity. Biological Psychiatry 66(3): 287-292.

Liu C, Lee S, Elmquist JK. 2014. Circuits controlling energy balance and mood: Inherently intertwined or just complicated intersections? Cell metabolism 19(6): 902-909.

Liu J, Garza JC, Li W, Lu XY. 2013. Melanocortin-4 receptor in the medial amygdala regulates emotional stress-induced anxiety-like behavior, anorexia and corticosterone secretion. The International Journal of Neuropsychopharmacology 16(1): 105-120.

Liu H, Kishi T, Roseberry AG, Cai X, Montez JM, Friedman JM, Elmquist JK. 2003. Transgenic mice expressing green fluorescent protein under the control of the melanocortin-4 receptor promoter. The Journal of Neuroscience 23(18): 7143-7154.

Lodish H, Berk A, Kaiser CA et al. 2008. Molecular cell biology sixth edition. W.H. Freeman and Company.

Lotrich FE. 2009. Major Depression during interferon- $\alpha$ treatment: vulnerability and prevention. Dialogues in Clinical Neuroscience 11(4): 417-425.

Lu XY, Barsh GS, Akil H, Watson SJ. 2003. Interaction between alpha-melanocyte-stimulating hormone and corticotropin-releasing hormone in the regulation of feeding and hypothalamo-pituitaryadrenal responses. The Journal of Neuroscience 23(21): 7863-7872.

Lüscher C, Malenka RC. 2011. Drug-evoked synaptic plasticity in addiction: From molecular changes to circuit remodeling. Neuron 69(4): 650-663.

Maes JH and Vossen JM. 1993. Context conditioning: positive reinforcing effects of various foodrelated stimuli. Physiology and Behavior 53(6): 1227-1229.

Mahmoud ME, Fereig R, Nishikawa Y (2017). Involvement of host defense mechanisms against Toxoplasma gondii infection in anhedonic and despair-like behaviors in mice. Infection immunology

Majumder S, Zhou LZ, Chaturvedi P, Babcock G, Aras S, Ransohoff RM. 1998. p48/STAT-1alphacontaining complexes play a predominant role in induction of IFN-gamma-inducible protein, $10 \mathrm{kDa}$ (IP-10) by IFN-gamma alone or in synergy with TNF-alpha. Journal of Immunology 161(9): 4736-4744.

Marks DL, Ling N, Cone RD. 2001. Role of the central melanocortin system in cachexia. Cancer Research 61(4): 1432-1438.

Martinez D, Broft A, Foltin RW, Slifstein M, Hwang DR, Huang Y, Perez A, W Gordon Frankel, Thomas Cooper, Herbert D Kleber, Marian W Fischman, Laruelle M. 2004. Cocaine Dependence and D2 Receptor Availability in the Functional Subdivisions of the Striatum: Relationship with CocaineSeeking Behavior Neuropsychopharmacology 29: 1190-1202. 
Martinez D, Gil R, Slifstein M, Hwang DR, Huang Y, Perez A, Kegeles L, Talbot P, Evans S, Krystal J, Laruelle M, Abi-Dargham A. 2005. Alcohol Dependence Is Associated with Blunted Dopamine Transmission in the Ventral Striatum. Biological Psychiatry 58: 779 -786.

Martinez D, Orlowska D, Narendran R, Slifstein M, LiU F, Kumar D, Broft A Van Heertum R, Kleber HD. 2010. Dopamine type $2 / 3$ receptor availability in the striatum and social status in human volunteers. Biological psychiatry 67(3): 275-278.

Martinon F, Burns K, Tschopp J. 2002. The inflammasome: a molecular platform triggering activation of inflammatory caspases and processing of pro-IL-beta. Molecular cell 10(2): 417-426.

Matousek SB, Hein AM, Shaftel SS, Olschowka JA, Kyrkanides S, O'Banion MK. 2010. Cyclooxygenase-1 mediates prostaglandin E2 elevation and contextual memory impairment in a model of sustained hippocampal interleukin-1 $\beta$ expression. Journal of Neurochemistry 114(1): 247-258.

Matsuoka Y, Furuyashiki T, Yamada K, Nagai Y, Bito H, Tanaka Y, Kitaoka S, Ushikubi F, Nabeshima T, Narumiya S. 2005. Prostaglandin E receptor EP1 controls impulsive behavior under stress. PNAS 102(44): 16066-16071.

Matsuwaki T, Eskilsson A, Kugelberg U, Jönsson J, Blomqvist A. 2014. Interleukin-1 $\beta$ induced activation of the hypothalamus-pituitary-adrenal axis is dependent on interleukin-1 receptors on nonhematopoietic cells. Brain, behavior, and Immunity 40: 166-173.

McCann MJ, Verbalis JG, Stricker EM. 1989. LiCl and CCK inhibit gastric emptying and feeding and stimulate OT secretion in rats. The American Journal of Physiology 256: 463-468.

Merali Z, Brennan K, Brau P, Anisman H. 2003. Dissociating anorexia and anhedonia elicited by interleukin-1 $\beta$ : antidepressant and gender effects on responding for "free chow" and "earned" sucrose. Psychopharmacology 165: 413-418.

Millan MJ. 1990. K-opioid receptors and analgesia. Trends in Pharmacological Sciences 11(2): 70-76.

Minami T, Nakano H, Kobayashi T, Sugimoto Y, Ushikubi F, Ichikawa A, Narumiya S, Ito S. 2001. Characterization of EP receptor subtypes responsible for prostaglandin E2-induced pain responses by use of EP1 and EP3 receptor knockout mice. British Journal of Pharmacology 133(3): 438-444.

Molleman A. 2003. Patch Clamping. John Wiley and sons, LTD.

Morgan D, Grant KA, Gage HD, Mach RH, Kaplan JR, Prioleau O, Nader SH, Buchheimer N, Ehrenkaufer RL, Nader MA. 2002. Social dominance in monkeys: dopamine D2 receptors and cocaine self-administration. Nature Neuroscience 5: 169-174.

Mormede C, Castanon N, Medina C, Dantzer R. 2003. Conditioned place aversion with interleukin-1 $\beta$ in mice is not associated with activation of the cytokine network. Brain, Behavior, and Immunity 17(2): $110-120$.

Mul JD, Van Boxtel R, Bergen DJ, Brans MA, Brakkee JH, Toonen PW, Garner KM, Adan RA, Cuppen E. Melanocortin receptor 4 deficiency affects body weight regulation, grooming behavior, and substrate preference in the rat. Obesity 20(3): 612-621.

Murakami M, Naraba H, Tanioka T, Semmyo N, Nakatani Y, Kojima F, Ikeda T, Fueki M, Ueno A, Oh S, Kudo I. 2000. Regulation of prostaglandin E2 biosynthesis by inducible membrane-associated 
prostaglandin E2 synthase that acts in concert with cyclooxygenase-2. The Journal of Biological Chemistry 275(42): 32783-32792.

Myint AM, Bondy B, Baghai T, Eser D, Northdurfter C, Schüle C, Zill P, Müller N, Rupprecht R, Schwarz MJ (2013). Tryptophan metabolism and immunogenetics in major depression: A role for interferon- $\gamma$ gene. Brain, Behavior and Immunity 31: 128-133.

Ngampramuan S, Cerri M, Del Vecchio F, Corrigan JJ, Kamphee A, Dragic AS, Rudd JA, Romanovsky AA, Nalivaiko E. 2014. Thermoregulatory correlates of nausea in rats and musk shrews. Oncotarget 5(6): $1565-1575$.

Nguyen M, Camenisch T, Snouwaert JN, Hicks E, Coffman TM, Anderson PA, Malouf NN, Koller BH. 1997. The prostaglandin receptor EP4 triggers remodelling of the cardiovascular system at birth. Nature 390(6655):78-81.

Neurath MF. 2014. Cytokines in inflammatory bowel disease. Nature Reviews Immunology 14: 329342.

Nilsberth C, Elander L, Hamzic N, Norell M, Lönn J, Engström L, Blomqvist A. 2009. The role of Interleukin-6 in Lipopolysaccharide-induced fever by mechanisms independent of Prostaglandin E2. Neuroendocrinology 150(4): 1850-1860.

Nilsson A, Wilhelms DB, Mirrasekhian E, Jaarola M, Blomqvist A, Engblom D. 2017a. Inflammationinduced anorexia and fever are elicited by distinct prostaglandin dependent mechanisms, whereas conditioned taste aversion is prostaglandin independent. Brain, behavior, and Immunity 61: 236-243.

Nilsson A, Elander L, Hallbeck M, Kugelberg UÖ, Engblom D, Blomqvist A. 2017b. The involvement of prostaglandin E2 in interleukin-1 $\beta$ evoked anorexia is strain dependent. Brain, behavior, and Immunity 60: 27-31.

Nunes EJ, Randall PA, Estrada A, Epling B, Hart EE, Lee CA, Baqi Y, Müller CE, Correa M, Salamone JD. 2014. Effort-related motivational effects of the pro-inflammatory cytokine interleukin 1-beta: studies with the concurrent fixed ratio 5/chow feeding choice task. Psychopharmacology 231(4): 727736.

Oakman SA, Faris PL, Kerr PE, Cozzari C, Hartman BK. 1995. Distribution of Pontomesencephalic Cholinergic Neurons Projecting to Substantia Nigra Differs Significantly from Those Projecting to Ventral Tegmental Area. The Journal of Neuroscience 15(9): 5859-5869.

O'Connor JC, Lawson MA, Andre C, Briley EM, Szegedi SS, Lestage J, Castanon N, Herkenham M, Dantzer R, Kelley KW. 2009a. Induction of IDO by Bacille Calmette-Guerin is responsible for development of murine depressive-like behavior. The Journal of Immunology 182: 3202-3212.

O'Connor JC, André C, Wang Y, Lawson MA, Szegedi SS, Lestage J, Castanon N, Kelly KW, Dantzer R. 2009b. Interferon-gamma and tumor necrosis factor-alpha mediate the upregulation of indoleamine 2,3-dioxygenase and the induction of depressive-like behavior in mice in response to bacillus CalmetteGuerin. Journal of Neuroscience 29(13): 4200-4209.

O'Connor JC, Lawson MA, André C, Briley EM, Szegedi SS, Lestage J, Castanon N, Herkenham M, Dantzer R, Kelley KW. 2009c. Induction of IDO by Bacille Calmette-Guérin Is Responsible for Development of Murine Depressive-Like Behavior. Journal of Immunology 182(5): 3202-3212. 
O'Neil LAJ, Golenbock D and Bowie AG. 2013. The history of Toll-like receptors redefining innate immunity. Nature Reviews Immunology 13(6): 453-460.

Ophuis RJAO, Boender AJ, van Rozen AJ, Adan RAH. 2014. Cannabinoid, melanocortin and opioid receptor expression on DRD1 and DRD2 subpopulations in rat striatum. Frontiers in Neuroanatomy $8: 1-12$.

Oprica M, Zhu S, Goiny M, Pham TM, Mohammed AH, Winblad B, Bartfai T, Schultzberg M. 2005. Transgenic overexpression of interleukin-1 receptor antagonist in the CNS influences behavior, serum corticosterone and brain monoamines. Brain, Behavior, and Immunity 19: 223-234.

Ota H, Katanosaka K, Murase S, Furuyashiki T, Narumiya S, Mizumura K. 2017. EP2 receptor plays pivotal roles in generating mechanical hyperalgesia after lengthening contractions. Scandinavian Journal of Medicine \& Science in Sports doi: 10.1111/sms.12954 (ahead of print).

Panaro BL and Cone RD. 2013. Melanocortin-4 receptor mutations paradoxically reduce preference for palatable foods. PNAS 110(17): 7050-7055.

Park JY, Pillinger MH, Abramson SB. 2006. Prostaglandin E2 synthesis and secretion: the role of PGE2 synthases. Clinical Immunology 119(3): 229-240.

Pfeiffer A, Branti V, Herz A, Emrich HM. 1986. Psychotomimesis mediated by kappa opiate receptors. Science 233(4765): 774-776.

Pinto L, Goard MJ, Estandian D, Xu M, Kwan AC, Lee SH, Harrison TC, Feng G, Dan Y. 2013. Fast modulation of visual perception by basal forebrain cholinergic neurons. Nature Neuroscience 16(12): 1857-1863.

Platanias LC. 2005. Mechanisms of type-I- and type-II-interferon-mediated signaling. Nature Reviews Immunology 5(5): 375-386.

Pliakas AM, Carlson RR, Neve RL, Konradi C, Nestler EJ, Carlezon WA. 2001. Altered responsiveness to cocaine and increased immobility in the forced swim test associated with elevated CAMP response element-binding protein expression in nucleus accumbens. The Journal of Neuroscience 21(18): 73977403.

Qi J, Zhang S, Wang HL, Barker DJ, Miranda-Barrientos J, and Morales M. 2016. VTA glutamatergic inputs to nucleus accumbens drive aversion by acting on GABAergic interneurons. Nature Neuroscience 19(5):725-33.

Quan N, Whiteside M, Herkenham M. 1998. Time course and localization patterns of interleukin-1beta messenger RNA expression in brain and pituitary after peripheral administration of lipopolysaccharide. Neuroscience 83(1): 281-293.

Ramachandi VA, Umhau J, Pavon FJ, et al. 2011. A genetic determinant of the striatal dopamine response to alcohol in men. Molecular Psychiatry 16(8): 809-817.

Re F, Sironi M, Matteucci C, Introna M, Orlando S, Penton-Rol G, Dower SK, Sims JE, Colotta F, Mantovani A. 1996. Inhibition of interleukin-1 responsiveness by type II gene transfer: a surface receptor with anti- interleukin-1 function. The Journal of Experimental Medicine 183(4): 1841-1850.

Reichenberg A, Yirmiya R, Schuld A, Kraus T, Haack M, Morag A, Pollmächer T. 2001. Cytokineassociated emotional and cognitive disturbances in humans. Archieves of General Psychiatry 58(5): 445-452. 
Reimers J, Wogensen LD, Welinder B, Hajnaes KR, Poulsen SS, Nilsson P, Nerup J. 1991. The pharmacokinetics, distribution, and degradation of human recombinant interleukin 1 beta in normal rats. Scandinavian Journal of Immunology 34(5): 597-610.

Robinson TE, Berridge KC. 2001. Incentive-sensitization and addiction. Addiction 96(1): 103-114.

Robinson TE, Berridge KC. 2003. Addiction. Annual Reviews of Psychology 54: 25-53.

Rossi S, Studer V, Motta C, Polidoro S, Perugini J, Macchiarulo G, Giovannetti AM, Pareja-Gutierrez L, Calo A, Colonna I, Furlan R, Martino G, Centonze D. 2017. Neuroinflammation drives anxiety and depression in relapsing-remitting multiple sclerosis. Neurology 89(13): 1338-1347.

Roth BL. 2016. DREADDs for neuroscientists. Neuron 89: 683-694.

Saha A, Engström L, Mackerlova L, Jakobsson P, Bomqvist A. 2005. Impaired febrile response to immune challenge in mice deficient in microsomal prostaglandin E synthase-1. Am J Physiol Regul Integr Comp Physiol 288: 1100-1107.

Salamone JD, Cousins MS, Bucher S. 1994. Anhedonia or anergia? Effects of haloperidol and nucleus accumbens dopamine depletion on instrumental response selection in a T-maze cost/benefit procedure. Behavioural brain research 65(2): 221-229.

Sampson TR, Debelius JW, Thron T, Janssen S, Shastri GG, Ilhan ZE, Challis C, et al. 2016. Gut Microbiota Regulate Motor Deficits and Neuroinflammation in a Model of Parkinson's Disease. Cell 167(6): 1469-1480.

Sanchis-Segura C and Spanagel R. 2006. Behavioural assessment of drug reinforcement and addictive features in rodents: and overview. Addiction Biology 11(1): 2-38.

Sandman CA, Kastin AJ. 1981. Intraventricular administration of MSH induces hyperalgesia in rats. Peptides 2(2): 231-233.

Saper CB, Romanovsky AA, Scammel TE. 2012. Neural circuitry engaged by prostaglandins during the sickness syndrome. Nature Neuroscience 15(8): 1088-1095.

Saper CB and Sawchenko PE. 2003. Magic peptides, magic antibodies: Guidelines for appropriate controls for immunohistochemistry. The Journal of Comparative Neurology 465: 161-163.

Schett G, Dayer JM and Manger B. 2016. Interleukin-1 function and role in rheumatic disease. Nature Reviews Rheumatology 12: 14-24.

Schmidt LS, Thomsen M, Weikop P, Dencker D, Wess J, Woldbye DPD, Wortwein G, Fink-Jensen A. 2011. Increased cocaine self-administration in M4 muscarinic acetylcholine receptor knockout mice. Psychopharmacology 216: 367-378.

Schnütgen F, Doerflinger N, Calleja C, Wendling O, Chambon P, Ghyselinck NB. 2003. A directional strategy for monitoring Cre-mediated recombination at the cellular level in the mouse. Nature Biotechnology 21(5): 562-565.

Schratzberger P, Dunzendorfer S, Reinisch N, Kähler CM, Herold M, Wiedermann CJ. 1998. Release of chemoattractants for human monocytes from endothelial cells by interaction with neutrophils. Cardiovascular Research 38(2): 516-521. 
Schroeder HW. 2015. The evolution and development of the antibody repertoire. Frontiers in Immunology 6: 33.

Schwab JM, Brechtel K, Nguyen TD, Schluesener HJ. 2000. Persistent accumulation of cyclooxygenase-1 (COX-1) expressing microglia/macrophages and upregulation by endothelium following spinal cord injury. Journal of Neuroimmunology 111(2): 122-130.

Shukuri M, Takashima-Hirano M, Tokuda K, Takashima T, Matsumura K, Inoue O, Doi H, Suzuki M, Watanabe Y, Onoe H. 2011. In vivo expression of cyclooxygenase-1 in activated microglia and macrophages during neuroinflammation visualized by PET with $11 \mathrm{C}$-ketoprofen methyl ester. Journal of Nuclear Medicine 52(7): 1094-1101.

Schultz W and Romo R. 1990. Dopamine neurons of the monkey midbrain: contingencies of responses to stimuli eliciting immediate behavioral reactions. Journal of Neurophysiology 63(3): 607-624.

Shultz W, Dayan P, Montague PR. 1997. A neural substrate of prediction and reward. Science 272(5306): 1593-1599.

Shultz W. 2013. Updating dopamine reward signals. Current opinion in neurobiology 23(2): 229-238.

Setiawan E, Wilson AA, Mizrahi R, Rusjan PM, Miler L, Rajkowska G, Suridjan I, Kennedy JL, Rekkas PV, Houle S, Meyer JH. 2015. Role of translocator protein density, a marker of neuroinflammation, in the brain during major depressive episodes. JAMA Psychiatry 72(3): 268-275.

Silverman MN, Macdougall MG, Hu F, Pace TW, Raison CL, Miller AH. 2007. Endogenous glucocorticoids protect against TNF-alpha-induced increases in anxiety-like behavior in virally infected mice. Molecular Psychiatry 12(4): 408-417.

Simen BB, Duman CH, Simen AA, Duman RS. 2006. TNFalpha signaling in depression and anxiety: behavioral consequences of individual receptor targeting. Biological Psychiatry 59(9): 775-785.

Singh AK, Zajdel J, Mirrasekhian E, Almoosawi N, Frisch I, Klawonn AM, Jaarola M, Fritz M, Engblom D. 2017. Prostaglandin-mediated inhibition of serotonin signaling controls the affective component of inflammatory pain. The Journal of Clinical Investigation 127(4): 1370-1374.

Sokolova ES, Lyudyno VI, Simbirtsev AS, Klimenko VM. 2007. The psychomodulatory actin of subpyrogenic doses of interleukin- $1 \beta$ in conditions of chronic administration to rats. Neuroscience and Behavioral Physiology 37(5): 499-504.

Solomon RL. 1980. The opponent-process theory of acquired motivation: the costs of pleasure and the benefits of pain. The American psychologist 35(8): 691-712.

Spiteri T, Le Pape G, Ågmo A. 2000. What is learned during place preference conditioning? A comparison of food-and morphine-induced reward. Psychobiology 28(3): 367-382.

Steketee JD and Kalivas PW (2011). Drug Wanting: Behavioral Sensitization and Relapse to DrugSeeking Behavior. Pharmacological Reviews 63(2): 348-365.

Sternberg N, Sauer B, Hoess R, Abremski K. 1986. Bacteriophage P1 cre gene and its regulatory region. Evidence for multiple promoters and for regulation by DNA methylation. Journal of molecular biology 187(2): 197-212.

Sternberger LA. 1979. Immunocytochemistry. John Wiley and sons Inc. 
Stuber GD, Roitman MF, Phillips PE, Carelli RM, Wightman RM. 2005. Rapid dopamine signaling in the nucleus accumbens during contingent and noncontingent cocaine administration. Neuropsychopharmacology 30: 853-863.

Stuber GD, Sparta DR, Stamatakis AM, van Leeuwen WA, Hardjoprajitno JE, Cho S, Tye KM, Kempadoo KA, Zhang F, Deisseroth $\mathrm{K}$ et al. 2011. Excitatory transmission from the amygdala to nucleus accumbens facilitates reward seeking. Nature 475(7356): 377-80.

Sun D, Ding A. 2006. MyD88-mediated stabilization of interferon-gamma-induced cytokine and chemokine mRNA. Nature Immunology 7(4): 375-381.

Takahashi Y, Schoenbaum G, Niv Y. 2008. Silencing the critics: understanding the effects of cocaine sensitization on dorsolateral and ventral striatum in the context of an actor/critic model. Frontiers in Neuroscience 2(1): 86-99.

Tanaka Y, Furuyashiki T, Momiyama T, Namba H, Mizoguchi A, Mitsumori T, Kayahara T, Shichi H, Kimura K, Matsuoka T, Nawa H, Narumiya S. 2009. Prostaglandin E receptor EP1 enhances GABAmediated inhibition of dopaminergic neurons in the substantia nigra pars compacta and regulates dopamine level in the dorsal striatum. European Journal of Neuroscience 30(12): 2338-2346.

Tanaka K, Furuyashiki T, Kitaoka S, Senzai Y, Imoto Y, Segi-Nishida E, Deguchi Y, Breyer RM, Breyer MD, Narumiya S. 2012. Prostaglandin E2-Mediated Attenuation of Mesocortical Dopaminergic Pathway Is Critical for Susceptibility to Repeated Social Defeat Stress in Mice. The Journal of Neuroscience 32(12); 4319-4329.

Tazi A, Dantzer R, Crestani F, Le Moal M. 1988. Interleukin-1 induces conditioned taste aversion in rats: a possible explanation for its pituitary-adrenal stimulating activity. Brain Research 473(2): 369371.

Teeling JL, Cunningham C, Newman TA, Perry VH. 2010. The effect of non-steroidal antiinflammatory agents on behavioural changes and cytokine production following systemic inflammation: Implications for role of COX-1. Brain, Behaviour and Immunity 2010: 409-419.

Terraneo A, Leggio L, Saladini M, Ermani M, Bonci A, Gallimberti L. 2016. Transcranial magnetic stimulation of dorsolateral prefrontal cortex reduces cocaine use: A pilot study. European Neuropsychopharmacology 26(1): 37-44.

Thiele TE, van Dijk G, Yagaloff KA, Fisher SL, Schwartz M, Burn P, Seeley RJ. 1998. Central infusion of melanocortin agonist MTII in rats: assessment of c-fos expression and taste aversion. The American Journal of Physiology 274: 248-254.

Thomas KR and Capecchi. 1987. Site-directed mutagenesis by gene targeting in mouse embryo-derived stem cells. Cell 51(3): 503-512.

Thompson S, Clarke AR, Pow AM, Hooper ML, Melton DW. 1989. Germ line transmission and expression of a corrected HPRT gene produced by gene targeting in embryonic stem cells. Cell 56(2): 313-321.

Thornberry NA, Bull HG, Calaycay JR, Chapman KT, Howard AD et al. 1992. A novel heterodimeric cysteine protease is required for interleukin-1 $\beta$ processing in monocytes. Nature 356(6372): 768-774.

Turgeon B and Meloche S. 2009. Interpreting neonatal lethal phenotypes in mouse mutants: insights into gene function and human diseases. Physiological reviews 89(1): 1-26. 
Tye KM and Deisseroth K. 2012. Optogenetic investigation of neural circuits underlying brain disease in animal models. Nature Reviews Neuroscience 13: 251-266.

Tzavara E, Bymaster FP, Davis RJ, Wade MR, Perry KW, Wess J, McKinzie DL, Felder C, Nomikos GG. 2004. M4 muscarinic receptors regulate the dynamics of cholinergic and dopaminergic neurotransmission: relevance to the pathophysiology and treatment of related central nervous system pathologies. The FASEB Journal 18(12):1410-1412.

Tzschentke TM. 2007. Measuring reward with the conditioned place preference (CPP) paradigm: Update of the last decade. Addiction Biology 12(4): 227-462.

Ungerstedt U. 1971. Adipsia and aphagia after 6-Hydroxydopamine induced degeneration of the Nigrostriatal Dopamine system. Acta Physiologica 82(367): 95-122.

Ungless MA, Whistler JL, Malenka RC, Bonci A. 2001. Single cocaine exposure in vivo induces longterm potentiation in dopamine neurons. Nature 411(6837): 583-587.

Urban DJ and Roth BL. 2015. DREADDs (designer receptors exclusively activated by designer drugs): Chemogenetic tools with therapeutic utility. Annual review of pharmacology and toxicology 55: 399417.

Ushikubi F, Segi E, Sugimoto Y, Murata T, Matsuoka T, Kobayashi T, Hizaki H, Tuboi K, Katsuyama M, Ichikawa A, Tanaka T, Yoshida N, Narumiya S. 1998. Impaired febrile response in mice lacking the prostaglandin E receptor subtype EP3. Nature 395(6699): 281-284.

Van Zee KJ, Kohno T, Fischer E, Rock CS, Moldawer LL, Lowry SF. 1992. Tumor necrosis factor soluble receptors circulate during experimental and clinical inflammation and can protect against excessive tumor necrosis factor alpha in vitro and in vivo. PNAS 89(11): 4845-4849.

Volkow ND, Fowler JS, Wang GJ, Swanson JM, Telang F. 2007. Dopamine in drug abuse and addiction: results of imaging studies and reatment implications. Archieves of Neurology 64(11): 15751579.

Von Bernuth H, Picard C, Jin Z, Pankla R, Xiao H, Charabieh M et al. 2008. Pyogenic bacterial infections in humans with Myd88 deficiency. Science 321(5889): 691-696.

Vongs A, Lynn NM, Rosenblum CI. 2004. Activation of MAP kinase by MC4-R through PI3 kinase. Regulatory peptides 120: 113-118.

Wakonigg G, Sturm K, Saria A, Zernig G. 2003. Opioids, cocaine, and food change runtime distribution in a rat runway procedure. Psychopharmacology 169: 52-59.

Wang D, Xiaobing H, Zhao Z, Feng Q, Lin R, Sun Y, Ding T, Xu F, Luo M, Zhan C. Whole-brain mapping of the direct inputs and axonal projections of POMC and AgRP neurons. Frontiers in Neuroanatomy 9: 1-17.

Wajant H, Pfizenmaier K, Scheurich P. 2003. Tumor necrosis factor signaling. Cell Death Differ. 10(1): 45-65.

Waterston RH, Lindblad-Toh K, Birney E et al. 2002. Initial sequencing and comparative analysis of the muse genome. Nature 420(6915): 520-562.

Wess J, Eglen RM, Gautam D. 2007. Muscarinic acetylcholine receptors: mutant mice provide new insights for drug development. Nature reviews 6: 721-733. 
Wilhelms DB, Kirilov M, Mirrasekhian E, Eskilsson A, Kugelberg UÖ, Klar C, Ridder DA, Herschman HR, Schwaninger M, Blomqvist A, Engblom D. 2014. Deletion of prostaglandin E2 synthesizing enzymes in brain endothelial cells attenuates inflammatory fever. The Journal of Neuroscience 34(35): 11684-11690.

Wilson KP, Black JA, Thomson JA, Kim EE, Griffith JP, Navia MA, Murcko MA, Chambers SP, Aldape RA, Raybuck SA, Livingston DJ. 1994. Structure and mechanism of interleukin-1 $\beta$ converting enzyme. Nature 370(6487): 270-275.

Witten IB, Lin S, Brodsky M, Prakash R, Diester I, Anikeeva P, Gradinaru V, Ramakrishnan C and Deisseroth K. 2010. Cholinergic Interneurons Control Local Circuit Activity and Cocaine Conditioning. Science 330(6011): 1677-1681.

Wohleb ES, Patterson JM, Sharma V, Quan N, Godbout JP, Sheridan JF. 2014. Knockdown of interleukin-1 receptor type-1 on endothelial cells attenuated stress-induced neuroinflammation and prevented anxiety-like behavior. The Journal of Neuroscience 34(7): 2583-2591.

Yu Z, Fukushima H, Ono C, Sakai M, Kasahara Y, Kikuchi Y, Gunawansa N, Takahashi Y, Matsuoka H, Kida S, Tomita H. 2017. Microglial production of TNF-alpha is a key element of sustained fear memory. Brain, Behavior and Immunity 59: 313-321.

Zant JC, Kim T, Prokai L, Szarka S, McNally J, McKenna JT, Shukla C, Yang C, Kalinchuk AV, McCarley RW, Brown RE, Basheer R. Cholinergic neurons in the basal forebrain promote wakefulness by actions on neighboring non-cholinergic neurons: An opto-dialysis study. The Journal of Neuroscience 36(6): 2057-2067.

Zhan C, Zhou J, Feng Q, Zhang JE, Lin S, Bao J, Wu P, Luo M. 2013. Acute and long-term suppression of feeding behavior by POMC neurons in the brainstem and hypothalamus, respectively. The Journal of Neuroscience 33(8): 3624-3632.

Zhang J, Rivest S. 1999. Distribution, regulation and colocalization of the genes encoding the EP2- and EP4-PGE2 receptors in the rat brain and neuronal responses to systemic inflammation. European Journal of Neuroscience 11(8): 2651-2668.

Zhang W, Basile AS, Gomeza J, Volpicelli LA, Levey AI, Wess J. 2002. Characterization of Central Inhibitory Muscarinic Autoreceptors by the Use of Muscarinic Acetylcholine Receptor Knock-Out Mice. The Journal of Neuroscience 22(5): 1709-1717.

Zhu Y, Wienecke CF, Nachtrab G, and Chen X. 2016. A thalamic input to the nucleus accumbens mediates opiate dependence. Nature 530(7589): 219-22.

Zijlstra M, Li E, Sajjadi F, Subramani S, Jaenisch R. 1989. Germ-line transmission of a disrupted beta 2-microglobulin gene produced by homologous recombination in embryonic stem cells. Nature 342(6248): 435-438.

Zincarelli C, Soltys S, Rengo G, Rabinowitz JE. 2008. Analysis of AAV Serotypes 1-9 Mediated Gene Expression and Tropism in Mice After Systemic Injection. Molecular Therapy 16(6): 1073-1080. 


\section{Papers}

The papers associated with this thesis have been removed for copyright reasons. For more details about these see:

http:// urn.kb.se/ resolve?urn=urn:nbn:se:liu:diva-143459 